DESY 18-211, HU-EP-18/38

\title{
The Hierarchy Problem and the Cosmological Constant Problem Revisited
}

\author{
Higgs inflation and a new view on the SM of particle physics \\ Fred Jegerlehner \\ Humboldt-Universität zu Berlin, Institut für Physik, \\ Newtonstrasse 15, D-12489 Berlin, Germany \\ Deutsches Elektronen-Synchrotron (DESY), \\ Platanenallee 6, D-15738 Zeuthen, Germany \\ e-mail: fjeger@physik.hu-berlin.de
}

May 7, 2019

\begin{abstract}
We argue that the Standard Model (SM) in the Higgs phase does not suffer from a "hierarchy problem" and that similarly the "cosmological constant problem" resolves itself if we understand the SM as a low energy effective theory (LEET) emerging from a cutoff-medium at the Planck scale. We actually take serious Veltman's "The Infrared - Ultraviolet Connection" addressing the issue of quadratic divergences and the related huge radiative correction predicted by the SM in the relationship between the bare and the renormalized theory, usually called "the hierarchy problem" and claimed that this has to be cured. We discuss these issues under the condition of a stable Higgs vacuum, which allows extending the SM up to the Planck cutoff. The bare Higgs boson mass then changes sign below the Planck scale, such that the SM in the early universe is in the symmetric phase. The cutoff enhanced Higgs mass term, as well as the quartically enhanced cosmological constant term, provide a large positive dark energy that triggers the inflation of the early universe. Reheating follows via the decays of the four unstable heavy Higgs particles, predominantly into top-antitop pairs, which at this stage are massless. Preheating is suppressed in SM inflation since in the symmetric phase bosonic decay channels are absent at tree level. The coefficients of the shift between bare and renormalized Higgs mass as well as of the shift between bare and renormalized vacuum energy density exhibit close-by zeros at about $7.7 \times 10^{14} \mathrm{GeV}$ and $3.1 \times 10^{15} \mathrm{GeV}$, respectively. The zero of the Higgs mass counter term triggers the electroweak phase transition, from the low energy Higgs phase and to the symmetric phase above the transition point. Since inflation tunes the total energy density
\end{abstract}


to take the critical value of a flat universe and all contributing components are positive, it is obvious that the cosmological constant today is naturally a substantial fraction of the total critical density. Thus taking cutoff enhanced corrections seriously the Higgs system provides besides the masses of particles in the Higgs phase also dark energy, inflation and reheating in the early universe. The main unsolved problem in our context remains the origin of dark matter. Higgs inflation is possible and likely even unavoidable provided new physics does not disturb the known relevant SM properties substantially. The scenario highly favors understanding the $\mathrm{SM}$ and its main properties as a natural structure emerging at long distance. This in particular concerns the SM symmetry patterns and their consequences.

Keywords: Higgs vacuum stability hierarchy problem cosmological constant problem inflation

PACS $14.80 . \mathrm{Bn} \cdot 11.10 . \mathrm{Gh} \cdot 12.15 . \mathrm{Lk} \cdot 98.80 . \mathrm{Cq}$

Invited talk at the Workshop "Naturalness, Hierarchy and Fine Tuning" RWTH Aachen, 28 February 2018 to 2 March 2018, Aachen,Germany 


\section{Contents}

1 Prelude: Higgs inflation in a nutshell 2

2 The Higgs boson discovery - the SM completion 7

2.1 Matching conditions: $\overline{\mathrm{MS}}$ parameters in terms of physical parameters 9

2.2 The SM running parameters . . . . . . . . . . . . . 13

3 Thoughts on guiding principles and paradigms in particle physics 15

4 The Hierarchy Problem revisited $\quad 21$

5 Running SM parameters trigger the Higgs mechanism 25

5.1 Vacuum stability and effective potential . . . . . . . . . . 29

6 The cosmological constant - dark energy provided by the Higgs scalar 34

6.1 A self-organized cosmological constant? . . . . . . . . . . 36

7 Inflation and reheating $\quad 38$

7.1 Reheating by Higgs boson decays . . . . . . . . . . . . . . . 39

8 Remark on trans-Planckian Higgs fields 40

9 Remarks for the skeptic $\quad 42$

10 Summary and conclusions $\quad 43$

11 Appendix: How natural is the minimal SM? 46

\section{Prelude: Higgs inflation in a nutshell}

In order to give a quick overview of what will be the essential conclusion of the analysis I start with this prelude (see [1,2]). The Standard Model (SM) hierarchy problem [3] is well known and addressed very frequently to motivate Beyond the Standard Model (BSM) scenarios in general and a supersymmetric extension of the SM in particular. The renormalized Higgs boson mass is small, at the ElectroWeak (EW) scale, the bare one is huge due to radiative corrections growing quadratically with the ultraviolet (UV) cutoff, which is assumed to be given by the Planck scale $\Lambda_{\mathrm{Pl}} \sim 10^{19} \mathrm{GeV}^{1}$. The cutoff

\footnotetext{
${ }^{1}$ The Planck medium, which we may call ether, somehow gets shaped by gravity and quasi-particle interactions emergent in the SM at low energies. It is characterized by the well known fundamental Planck cutoff $\Lambda_{\mathrm{Pl}}$ or equivalently the Planck mass $M_{\mathrm{Pl}}$, which derive from the basic fundamental constants, the speed of light $c$ characterizing special relativity, the Planck constant $\hbar$ intrinsic to quantum physics and Newton's constant $G_{N}$ the dimensionful key parameter of gravity. Unified they provide an intrinsic length $\ell_{\mathrm{Pl}}$, the Planck length, which also translates into the Planck time $t_{\mathrm{Pl}}$ and the Planck temperature $T_{\mathrm{Pl}}$ :

Planck length: $\ell_{\mathrm{Pl}}=\sqrt{\frac{\hbar G_{N}}{c^{3}}}=1.616252(81) \times 10^{-33} \mathrm{~cm}$,

Planck time: $t_{\mathrm{Pl}}=\ell_{\mathrm{Pl}} / c=5.4 \times 10^{-44} \mathrm{sec}$,

Planck (energy) scale: $M_{\mathrm{Pl}}=\sqrt{\frac{c \hbar}{G_{N}}}=1.22 \times 10^{19} \mathrm{GeV}$,
} 


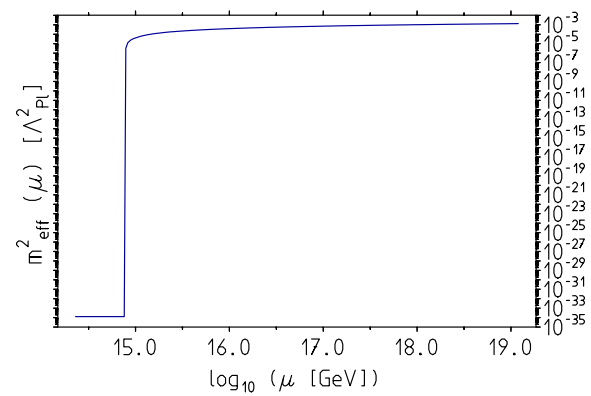

Figure 1: The square of the effective Higgs-field mass as a function of the log of the scale $\mu$, in units of $\Lambda_{\mathrm{Pl}}^{2}$. The effective mass is given by the bare mass at short distances and by the renormalized one at low energy.

dependence is illustrated in Fig. 1 assuming the cutoff as a renormalization scale and the SM Renormalization Group (RG) ruling the scale dependence of the SM couplings (see below). It is the RG improved version of Veltman's "The Infrared - Ultraviolet Connection" [4], where the SM renormalization of the Higgs boson mass ( $m_{0}$ the bare, $m$ the renormalized mass)

$$
m_{0}^{2}=m^{2}+\delta m^{2} ; \delta m^{2}=\frac{\Lambda_{\mathrm{Pl}}^{2}}{\left(16 \pi^{2}\right)} C(\mu),
$$

has been addressed (see also [5-8]). The coefficient function $C(\mu)$ depends on the dimensionless SM couplings, which depend on the renormalization scale logarithmically only. For an early discussion of the impact of running couplings to the Higgs mass term and the problem of fine-tuning see [9]. The Higgs mass counterterm is huge when we adopt the Planck scale as the cutoff to regulate UV singularities. Is this a problem? Is this unnatural? In the first instance, it is a prediction of the SM! At low energy, we see what we see (what is to be seen): the renormalizable, renormalized SM [10-12] as it describes close to all we know up to LHC energies. But what does the SM look like if we go to very high energies even to the Planck scale? Not too far below the Planck-scale, we start to see the bare theory i.e. the SM with its bare short distance effective parameters, so in particular a very heavy Higgs boson, which likely is moving at most very slowly. The potential energy

$$
V(\phi)=\frac{m^{2}}{2} \phi^{2}+\frac{\lambda}{24} \phi^{2}
$$

then is large, while the kinetic energy $\frac{1}{2} \dot{\phi}^{2}$ is small, as a dedicated calculation shows. Here we have in mind the cosmological solutions of Einstein's General Relativity The-

Planck temperature: $\frac{M_{\mathrm{P}} c^{2}}{k_{\mathrm{B}}}=\sqrt{\frac{\hbar c^{5}}{G_{N} k_{\mathrm{B}}^{2}}}=1.416786(71) \times 10^{32^{\circ} \mathrm{K}}$.

In our context, they define a shortest distance $\ell_{\mathrm{Pl}}$ and a beginning of time $t_{\mathrm{Pl}}$. i.e. $t>t_{\mathrm{Pl}}$. The Planck era energy scale equivalently is set by $E_{\mathrm{Pl}}=\Lambda_{\mathrm{Pl}} \equiv M_{\mathrm{Pl}}$ or temperature $T_{\mathrm{Pl}}$, as for most time in the evolution of the early universe, when elementary particle physics is at work and before the epoch of formation of hadrons, particle processes are in thermal equilibrium, with well-known exceptions during inflation and the electroweak phase-transition. 
ory (GRT) for an isotropic universe of constant spatial curvature, parametrized by the Robertson-Walker metric ${ }^{2}$. The field $\phi$ is then a function of the cosmic time $t$ only and $\dot{\phi}$ is the corresponding time derivative. The Higgs boson contributes to the energymomentum tensor by providing the pressure $p=\frac{1}{2} \dot{\phi}^{2}-V(\phi)$ and the energy density $\rho=\frac{1}{2} \dot{\phi}^{2}+V(\phi)$. As we approach the Planck scale (bare theory) the slow-roll condition $\frac{1}{2} \dot{\phi}^{2} \ll V(\phi)$ is satisfied during some window in the time evolution and then $p \approx-V(\phi) ; \rho \approx+V(\phi)$ implies $p \approx-\rho$, which closely approximates the equation of state $p_{\Lambda}=-\rho_{\Lambda}$ of Dark Energy (DE) $\rho=\rho_{\Lambda}$, the equivalent of a Cosmological Constant (CC) $\Lambda$. DE follows a very special equation of state, only observed indirectly through Cosmic Microwave Background (CMB) [13-17] pattern and through Super Novae (SN) counts $[18,19]$. No lab system observation so far has been reported to my knowledge, although statistical mechanics system like the Ising model obey such a ground state equation (see e.g. [20]). Thus, as a consequence of the hierarchy boost, the SM Higgs boson in the early universe delivers a huge dark energy that is inflating the universe and, which mimics strong anti-gravity at work. The Friedmann equations together with energy conservation read

$$
\left(\frac{\dot{a}}{a}\right)^{2}+k / a^{2}=\frac{8}{3} \pi G_{N} \rho ; \quad \frac{\ddot{a}}{a}=-\frac{4}{3} \pi G_{N}(3 p+\rho) ; \dot{\rho}=-3 \frac{\dot{a}}{a}(\rho+p)
$$

and indeed if DE dominates we have $(3 p+\rho) \approx-2 \rho$ such that we have an accelerated expansion $\ddot{a} / a>0$ and $\frac{\mathrm{d} a}{a}=H(t) \mathrm{d} t$ which implies an exponential growth $a(t)=$ $\exp H t$ of the radius $a(t)$ of the universe. $H(t)=\dot{a}(t) / a(t)$ is the Hubble constant where $H \propto \sqrt{V(\phi)}$ in a DE dominated era. Inflation stops quite quickly as the field decays exponentially. The field equation

$$
\ddot{\phi}+3 H \dot{\phi} \simeq-V^{\prime}(\phi),
$$

for a potential dominated by the mass term $V(\phi) \approx \frac{m^{2}}{2} \phi^{2}$ represents a harmonic oscillator with friction and leads to Gaussian inflation as established by an analysis of the CMB pattern by the Planck mission [21]. One of the reasons why the inflation phenomenon must have happened in the early universe is that the universe looks flat today, while a flat universe in the absence of DE is exponentially unstable in its time evolution. This because then formally the strong energy condition $\rho+3 p \geq 0$ holds, which implies $\ddot{a} / a \leq 0$. So, different types of solutions of the Friedmann equations at $\rho_{\Lambda}=0$ deviate

\footnotetext{
${ }^{2}$ Einstein's field equation for the metric tensor $g_{\mu \nu}$, which incorporates the gravitational field, is given by $G_{\mu \nu}=\kappa T_{\mu \nu}$ where $\kappa=\frac{8 \pi G_{N}}{c^{2}}$ is the effective interaction constant, $G_{\mu \nu}=R_{\mu \nu}-\frac{1}{2} R g_{\mu \nu}$ is the Einstein curvature tensor (geometry) and $\stackrel{c}{T}_{\mu \nu}$ is the energy-momentum tensor (matter and radiation). Cosmology is shaped by Einstein gravity, which together with Weyl's postulate, that radiation and matter (galaxies etc.) on the cosmological scale behave like an ideal fluid, and the cosmological principle, assuming isotropy of space (implying homogeneity), fixes the form of the metric and of the energy-momentum tensor: 1) the metric (3-spaces of constant curvature $k= \pm 1,0)$ takes the form $\mathrm{d} s^{2}=(c \mathrm{~d} t)^{2}-a^{2}(t)\left[\mathrm{d} r^{2} /\left(1-k r^{2}\right)+r^{2} \mathrm{~d} \Omega^{2}\right]$, where, in the comoving frame $\mathrm{d} s=c \mathrm{~d} t$ with $t$ the cosmic time; 2) the energy-momentum tensor takes the form $T^{\mu v}=(\rho(t)+p(t))(t) u^{\mu} u^{v}-p(t) g^{\mu v} ; u^{\mu} \doteq \frac{\mathrm{d} x^{\mu}}{\mathrm{d} s}$ where $\rho(t)$ is the density and $p(t)$ the pressure of the fluid. As a differential equation for the geometry factor $a(t)$ one obtains Friedmann's equations (3). One needs $\rho(t)$ and $p(t)$ (which are related by an equation of state characterizing the medium) in order to get the radius of the universe $a(t)$ and its evolution in time. The Higgs potential contributes $T_{\mu \nu}=\Theta_{\mu \nu}=V(\phi) g_{\mu \nu}+$ derivative terms, where $\Theta_{\mu v}$ is the symmetric energy-momentum tensor of the SM (or extensions of it). Only a scalar potential can contribute a term proportional to $g_{\mu \nu}$, which mimics a cosmological constant.
} 
dramatically during the 13.8 billion years life of the universe after the Big Bang. The "flattenization" by inflation is evident as the curvature term $k / a^{2}(t) \sim k \exp (-2 H t) \rightarrow 0$ drops exponentially independent of the curvature type. The latter, characterized by the normalized curvature $k=0, \pm 1$, distinguishes flat infinite, spherical closed or hyperbolic open geometries. It is very important to note that the $\mathrm{CC}$ given by the Higgs potential $V(\phi)$ in the symmetric phase is positive in any case, very different from the (much smaller) contribution from the Higgs-field vacuum expectation value (VEV) in the broken phase, which is negative [22]. This already shows that dynamical effects and in particular possible phase transitions make the CC depend on time, although it does not depend explicitly on $a(t)$, i.e., $\rho_{\Lambda} \propto a(t)^{0}$. As we know the matter density scales like $\rho_{m} \propto a(t)^{-3}$ while the radiation density decreases like $\rho_{\gamma} \propto a(t)^{-4}$ since radiation gets red-shifted in addition to its spatial dilution ${ }^{3}$.

Inflation tunes the total energy density to be that of a flat space (as if $k=0$ ), which according to (3) for $k=0$ requires a specific "critical" energy density

$$
\rho_{0, \text { crit }}=3 H_{0} /\left(8 \pi G_{N}\right)=\mu_{\text {crit }}^{4} \text { where } \mu_{\text {crit }} \approx 0.00247 \mathrm{eV} .
$$

With $H_{0}$ the present Hubble constant $\rho_{0 \text {,crit }}$ is the present total energy density. For an arbitrary mixture of dark energy, matter and radiation the first of the equations (3) reads

$$
\rho=\rho_{0, \text { crit }}\left[\Omega_{\Lambda}+\Omega_{m}\left(\frac{a_{0}}{a}\right)^{3}+\Omega_{\gamma}\left(\frac{a_{0}}{a}\right)^{4}\right],
$$

where $\Omega_{i}=\rho_{0, i} / \rho_{0 \text {,crit }}$ are the present fractional densities and $a_{0}=a\left(t_{0}\right)$ the spatial metric scale factor at present time $t_{0}$. Including the curvature term $\Omega_{k}=-k /\left(a_{0}^{2} H_{0}^{2}\right)$ we have

$$
\Omega_{\Lambda}+\Omega_{m}+\Omega_{\gamma}+\Omega_{k}=1
$$

as an exact equation, and when the curvature term is exponentially suppressed we very accurately have

$$
\Omega_{\Lambda}+\Omega_{m}+\Omega_{\gamma} \cong 1
$$

which is supported strongly by observation (CMB). Whatever constitutes the universe, the curvature constant is $k=+1, k=0$ or $k=-1$ according to whether the present density $\rho_{0}$ is greater than, equal to, or less than $\rho_{0, \text { crit }}$. A higher density $\rho_{0}>\rho_{0 \text {,crit }}$ implies a re-contraction of the initially (at the Big Bang) expanding universe, a lower density $\rho_{0}<\rho_{0, \text { crit }}$ would not be able to stop the expansion forever.

We know that $\rho_{0 \Lambda}=\mu_{0 \Lambda}^{4}$ today is about $\mu_{0 \Lambda}=0.00171 \mathrm{eV}$ which in a flat universe must be a fraction of the critical density, and actually has been determined to amount to $69.2 \pm 1.2 \%$. Since the non-DE components drop with a power of the radius $a(t)$ as

\footnotetext{
${ }^{3}$ Matter here includes dark matter (DM) and normal baryonic-matter (BM), the non-relativistic stuff; radiation includes all relativistic degrees of freedom: photons, neutrinos and at high energies other SM particles besides the Higgs bosons, which get boosted to be heavy because of their missing naturalness. Note that normal baryonic matter only emerges after the QCD [12] hadronization phase-transition,i.e. after protons and neutrons have been formed. In contrast, cold dark matter looks must have existed much earlier not too long after Planck time.
} 
time goes on, the asymptotic behavior is determined by $\rho_{\Lambda}$ solely. Friedmann solutions in GRT with non-vanishing cosmological constant have been discussed in [23,24].

We note that the large positive cosmological constant provided by the SM Higgs sector, on the one hand, effectuates the inflation that is needed to tune the total energy density into the critical density of flat space. This tells us that on the other hand, the cosmological constant has to be some fraction of the critical density, i.e. it is self-tuned to be small. So also the cosmological constant problem may turn out to get its natural explanation (see below).

Since inflation is strongly supported to have happened by observation, we must assume the existence of an appropriate scalar field, and the Higgs field is precisely such a field we need and within the SM it has the properties that promote it to be the inflaton field. In contrast to other inflation models, Higgs inflation is special because of almost all properties are known or predictable! Below, I will argue that the SM in the Higgs phase does not suffer from a "hierarchy problem" and that similarly, the "cosmological constant problem" resolves itself if we understand the SM as a low energy effective theory emerging from a cutoff-medium at the Planck scale.

Adopting a bottom-up approach, I discuss these issues under the condition of a stable Higgs vacuum, by predicting the behavior of the SM when approaching the Planck era at high energies. SM Higgs inflation as exposed in this prelude may look pretty simple but in fact is rather subtle, because of the high sensitivity to the SM parameters and high sensitivity to higher order SM effects. In any case, my preconditions are: (i) a stable Higgs vacuum and a sufficiently large Higgs field at $M_{\mathrm{Pl}}$, (ii) physics beyond the SM should not spoil the main features of the SM. This means that SM extensions like SuperSymmetry (SUSY), or Grand Unified Theories (GUT) etc., pretending to solve the hierarchy problem and/or affecting the SM RG-flow substantially, are to be excluded! Here we have to assume that a kind of desert in the heavy particle spectrum is extending effectively up to the Planck scale. This is not so far beyond the "grand desert" usually assumed to exist in the context of GUTs. This does not exclude new physics that we know to exist, like dark matter, Majorana neutrinos or axions, for example.

Slow-roll inflation in general has been investigated in [25-30] in the 80's mostly as a top-down approach. An alternative "non-minimal gravity" Higgs-inflation approach has been advocated in [31-37]. Yet a different "eternal" Higgs inflation ansatz has been investigated within the context of superstring theory [38]. A time-dependent cosmological constant has been obtained also in a model which is based on a dilatation symmetry anomaly, where one assumes the Newton "constant" to be a time-dependent dynamical degree of freedom [39].

In this prelude, I have outlined what a correct interpretation of the "hierarchy problem" likely looks like, i.e. the predicted SM hierarchy pattern is not a problem, rather it is the solution for what we need to trigger inflation in the early universe. In the following I will consider the hierarchy issue in a broader context and discuss in some detail the intricacies of the cosmological constant problem and Higgs boson inflation. I will try to convince the reader that the Higgs boson inevitably delivers dark energy and the consequent inflation is well supported by a self-consistent perturbative SM calculation $[1,2]$. The approach is highly predictive and limited mainly by the uncertainties of the knowledge of the SM parameters and the accuracy of the perturbative calcula- 

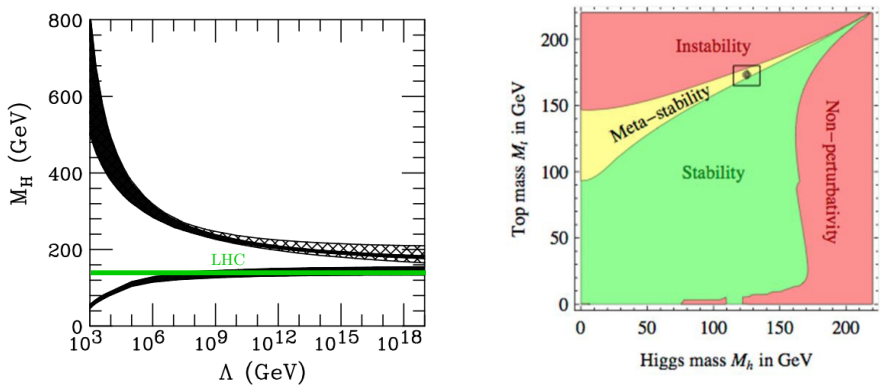

Figure 2: Left: the SM Higgs system remains perturbative up to $\Lambda_{\mathrm{Pl}}$ if $M_{H}$ is light enough (upper bound $=$ avoiding Landau pole) and the Higgs potential remains stable $(\lambda>0)$ if $M_{H}$ is not too light [parameters used: $M_{t}=$ 175[150 - 200] $\mathrm{GeV} ; \alpha_{s}=0.118$ ]. [Reprinted with permission from [50] http://dx.doi.org/10.1103/PhysRevD.55.7255. Copyright (1996) by the American Physical Society]. Right: A plot of the stability range in the $M_{H}-M_{t}$ plane. Courtesy of G. Degrassi et al. http://dx.doi.org/10.1007/JHEP08(2012)098, (License: CC-BY4.0). Reproduced from [58]

tions of the matching conditions between measured and $\overline{\mathrm{MS}}$ parameters and the $\overline{\mathrm{MS}}$ renormalization group coefficients.

\section{The Higgs boson discovery - the SM completion}

With the discovery of the Higgs boson by ATLAS [40] and CMS [41] in 2012 a last major but often questioned building block of the electroweak SM has been experimentally verified. The existence of an elementary scalar has been found to be required to render the electroweak massive gauge theory renormalizable in 1964 by Englert and Brout [42] and by Higgs [43]. The key mechanism turned out to be a Spontaneous Symmetry Breaking (SSB) mechanism of the non-Abelian $S U(2)_{L}$ gauge sector responsible for the weak interactions. The corresponding Higgs mechanism generates masses for all massive particles while not affecting the renormalizable UV behavior of the massless unbroken theory. Now, remarkably, the SM Higgs boson mass has been found in very special mass range $125.18 \pm 0.16 \mathrm{GeV}$, which seems to match the possibility to extrapolate the SM up to the Planck scale. Knowing the Higgs mass $M_{H}$ and using the mass coupling relation valid in the Higgs phase, we also know the Higgs self-coupling $\lambda$ and hence the renormalized Higgs potential $V=\frac{m^{2}}{2} H^{2}+\frac{\lambda}{24} H^{4}$, which is the object in our focus. Perturbativeness and vacuum stability of the Higgs potential are the key issues in this context (for early considerations see [44-49]). Fig. 2 adopted from an analysis by Hambye and Riesselmann in 1996 [50] illustrates the possible impact to have a Higgs mass in a window extending up to the Planck scale. The possibility that the SM may be extended right up to the Planck scale has been analyzed also in [51-57]. Later estimates have been improved after more precise SM parameters like the QCD coupling $\alpha_{s}$ and top-quark mass $M_{t}$ became available, see e.g. [58-62]. 
Given the Higgs self-coupling, all relevant SM parameters are known. While the RG evolution equations in the symmetric phase of the SM have been known for a long time to two loops, recently also the three-loop coefficients in the $\overline{\mathrm{MS}}$ scheme have been calculated [63-65]. The $\overline{\mathrm{MS}}$ input parameters, which are most suitable to parametrize the high energy tail, have to be calculated via the matching conditions from the experimentally measured ones (see $[58,61,62,66-71]$ and references therein). The matching conditions are based on more or less complete two-loop calculations that require the knowledge of the two-loop renormalization counterterms. Besides the electromagnetic vertex correction $\delta e$, all others are mass renormalization counterterms $\delta M_{b}^{2}$ for bosons and $\delta M_{f}$ for fermions, all given in terms of comparatively simple two-point functions, which are completely known to two loops ${ }^{4}$.

So far so good. One important point is missing however: the physical on-shell parameters are determined from experimental data by unfolding the raw data from radiation and detector effects. They represent pseudo-observables depending on theory input that relies on approximations. One has to keep in mind that most LEP, Tevatron and LHC results are based on incomplete two-loop calculations at best. Exceptions are the extraction of $G_{\mu}$ [74] and of $\sin ^{2} \Theta_{\text {eff }}^{\text {lept }}$ for which complete two-loop calculations exist [75]. Complete two-loop calculations have not been available at LEP times. For Bhabha scattering, which plays a key role for luminosity monitoring, the two-loop QED corrections became available only lately (see e.g. [76] and references therein). Full two-loop electroweak corrections to $2 \rightarrow 2$ processes either are still missing or have not been available when parameters were extracted from the data. There are persisting discrepancies at the $2 \sigma$ level in the determination of $\sin ^{2} \Theta_{\mathrm{eff}}^{\text {lept }}$ between SLD and LEP as well as for $\sin ^{2} \Theta_{\text {eff }}^{\mathrm{b} \bar{b}}$ and so there remain questions about the size of the estimated uncertainties of some of the input parameters.

Precision physics at LEP has been a great achievement also thanks to continuous progress in QCD and electroweak higher order calculations. Much progress has been achieved since. The SM is established with unprecedented accuracy. Activities now are focusing on LHC physics for obvious reasons. But more effort is needed to keep alive gained expertise on electroweak precision physics. Projects for future $e^{+} e^{-}$colliders like the International Linear Collider (ILC), which started with the unrealized TESLA project [77], and the Future Circular Collider (FCC) project at CERN, are boosting efforts to reach much higher precision in $Z$-peak, $W$-pair, top-quark and Higgs-boson physics [78]. The possibility that progress in this direction can establish the Higgs vacuum to be stable and the Higgs boson to be the inflaton in fact may be the strongest motivation to go on in realizing a next $e^{+} e^{-}$machine probing physics at the electroweak scale at much higher precision.

The most serious conceptual problem concerns the measurement of the top-quark mass and the related determination of the top Yukawa coupling. On the theory side, one is operating with the perturbative concept of an on-shell top-quark mass, which is

\footnotetext{
${ }^{4} \mathrm{~A}$ complete two-loop calculation has been performed independently by Veretin and Kalmykov in the context of [68]. Two-loop integrals exhibiting Higgs propagators have been expanded in $M_{V}^{2} / M_{H}^{2}$, assuming the Higgs to be heavier than the $W$ and $Z$ bosons. After the Higgs mass has been found this expansion turned out to be obsolete, and the relevant integrals had to be evaluated numerically. This has later been performed partially in [69] and "exact" in [72] such that a complete two-loop evaluation is available. For independent calculations of the matching conditions also see [73] and references therein.
} 
not an observable. Usually, the on-shell mass is identified with the Monte Carlo mass $M_{t}^{\mathrm{MC}}$. The latter is the kinematic mass parameter used Monte Carlo event generators that are utilized to extract the top-quark mass from top-quark production processes. The non-perturbative color screening effects obscure the precise determination of $M_{t}$ so far (see e.g. [62,79]). In addition, most of the Monte Carlo programs used at present do not take into account the electroweak radiative corrections.

My quintessence: while the matching conditions are known to two loops, the input parameters have not yet been determined at the same level of accuracy, i.e. likely reported errors are underestimated. These issues have to be reminded before one can claim that metastability of the electroweak vacuum is a proven fact.

\subsection{Matching conditions: $\overline{\mathrm{MS}}$ parameters in terms of physical pa- rameters}

We want to solve the RG equations up to very high scales, where mass effects are supposed to be negligible, in regions where physical thresholds play no role. The mass independent $\overline{\mathrm{MS}}$ scheme is the most simple choice, and under these circumstances likely a rather physical one, because it reflects the true UV structure of the SM in terms of quasi-bare quantities. In order to solve the $\overline{\mathrm{MS}} \mathrm{RG}$-equations we need the input $\overline{\mathrm{MS}}$ values of the basic couplings $g^{\prime}, g, g_{3}, y_{t}$ and $\lambda$. While $g_{3}\left(M_{Z}^{2}\right)=\sqrt{4 \pi \alpha_{s}\left(M_{Z}^{2}\right)}$ is a standard $\overline{\mathrm{MS}}$ reference parameter, the key parameters $y_{t}$ and $\lambda$ are known actually only through the related masses $M_{t}$ and $M_{H}$ :

$$
y_{t}^{2}=2 \sqrt{2} G_{\mu} M_{t}^{2}\left(1+\delta_{t}(\alpha, \cdots)\right) ; \lambda=3 \sqrt{2} G_{\mu} M_{H}^{2}\left(1+\delta_{H}(\alpha, \cdots)\right),
$$

where $\delta_{i}$ represent the corresponding radiative corrections. The relations between the renormalized on-shell masses and their $\overline{\mathrm{MS}}$ versions are provided by the natural matching conditions $m_{b \text { ren }}^{2}=M_{b \text { ren }}^{2}+\left(\delta M_{b}^{2}-\delta m_{b}^{2}\right)$, for bosons and $m_{f \text { ren }}=M_{f \text { ren }}+$ $\left(\delta M_{f}-\delta m_{f}\right)$, for fermions ${ }^{5}$. Formally we obtain them by writing the relation between the renormalized and the bare masses (in the bare Lagrangian) in the two schemes:

$$
m_{b \text { bare }}^{2} \stackrel{\text { OS }}{=} M_{b \text { ren }}^{2}+\delta M_{b}^{2} \stackrel{\overline{\mathrm{MS}}}{=} m_{b \text { ren }}^{2}+\delta m_{b}^{2}
$$

for bosons and

$$
m_{f \text { bare }} \stackrel{\mathrm{OS}}{=} M_{f \text { ren }}+\delta M_{f} \stackrel{\overline{\mathrm{MS}}}{=} m_{f \text { ren }}+\delta m_{f},
$$

for fermions, i.e. the mass shift is given by the $\overline{\mathrm{MS}}$ finite part prescription applied to the (UV singular) OS mass counterterm:

$$
\left.m_{b}^{2}\right|_{\overline{\mathrm{MS}}}=\left.M_{b}^{2}\right|_{\mathrm{OS}}+\left.\delta M_{b}^{2}\right|_{\frac{2}{\varepsilon}-\gamma+\ln 4 \pi+\ln \mu_{0}^{2} \rightarrow \ln \mu^{2}},
$$

\footnotetext{
${ }^{5}$ We denote on-shell masses by capital, $\overline{\mathrm{MS}}$ masses by lower case letters as in [1]
} 
for bosons and

$$
\left.m_{f}\right|_{\overline{\mathrm{MS}}}=\left.M_{f}\right|_{\mathrm{OS}}+\left.\delta M_{f}\right|_{\frac{2}{\bar{\varepsilon}}-\gamma+\ln 4 \pi+\ln \mu_{0}^{2} \rightarrow \ln \mu^{2}},
$$

for fermions.

Here it is important to keep in mind: for the renormalized theory the $\overline{\mathrm{MS}}$ parametrization is not a parametrization in terms of observables but serves as a convenient intermediate (auxiliary) parametrization. The $\overline{\mathrm{MS}}$ scheme is a purely perturbative concept (no non-perturbative definition) and corresponding parameters are not measurable.

However, if we take the bare theory to be the physical one in the sense of a low energy effective theory exhibiting a physical cutoff, then the $\overline{\mathrm{MS}}$ parameters in the perturbative regime are representing the bare parameters. Crucial for the extrapolation to the Planck scale, if possible, it is to keep the relationships between bare, $\overline{\mathrm{MS}}$ and physical OS parameters gauge invariant and preserving the UV structure (see e.g. [66, 67]).

So in principle, on a conceptual level, we are confronted with a well-defined problem of calculating the massive physical particle self-energies exact to two loops, in addition to the e.m. vertex, in order to find the appropriate input for the three-loop $\overline{\mathrm{MS}}$ RG running.

In the symmetric phase of the SM, except the $m^{2}$-term in the Higgs potential, all masses vanish and the RG coefficient calculations and the solution of the RG equations are straight forward. This insofar that there are no special mass-coupling relations and the decoupling of heavy states (the four Higgs scalars) is given as requested by the Appelquist-Carazzone theorem (AC) [80], which in the broken phase only holds in the QCD and QED sectors.

In contrast, in the Higgs phase of the SM, there are some tricky points to be taken care of.

1) The tadpole issue: if we require the bare parameters, now represented by the $\overline{\mathrm{MS}}$ parameters, as physical we have to respect Ward-Takahashi and Slavnov-Taylor identities. This requires to take into account tadpole contributions according to Fig. 3, which mostly are omitted in calculations (see e.g. [71,73, 81]), because a theorem [82, 83] states that tadpoles cancel in physical quantities provided they are expressed in terms of physical quantities within the renormalized theory (see [69] for a recent discussion).

2) The lack of decoupling issue: while in QED and QCD, heavy particles decouple, within the SM heavy states do not decouple when the mass-coupling relations come into play. Masses and couplings are one-to-one interrelated, because all masses are generated by the Higgs mechanism. For the given VEV $v$ a mass can only get large iff the corresponding coupling gets large. The couplings are active at scales below the related mass thresholds. Within the $\overline{\mathrm{MS}}$ scheme, which respects gauge invariance but has deficiencies like the lack of decoupling of heavy states, decoupling has to be imposed by hand, and one is working with effective field theory including only the active flavors at the given scale, in place of full theory. Most of the matching analyses are inspired by techniques that are well established in QCD. Typically, one is matching the $N_{f}$ with the $N_{f}+1$ flavor effective QCD at the $N_{f}+1$ flavor threshold. This is well justified in the time-like regime where corresponding thresholds are manifest. While physical observables are naturally sharing decoupling properties, within the full SM 


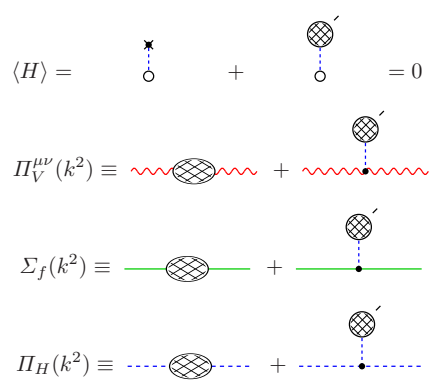

Figure 3: Tadpoles show up in the broken phase as diagrams contributing to the Higgs field VEV. They contribute to self-energies as depicted. Tadpoles are gauge dependent and UV singular and have to be taken into account as shown in order to preserve the gauge symmetry [66].

the lack of automatic decoupling is a serious shortcoming of the $\overline{\mathrm{MS}}$ parametrization when applied below the highest SM threshold at $2 M_{t}$.

The most prominent non-decoupling effect is due to the large top Yukawa coupling, which we know to be interrelated with the heavy top-quark mass. It yields the leading correction of the EW $\rho$-parameter, defined by the neutral to the charged current ratio of the corresponding low energy effective Fermi couplings, which is given by

$$
\rho=G_{\mathrm{NC}} / G_{\mathrm{CC}}(0)=1+\frac{N_{c} G_{\mu}}{8 \pi^{2} \sqrt{2}}\left(m_{t}^{2}+m_{b}^{2}-\frac{2 m_{t}^{2} m_{b}^{2}}{m_{t}^{2}-m_{b}^{2}} \ln \frac{m_{t}^{2}}{m_{b}^{2}}\right) \approx 1+\frac{N_{c} y_{t}^{2}}{32 \pi^{2}},
$$

where $N_{c}=3$ is the number of colors. It is quadratic in $y_{t}$ and measures weak-isospin breaking by the Yukawa couplings of the heavy fermions at zero momentum. So, the top Yukawa coupling is at work down to zero momentum and not being active starting above the top mass threshold only. This type of effect measured for the first time at LEP in 1995 far below the top-pair threshold, allowed to derive a bound on $M_{t}$ before the top-quark discovery at the Tevatron in 1996.

This shows that "decoupling by hand" cannot be applied for weak contributions, i.e., we cannot parametrize and match together effective theories by switching off fields of mass $M>\mu$ at a given scale $\mu$.

Only a direct measurement of $y_{t}$ and $\lambda$ at a facility like FCC-ee or ILC above the top-quark mass threshold can provide us the precise input parameters we need.

3) The Fermi constant issue: the Higgs-field VEV $v$ determines the Fermi constant via

$$
G_{F}=\frac{1}{\sqrt{2} v^{2}} \text { or } \sqrt{2} G_{\mu}=v^{-2}=\frac{e^{2}}{4} \frac{M_{Z}^{2}}{M_{W}^{2}} \frac{1}{M_{Z}^{2}-M_{W}^{2}} .
$$

For the on-shell counterterm we then have the relation $\left(c_{W}^{2}=M_{W}^{2} / M_{Z}^{2}, s_{W}^{2}=1-c_{W}^{2}\right)$

$$
\frac{\delta G_{F}}{G_{F}}=2 \frac{\delta v^{-1}}{v^{-1}} \text { and } \frac{\delta v^{-1}}{v^{-1}}=\frac{\delta e}{e}-\frac{1}{2 s_{W}^{2}}\left(s_{W}^{2} \frac{\delta M_{W}^{2}}{M_{W}^{2}}+c_{W}^{2} \frac{\delta M_{Z}^{2}}{M_{Z}^{2}}\right) .
$$


Potentially, the Higgs-field VEV $v$ could be particularly affected by non-decoupling effects. However, here we may take advantage of the fact that tadpole contributions drop out in relations between physical (on-shell) parameters and physical transition amplitudes. To be compared are

- low energy $-G_{\mu}=G_{\mu}\left(Q^{2} \approx 0\right)$ determined by the muon lifetime,

- $W$ mass scale $-\hat{G}_{\mu}=G_{\mu}\left(Q^{2} \approx M_{W}^{2}\right)=\frac{12 \pi \Gamma_{W \ell}}{\sqrt{2} M_{W}^{3}}$ given by leptonic $W$ decay rate. Indeed $\hat{G}_{\mu} \approx G_{\mu}$ with good accuracy as expected ${ }^{6}$.

Finally we need the $\overline{\mathrm{MS}}$ version of $G_{F}$. Again, evaluating $G_{F \text { bare }}=G_{\mu}+\delta G_{F}$ in both schemes we have

$$
G_{F}^{\overline{\mathrm{MS}}}=G_{\mu}+\left(\left.\delta G_{F}\right|_{\mathrm{OS}}\right)_{\frac{2}{\varepsilon}-\gamma+\ln 4 \pi+\ln \mu_{0}^{2} \rightarrow \ln \mu^{2}} .
$$

We calculate it equivalently by using (13) in the respective schemes. Then the $\overline{\mathrm{MS}}$ top-quark Yukawa coupling is given by

$$
y_{t}^{\overline{\mathrm{MS}}}\left(M_{t}^{2}\right)=\sqrt{2} \frac{m_{t}\left(M_{t}^{2}\right)}{v^{\mathrm{MS}}\left(M_{t}^{2}\right)} ; v^{\overline{\mathrm{MS}}}\left(\mu^{2}\right)=\left(\sqrt{2} G_{F}^{\overline{\mathrm{MS}}}\right)^{-1 / 2}\left(\mu^{2}\right) .
$$

A good matching scale is $M_{Z}$ using $\alpha\left(M_{Z}\right), \alpha_{s}\left(M_{Z}\right)$ and $\hat{G}_{\mu}$ as input. The non-perturbative contribution $\Delta \alpha_{\text {had }}^{(5)}\left(M_{Z}^{2}\right)$ to the shift in $\alpha\left(M_{Z}\right)$ is taken from [84]. I have outlined the points that can lead to slightly different input values for the $\overline{\mathrm{MS}}$ parameters as listed in Table 1. Our evaluation is documented in more detail in Refs. [1,2,69]. One difference concerns the use of the Fermi constant, which in a number of analyses is taken to be the low energy $G_{\mu}$, assumed not to be running below the top-quark mass threshold, and often a difference between its OS and $\overline{\mathrm{MS}}$ version is not made (see e.g. $[58,70])$. A second cause for a difference is related to a different account of the tadpole contributions (see e.g. [71, 73]). Given the $\overline{\mathrm{MS}}$ input, the RG-equations are then solved in the $\overline{\mathrm{MS}}$ scheme to three loops by also including four- and five-loop results in the QCD sector. Concerning the RG-running, there is full agreement between the different studies available in the literature for a given set of input parameters. The only parameter which does not agree within quoted uncertainties is the top-quark Yukawa coupling $y_{t}$ (9), which apparently is the parameter which decides about the stability of the vacuum.

Another important point, which is only partially taken care of in estimating the uncertainties of the physical input parameters, is the following:

4) The scheme dependence issue: perturbative predictions are renormalization scheme dependent due to truncation errors of the perturbative expansion. A typical example is provided by the electromagnetic fine structure constant. At very low energy the value in the Thomson limit $\alpha \simeq 1 / 137$ is adopted as an input parameter, while at the $Z$ boson mass scale $\alpha^{\prime}=\alpha\left(M_{Z}\right)=\frac{\alpha}{1-\Delta \alpha\left(M_{Z}\right)} \simeq 1 / 127$ is a more appropriate input parameter, because it represents an universal type large correction requiring RG resummation, and which enters radiative correction calculations at many places. To one-loop order

\footnotetext{
${ }^{6} \mathrm{~A}$ LO estimate with $M_{W}=80.385 \pm 0.015 \mathrm{GeV}, \Gamma_{W}=2.085 \pm 0.042 \mathrm{GeV}$ and $B\left(W \rightarrow \ell v_{\ell}\right)=10.80 \pm$ $0.09 \%$ yields $\hat{G}_{\mu}=1.15564(55) \times 10^{-5} \mathrm{GeV}^{-2}$ to be compared with $G_{\mu}=1.16637(1) \times 10^{-5} \mathrm{GeV}^{-2}$, i.e. the on-shell Fermi constant at scale $M_{Z}$ appears reduced by $0.92 \%$ relative to $G_{\mu}$.
} 
$\alpha^{\prime}=\alpha(1+a \alpha)$. If we calculate a matrix-element including one-loop corrections

$$
M^{(1)}=\alpha^{n} C(1+b \alpha),
$$

in terms of $\alpha^{\prime}$ we obtain

$$
M^{\prime(1)}=\alpha^{\prime n} C\left(1+b^{\prime} \alpha^{\prime}\right),
$$

and hence

$$
M^{(1)}=M^{(1)}+\delta M,
$$

where, inserting $b^{\prime}=b-n a$,

$$
\delta M=\alpha^{n} C\left(\left(\frac{1}{2} n(n-1) a^{2}+(n+1) a b^{\prime}\right) \alpha^{2}+\cdots+a^{n+1} b^{\prime} \alpha^{n+2}\right) .
$$

Thus the result differs by $\delta M$. If we do not actually calculate the higher orders

$$
\delta M=M^{(1)}-M^{(1)}
$$

must be considered as an uncertainty due to unknown higher order effects. Actually, results can differ non-negligibly when different parameter sets are used as independent input parameters, like $\alpha, M_{W}, M_{Z}, \cdots, G_{\mu}, M_{W}, M_{Z}, \cdots$ or the preferred LEP parametrization in terms of the most precisely known parameters $\alpha, G_{\mu}, M_{Z}, \cdots$, the scheme which is usually applied (see e.g. [85]).

I am mentioning the scheme dependence uncertainties here because, to my knowledge, they are not fully taken into account in standard extractions of on-shell parameters from the data. One more reason why strong statements concerning a proof of metastability and "just failing vacuum stability", it seems to me can hardly be justified in view of the strong sensitivity of vacuum stability on precise input parameters.

I remind that the most important difference between my "cutoff extended" SM and the most often discussed metastability path to high energies (discussed within the framework of the renormalized SM) lies in the fact the I have to take care that my $\overline{\mathrm{MS}}$ parametrization is equivalent to the bare parametrization. This is what makes the inclusion of tadpoles mandatory. We have explicitly checked in [68] that only by including tadpoles the $\overline{\mathrm{MS}} \mathrm{RG}$ equations in the broken phase agree with the ones in the symmetric phase ${ }^{7}$.

\subsection{The SM running parameters}

In Fig. 4 we plot the evolution of the SM couplings as a function of the log of the energy scale. As we learn from Fig. 4 the amazing thing is that the perturbation expansion turns out to work up to the Planck scale! In our analysis, for the input parameters specified below, we have no Landau pole or other singularities and the Higgs potential remains stable. This likely opens a new gate to precision cosmology of the

\footnotetext{
${ }^{7}$ Obviously, in electroweak theory, what is called $\overline{\mathrm{MS}}$ scheme may refer to two different versions, depending on whether tadpoles are dropped or not [81]. This only concerns calculations in the broken phase where mass effects play a role, as for the matching conditions (see [69]). Note that RG coefficients are calculated in the massless symmetric phase where they are unambiguous, since tadpoles are absent.
} 


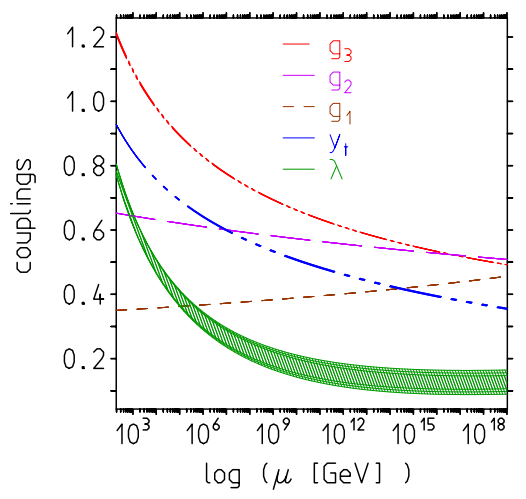

Figure 4: The SM dimensionless couplings in the $\overline{\mathrm{MS}}$ scheme as a function of the log of the renormalization scale for $M_{H}=124-126 \mathrm{GeV}$ (shaded band). Input parameters as in Table 1

early universe $[1,58,61]$. The remarkable interrelations between SM couplings may be summarized as follows: the $U(1)_{Y}$ coupling $g_{1}$ is screening (IR free), the $S U(2)_{L}$ coupling $g_{2}$ and the $S U(3)_{c}$ coupling $g_{3}$ are anti-screening (UV free=Asymptotic Freedom (AF)) as expected [86]. In contrast the top-quark Yukawa coupling $y_{t}$ and the Higgs self-coupling $\lambda$, which are screening if standalone (IR free, like QED), within the SM change their behavior from IR free to UV free, such that perturbation theory works the better the higher the energy in these couplings as well. What happens is that QCD effects dominate the behavior of the top-quark Yukawa coupling RG provided $g_{3}>\frac{3}{4} y_{t}$ in the gaugeless $\left(g_{1}, g_{2}=0\right)$ approximation, which is satisfied. Similarly, the top-quark Yukawa effect dominates the Higgs coupling RG provided $\lambda<\frac{3}{2}(\sqrt{5}-1) y_{t}^{2}$, which also holds in the gaugeless $\left(g_{1}, g_{2}=0\right)$ limit. These conditions are satisfied in the SM with the given parameters and extend to higher orders as far as these are known. We note that the Abelian hypercharge coupling $g_{1}$, although increasing with energy, stays small up to $\Lambda_{\mathrm{Pl}}$ such that it does not affect perturbativeness. Note that in spite of its increasing behavior $g_{1}<g_{3}<g_{2}$ at Planck scale. Interestingly there we have an inverted $g_{3}<g_{2}$ hierarchy of the non-Abelian gauge couplings. In the focus is the Higgs selfcoupling, because it may not stay positive $\lambda>0$ up to $\Lambda_{\mathrm{Pl}}$. In fact a $3 \sigma$ significance for meta-stability is claimed e.g. in $[58,61]$ (see right panel of Fig. 2). Calculating previously missing two-loop contributions to the matching conditions the significance for missing stability could be reduced to a $1 \sigma$ gap in [71]. The existence of a zero in $\lambda(\mu)$ crucially depends on the precise size of the top-quark Yukawa coupling $y_{t}$, which actually seems to decide about the stability of our world. Note that $\lambda=0$ would be an essential singularity! Uncertainties here have to be reduced by more precise input parameters and better established EW matching conditions. For our input parameters, the Higgs coupling decreases up to the zero of the beta-function $\beta_{\lambda}$ at $\mu_{\lambda} \sim 3.5 \times 10^{17} \mathrm{GeV}$, where $\lambda$ is small but still positive and above $\mu_{\lambda}$ increases with energy up to $\mu=M_{\mathrm{Pl}}$.

I think our discussion shows that ATLAS and CMS results may have revolutionized particle physics in an unexpected way, namely showing that the SM has higher 
self-consistency (conspiracy) than expected and previous arguments for the existence of new physics may turn out not to be compelling. Also, the absence so far of any established new physics signal at the LHC may indicate that commonly accepted expectations may not be satisfied. On the one hand, it seems to look completely implausible to assume the SM to be essentially valid up to Planck energies, on the other hand, the flood of speculations about physics beyond the SM have been of no avail. Within the context of GUTs, a large gap in the particle spectrum, the "grand desert" up to the GUT scale at about $10^{16} \mathrm{GeV}$, still is a widely accepted hypothesis. So why the "grand desert" could not extend a little further namely to $10^{19} \mathrm{GeV}$ ? The central issue for the future is the very delicate "acting together" between SM couplings, which makes the precision determination of SM parameters more important than ever. This mainly challenges accelerator physics, the LHC experiments, and the future ILC and FCC-ee projects (top-quark and Higgs-boson factories), which could improve the precision values for $\lambda, y_{t}$ and $\alpha_{s}$. Still important are lower energy hadron facilities, which should provide more precise hadronic cross sections in order to reduce hadronic uncertainties in $\alpha\left(M_{Z}\right)$ and $\alpha_{2}\left(M_{Z}\right)$. This could open a new gate to precision cosmology of the early universe, in case the Higgs boson inflation scenario outlined in Sect. 1 could be hardened.

\section{Thoughts on guiding principles and paradigms in par- ticle physics}

In last decades "solving the hierarchy problem" has been a strong motivation to find possible extensions of the SM. Guiding principles often have played an important role in progress in science although they afterward turned out to miss the point they suggested natural laws should follow. Most prominent are symmetry principles. Related group theory is beautiful mathematics but is not always mapping the real or supposed physical problem it was proposed to describe. Kepler already dreamed of the Platonic bodies (regular polyhedra) to rule celestial mechanics of planets. After his attempt to prove this by analyzing celestial data, finally, Kepler's laws resulted from his investigation. Kepler's model is completely false, the interplanetary distances it predicts are not sufficiently accurate, and Kepler was scientist enough to accept this eventually. But it is an excellent example of how truth and beauty do not always fit together. The widespread string theory paradigm assumes that a simple highly symmetric stringy structure at and beyond the Planck scale ${ }^{8}$ could explain what we observe down on earth, actually a rather complex real world. That something simple looks complicated when seen from far away is certainly not a very natural expectation. A solid macroscopic may look to be perfectly rotational symmetric, zooming in on its microscopic structure can uncover a lattice of atoms exhibiting all kinds of lattice defects and domain pat-

\footnotetext{
${ }^{8}$ String theory is motivated by the requirement to 'quantize gravity'. Note that in string theory the Planck energy level represents the ground state on top of which an infinite tower of harmonics at $E_{n}=n M_{\mathrm{Pl}}(n=$ $2, \cdots, \infty)$ resides. The algebra of the spectral raising and lowering operators can be closed to a Kac-Moody algebra (infinite dimensional analogs of semi-simple Lie algebra) only in a particular space-time dimension $D$. The unique supergravity string theory requires $D=11$, where 7 of them are assumed to be compactified (see e.g. [87]). For me it is hard to believe that this is what shapes our real world.
} 
terns. The "the closer you look the more there is to see" pattern of thought looks to me less artificial than expecting some harmonic oscillator "heaven". In any case concepts like the ones we are discussing here: Naturalness, Hierarchy and Fine-Tuning make no sense without specifying the context in which they are addressed.

While "solving the hierarchy problem" seems to fail as a route to new physics, in contrast, the concept of the minimal renormalizable extension of Fermi's weak interaction theory turned out to be impressively successful in constructing piece by piece the electroweak SM. It has lead to the introduction of the massive intermediate spin 1 bosons $W^{ \pm}$in charged current processes, the prediction of neural currents and the need for a $Z$ boson. The resulting $U(1)_{Y} \otimes S U(2)_{L}$ gauge theory, renormalizable in the massless case, requires a scalar spin 0 boson, the Higgs boson, as a trick to generate masses of the weak gauge bosons and the fermions, without spoiling renormalizability. Spontaneous symmetry breaking, a mechanism known from condensed matter physics, turned out to be the key mechanism for a renormalizable massive gauge theory. Another example concerning minimality versus non-minimality we have when considering GUTs, where fermions are necessarily populating higher representations while the fundamental ones are not occupied. Therefore the typical leptoquarks necessarily showing up in GUTs are unnatural as an emergent phenomenon ${ }^{9}$.

A convincing solution of the SM's hierarchy problem is known to be provided by a supersymmetric extension of the SM. SUSY cannot be an exact symmetry because it would predict a degenerate mass spectrum while phenomenologically the states of the SUSY mirror world all must be heavier than the SM particles. This leads to a very complicated world as a broken SUSY scenario not only is doubling the spectrum at once but also leaving too much freedom, with about 100 unknown symmetry breaking parameters. This makes such extensions not really predictive without additional assumptions. At the end phenomenological constraints require a SUSY version that would not be solving the hierarchy problem really, rather it would only be shifting the amount of fine-tuning required.

In addition, the hierarchy fine-tuning problem if being solved by a supersymmetrization of the SM creates new problems as we know. First, a second Higgs doublet field needs to be introduced, which as such is an interesting option. However, in order not to be in conflict with the absence of tree-level Flavor Changing Neutral Currents (FCNC) ${ }^{10}$ one has to impose $R$-parity, which is not less a fine tuning, although FCNCs can be forbidden by a simple discrete symmetry. $R$-parity is not required by renormalizability, it is not naturally emergent in a low energy effective theory and thus looks to be ad hoc ${ }^{11}$. A generic SUSY extension as such would be in contradiction with obser-

\footnotetext{
${ }^{9}$ The unification paradigm celebrated its triumphal success in Maxwell's electromagnetism, which unified electrical and magnetic laws and predicted electromagnetic waves. In contrast, as we know the electroweak theory is not a true unification, it rather regulates the mixing of electromagnetic and weak interaction phenomena. At the heart is $\gamma-Z$ mixing and $Z$ resonance (a kind of "heavy-light") physics, which manifests itself most convincingly in electron-positron annihilation into $Z$ bosons. All further unification attempts so far are missing confirmation.

${ }^{10}$ FCNCs are automatically absent in the SM by the GIM mechanism [88], as it is also highly established by experiment.

${ }^{11}$ Similarly, a global lepton flavor symmetry $U(1)_{e} \otimes U(1)_{\mu} \otimes U(1)_{\tau}$, which would imply exact lepton flavor conservation is not emergent because renormalizability does not dependent on it. That it is a surprisingly accurate approximate symmetry is due to the smallness of the neutrino masses, which likely results as a
} 
vation right away. This also illustrates that naturalness is a doubtful concept: $R$-parity is a symmetry which forbids FCNCs but what is natural about it?

In my opinion, the dogma surrounding the so-called hierarchy or fine-tuning problem turned out to be a complete failure. Similarly, the GUT paradigm has not been leading to any experimentally confirmed predictions that would support this concept. I think the minimal renormalizable QFT paradigm is back. Thereby "minimal" is crucial, many higher renormalizable structures like a GUT extension of the SM are not natural in that sense. Interestingly, a missing third fermion family or an additional fourth family would spoil important properties of the SM, such as the RG flow, and the Higgs boson then could not be a candidate for the inflaton. Another very important special feature of the SM is its tree level accidental custodial symmetry. The latter is violated by many of the proposed extensions of the SM, which then create a different fine tuning problem [89], in all cases that violate the tree level SM relation $\cos ^{2} \Theta_{W} M_{Z}^{2} / M_{W}^{2}=1$. This relation is strongly supported by experimental data, which precisely confirm SM predictions of the radiative corrections.

One also has to keep in mind that precision tests of the SM already revealed a test in-depth of its quantum structure. The largest corrections come from the running fine structure constant $\alpha(s)$, the running of the strong coupling $\alpha_{s}(s)$ and the large top Yukawa $y_{t}^{2}(s)$ effects. As contribution to the $\rho=G_{\mathrm{NC}} / G_{\mu}(0)$ parameter, for example, subleading corrections amount to a $10 \sigma$ deviation from the SM prediction when taking into account the largest corrections only. Thus the SM is on very solid grounds better than everything else we ever had.

On the other hand, the view that the SM is a low energy effective theory of some cutoff system at the Planck energy scale $M_{\mathrm{Pl}}$ appears to be consolidated. This also puts QFT on a firm mathematical basis. A crucial point is that $M_{\mathrm{Pl}}$, providing the scale for the low energy expansion in powers $E / M_{\mathrm{Pl}}$, is exceedingly large, very far from what we can see! A dimension 6 operator at LHC energies is suppressed by $\left(E_{\mathrm{LHC}} / \Lambda_{\mathrm{Pl}}\right)^{2} \approx$ $10^{-30}$. This seems to motivate a change in paradigm from the view that the world looks simpler the higher the energy to a more natural scenario which understands the "cutoff SM" as the "true world" seen from far away, with symmetries emerging from not resolving the details of the short distance structure. In the low energy expansion, one is "throwing away" an infinite tower of shorter distance information carried by the suppressed so-called irrelevant operators.

The hierarchy problem requires to take the relationship between the bare UV and the renormalized IR regime as testable physics. Here Wilson's RG comes into play. Kenneth Wilson 1971 [90] has been able to solve the problems surrounding the critical indices of phase transitions in condensed matter systems, which have been persisting for about 75 years. His work has shed new light on the role cutoffs may play in physical laws. Wilson's renormalization semi-group, based on integrating out irrelevant details of the short distance structure opened the quantitative approach of constructing low energy effective quantum field theories that derive from systems whose short distance structure has an intrinsic cutoff, like an atomic lattice or an atomic gas or fluid (see e.g. [91]). The key low energy emergent structure notably turned out to reveal renormalizable Euclidean quantum field theory. The latter exhibits analyticity

consequence of a see-saw mechanism at work. 
in a way which makes it equivalent to a Minkowski quantum field theory. The latter hence is incorporating quantum mechanics as an emergent structure. As I will argue in the following, cutoffs in particle physics are unavoidable in understanding the relationship between a bare and a renormalized theory (see e.g. [92]). In such context, renormalizability is an emergent property like all structures required in order renormalizability to be manifest. In our context cutoffs are indispensable for understanding early cosmology in a bottom-up way [1]. This opens the possibility of an alternative understanding of inflation, reheating, baryogenesis and all that [25-30]. As in condensed matter physics the connection between macroscopic long distance physics (at laboratory scales) and the microscopic underlying cutoff system (high energy events as they were natural in the early universe) turn out to have a physical meaning.

I remind that the SM's naturalness problems and fine-tuning problems have been made conscious by G. 't Hooft [3] long time ago as a possible problem in the relationship between macroscopic phenomena that follow from microscopic laws (a condensed matter system inspired scenario). Soon later the "hierarchy problem" had been dogmatized as a kind of fundamental principle. In fact, the hierarchy problem of the SM had been the key motivation for a number of types of extensions of the SM. It is therefore important to reconsider the "problem" in more detail.

One of my key points concerns the different meaning a possible hierarchy problem has in the symmetric and in the broken phase of the SM. In order to understand the point, we have to remember why we need the Higgs particle in the SM. The Higgs boson is necessary to get a renormalizable low energy effective electroweak theory [10]. Interestingly, one scalar particle is sufficient to solve the renormalizability problems arising from each of many different massive fields in the SM, of which each causes the problem independently of the others. The point is that this one particle has to exhibit as many new forces as there are individual massive states [11]. All required new interactions are in accordance with the SM symmetry structure in the symmetric phase as we know. The taming of the high energy behavior, of course, requires the Higgs boson to have a mass in the ballpark of the other given heavier SM states, if it would be much heavier it would not serve its purpose in the low energy regime. It would lead to the so-called "delayed unitarity" phenomenon [93]. Note that the Higgs boson has to cure the unphysical mass effects for the given gauge boson masses $M_{W}, M_{Z}$ and fermion masses $M_{f}$ via adequate Higgs exchange forces, where the coupling strength is proportional to the mass of the massive field coupled. A very heavy Higgs boson eventually would decouple and thus miss to restore renormalizability of the massive vector-boson gauge theory. Interestingly, in the symmetric phase the SM gauge-boson plus chiral fermions sector is renormalizable without the Higgs-boson and Yukawa sectors and scalars are not required at all to cure the high energy behavior because it is renormalizable by its own structure. Therefore, in the symmetric phase, the mass degenerate Higgs fields in the complex Higgs doublet can be as heavy as we like. Since unprotected by any symmetry, naturally we would expect the Higgs particles indeed to be very heavy. In fact the "origin" of the Higgs mass is very different in the broken phase, where all the masses, including the Higgs mass itself, are generated by the Higgs mechanism [42,43]. This we learn from the relation $m_{H}^{2}=\frac{1}{3} \lambda v^{2}$, holding in the broken phase. In the symmetric phase, the effective Higgs mass is dynamically generated by the Planck medium, as we will argue below. Therefore, the usual claim that the 
SM requires to be extended in such a way that quadratic divergences are absent has no foundation. Purely formal arguments based on perturbative counterterm adjustments do not lead any further.

The hierarchy problem in particular addresses the presence of quadratic UV divergences related to the SM Higgs mass term. Infinities in physical theories are the result of idealizations and show up as singularities in a formalism or in models. UV singularities in general plague the precise definition as well as concrete calculations in quantum field theories $(\mathrm{QFT})^{12}$. A closer look usually reveals infinities to parametrize our ignorance or mark the limitations of our understanding or knowledge. One particular consequence of UV divergences in local QFT is that the vacuum energy is ill-defined as it is associated with quartically divergent quantum fluctuations.

This is another indication that tells us that local continuum QFT has its limitation and that the need for regularization is actually the need to look at the true system behind it. In fact the cutoff system is more physical and does not share the problems with infinities resulting from the idealization realized in the large cutoff limit or lattice continuum limit. In any case, the framework of a renormalizable QFT, which has been extremely successful in particle physics up to highest accessible energies is not able to give answers to the questions related to vacuum energy and hence to all questions related to dark energy, accelerated expansion, and inflation of the universe.

Since the SM exhibits non-AF couplings like the $U(1)_{Y}$ coupling $g_{1}$ or the Higgs self-coupling $\lambda$ at scales beyond the zero of the $\beta_{\lambda}$ function, also lattice calculations [9698] strongly suggest that in fact, the theory requires a finite cutoff, because the continuum limit at infinite cutoff would be trivial.

It is thus natural to consider the SM to be what we observe as the Low Energy Effective SM (LEESM), the renormalizable tail of the real cutoff system sitting at the Planck scale. As a consequence all properties required by renormalizability, gauge symmetries, chiral symmetry, anomaly cancellation, and the related fermion family structure, as well as the existence of an elementary scalar, the Higgs boson, naturally emerge as a consequence of the low energy expansion ${ }^{13}$. We remind that the emergence of SM structures in a low energy expansion is a well investigated subject (see e.g. [99] and references therein). It is often advocated as a tree-unitary requirement but is easily reinterpretable as a low energy expansion where non-renormalizable effects are suppressed by inverse powers in the cutoff. These mechanisms are calculable within perturbation theory [100-106]. As SM perturbation theory works at the $Z$ mass scale and gets better with increasing energy these perturbative derivations of gauge symmetry and Higgs structure attain the status of proofs. The infinite tower of higher order operators is suppressed to be invisible. Only a few operators are non-irrelevant

\footnotetext{
${ }^{12}$ Taming the infinities we encounter in the theory of elementary particles, i.e. of quantum field theories, by completing them with a cutoff, often called the UV-completion of a QFT is as old as QFT itself. Actually, it took 20 years from Dirac 1928 (Dirac hole theory of relativistic electron-photon interaction [pre-QED]) to Feynman, Schwinger and Tomonaga in 1948 who found how to deal with the large cutoff limit and making QED a predictive theory. For non-Abelian gauge theories proposed by Yang and Mills in 1954 [94] it took another 17 years until a renormalizable formulation was found by 't Hooft in 1971 [95] (actually by circumventing a cutoff regularization).

${ }^{13}$ It is interesting to note that statistical mechanical systems with short-range exchange and long-range multipole interactions exhibit vector bosons and graviton modes that follow from a multipole expansion of a static potential [100]. In this sense the emergence of gauge-bosons looks pretty natural.
} 
and effectively observable, and this is what makes the world look much simpler than a possibly chaotic Planck medium. In reality, infinities related to the relevant operators are replaced by eventually very large but finite numbers, and I will show that sometimes such huge effects are needed in order to understand the real world. I will argue that cutoff enhanced effects are responsible for triggering the Higgs mechanism not very far below the Planck scale and the inflation of the early universe, as outlined already in Sect. 1.

The history of our universe we can trace back 13.8 billion years close to the Big Bang, when the expansion of the universe was ignited in a "fireball", an extremely hot and dense state when all structures, and in the end, all atoms, nuclei, and nucleons were disintegrated into a world of elementary particles only. So the SM provides the key information for what has happened in the early universe, and high energy accelerator experiments are testing processes that only took place in nature in the early history of the universe. If the Higgs boson is the source of dark energy that triggered inflation, its discovery could mark a milestone in our understanding of the dynamics of the very early universe. The origin of cold dark matter remains a mystery, which can have many different explanations.

I think that questions concerning the early universe can be addressed only within a LEESM "extension" of the SM, e.g. given by the SM supplied with a cutoff structure in a minimal way. As we know, in a renormalizable QFT all renormalized quantities as a function of the renormalized parameters and fields in the limit of a large cutoff are finite and devoid of any cutoff relicts! Here we should remember the BogoliubovParasiuk renormalization theorem that states that order by order in perturbation theory the renormalized Green's functions and matrix elements of the scattering matrix (Smatrix) are free of ultraviolet divergences. The theorem specifies a concrete procedure (the Bogoliubov-Parasiuk R-operation) for the subtraction of divergences, establishes the correctness of this procedure, and guarantees the uniqueness of the obtained results, modulo reparametrizations, which are controlled by the renormalization group. In other words, in the low energy world cutoff effects are not accessible to experiments. Consequently, the hierarchy problem cannot be addressed within the renormalizable, renormalized SM, which encodes all observables. In this framework, all independent parameters are free and have to be supplied by experiments. In this sense, within the renormalized QFT the hierarchy problem is a pseudo-problem.

To my knowledge, the only non-perturbative definition of a renormalizable local quantum field theory is the possibility to put in on a lattice by discretization of spacetime. This again may be taken as an indication that the need for a cutoff actually is an indication that the cutoff exists in the real world. In this sense, lattice-QFT is closer to the true system than its continuum tail. Of course, there are many ways to introduce a cutoff and actually, we cannot know what the cutoff system looks like truly. This is not a real problem if we are interested in the long-range patterns mainly. The only thing we have to take care of is that the underlying system is in the universality class of the SM. This in particular concerns the observable degrees of freedom and the emergent symmetries at work, which require the particles to be grouped predominantly in the simplest (lowest dimensional) representation of the corresponding symmetry groups. The simplest symmetry groups with singlets, doublets, and triplets are the most natural ones to emerge, as realized within the SM's $U(1)_{Y} \otimes S U(2)_{L} \otimes S U(3)_{c}$ 
gauge symmetry pattern [101-106]. More on how the SM may emerge the reader may find in the Appendix.

\section{The Hierarchy Problem revisited}

In [107] already, I outlined the flaws I see in the common reasoning concerning the hierarchy issue. As argued above, I am addressing the hierarchy problem within the LEESM "extension" of the SM. Specifically, I have in mind an implementation of the SM on a Planck lattice (see e.g. [108]). The only important point is that we can perform a low energy expansion in the corresponding cutoff. It is an accepted fact that the SM predicts a huge gap between the renormalized and the bare Higgs boson mass. From the LEESM point of view, this prediction is what promotes the Higgs boson to be a promising candidate for the inflaton. The hierarchy gap showing up is not something we have to avoid. Now, would-be infinities are replaced by eventually very large but finite numbers, and I will show that sometimes such huge effects provide what we need to understand established phenomena like inflation.

One thing we should remind here: the bare suitably regularized theory has always been the true one. Renormalization always has just been a reparametrization. The bare theory assumed to exhibit a cutoff of some sort, shows a cutoff dependent large-cutoff tail, sometimes called "preasymptote" $[1,92]$, which is equivalent to a renormalized local QFT in the universality class of the cutoff-system. Thereby it is not important that the bare cutoff system exhibits all symmetries the long-range tail will have because most of the symmetries of the LEET are emergent. In fact, by a reparametrization of parameters and fields of the preasymptotic theory (renormalizable tail) the residual cutoff-dependence is completely removable (see [92] and references therein). Because the renormalized tail has lost all information about the cutoff, it is nonsensical to say that in the LEET we would naturally expect the Higgs mass to be of the order of the cutoff.

However, in the LEESM "extension" of the SM, bare parameters turn into physical parameters of the underlying cutoff-system being the "true world" at short distances. Then the hierarchy problem is the problem of "tuning to criticality", which concerns the $\operatorname{dim}<4$ relevant operators, in particular the mass terms. In the symmetric phase of the SM, there is only one mass to be renormalized, the others being forbidden by the known chiral and gauge symmetries. For the Higgs field mass which appears in the Higgs potential the fine-tuning to criticality has the familiar form

$$
\begin{aligned}
m_{0}^{2}\left(\mu_{1}=M_{\mathrm{Pl}}\right) & =m^{2}\left(\mu_{2}=M_{H}\right)+\delta m^{2}\left(\mu_{1}, \mu_{2}\right) ; \\
\delta m^{2} & =\frac{\Lambda_{\mathrm{Pl}}^{2}}{16 \pi^{2}} C(\mu),
\end{aligned}
$$

with a coefficient typically $C=O(1)$. To keep the renormalized mass at some small value, which can be seen at low energy, formally $m_{0}^{2}$ has to be adjusted to compensate the huge number $\delta m^{2}$ revealed by the perturbative SM calculation such that about 35 digits must be adjusted in order to get the observed value below the electroweak scale. Is this a problem? 
One thing is obvious: our fine-tuning relation exhibits quantities (in the LEESM all observable in principle) at very different scales, the renormalized ones at low energy and the bare ones when approaching the Planck scale. As long as we have no direct access to the Planck physics there is no proven conflict.

Actually, a closer look reveals that in the Higgs phase there is no hierarchy problem in the SM! Why? It is true that in the relation (16) both $m_{0}^{2}$ and $\delta m^{2}$ formally may be expected many many orders of magnitude larger than $m^{2}$. Even worse, in the broken phase $\delta m^{2}$ has a huge negative value and hence $m_{0}^{2}$ must be tuned to be huge negative as well. However, in the broken phase, $m^{2} \propto v^{2}\left(\mu_{0}\right)$ is $O\left(v^{2}\right)$ not $O\left(M_{\mathrm{Pl}}^{2}\right)$. Since $v$ is the result of spontaneous symmetry breaking (non-symmetric ground state) it is per se a low energy parameter related to the emergence of long-range order. Thus in the broken phase, the Higgs boson is expected to be natural light. That the Higgs mass likely is $O\left(M_{\mathrm{Pl}}\right)$ in the symmetric phase is what realistic inflation scenarios are demanding.

In the broken phase, characterized by the non-vanishing Higgs field VEV $v(\mu) \neq 0$, all the masses are determined by the well-known mass coupling relations

$$
\begin{array}{ll}
m_{W}^{2}\left(\mu^{2}\right)=\frac{1}{4} g_{2}^{2}\left(\mu^{2}\right) v^{2}\left(\mu^{2}\right) ; & m_{Z}^{2}\left(\mu^{2}\right)=\frac{1}{4}\left(g_{2}^{2}\left(\mu^{2}\right)+g_{1}^{2}\left(\mu^{2}\right)\right) v^{2}\left(\mu^{2}\right) ; \\
m_{f}^{2}\left(\mu^{2}\right)=\frac{1}{2} y_{f}^{2}\left(\mu^{2}\right) v^{2}\left(\mu^{2}\right) ; \quad m_{H}^{2}\left(\mu^{2}\right)=\frac{1}{3} \lambda\left(\mu^{2}\right) v^{2}\left(\mu^{2}\right) .
\end{array}
$$

Here we consider the parameters in the $\overline{\mathrm{MS}}$ renormalization scheme, $\mu$ is the $\overline{\mathrm{MS}}$ renormalization scale, which we have to identify with the energy scale of the physical processes or equivalently with the corresponding temperature in the evolution of the universe. The RG equation for $v^{2}\left(\mu^{2}\right)$ follows from the RG equations for masses and massless coupling constants using one of these relations. The evolution of the $\overline{\mathrm{MS}}$ versions of $m$ and $v$ are shown in Fig. 5. As a key relation we use [68]

$$
\mu^{2} \frac{d}{d \mu^{2}} v^{2}\left(\mu^{2}\right)=3 \mu^{2} \frac{d}{d \mu^{2}}\left[\frac{m_{H}^{2}\left(\mu^{2}\right)}{\lambda\left(\mu^{2}\right)}\right] \equiv v^{2}\left(\mu^{2}\right)\left[\gamma_{m^{2}}-\frac{\beta_{\lambda}}{\lambda}\right],
$$

where $\gamma_{m^{2}} \equiv \mu^{2} \frac{d}{d \mu^{2}} \ln m^{2}$ and $\beta_{\lambda} \equiv \mu^{2} \frac{d}{d \mu^{2}} \lambda$. We write the Higgs potential as $V=\frac{m^{2}}{2} H^{2}+$ $\frac{\lambda}{24} H^{4}$, which fixes our normalization of the Higgs self-coupling. When the $m^{2}$-term changes sign and $\lambda$ stays positive, we know we have a first order phase transition (see below). Funny enough, the Higgs particle gets its mass from its interaction with its own condensate! and thus gets a mass in the same way and in the same ballpark as the heavier SM species, which couple strongest to the Higgs field. As mentioned before the Higgs mass cannot be much heavier than the other heavier particles if renormalizability is to be effective at low and moderate energies. The interrelations (17) also show that for fixed $v$, as determined by the Fermi constant $G_{\mu}=1 /\left(\sqrt{2} v^{2}\right)$, the Higgs cannot get too heavy if perturbation theory should remain applicable. Also note that the conspiracy between those couplings relevant to stabilize the vacuum only can work if these couplings are of comparable size.

Often an extreme point of view is taken: all particles naturally should have masses $O\left(M_{\mathrm{Pl}}\right)$ i.e. $v=O\left(M_{\mathrm{Pl}}\right)$. This would mean that the symmetry is not recovered at the high (=bare) scale and the notion of spontaneous symmetry breaking would be obsolete! Of course, this makes no sense. In a perturbative calculation within a cutoff 

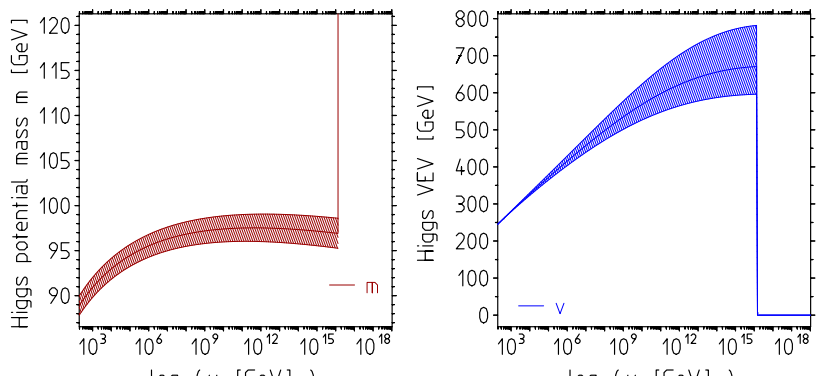

$\log (\mu[\mathrm{GeV}])$

$\log (\mu[\mathrm{GeV}])$

Figure 5: Dimensionful SM running $\overline{\mathrm{MS}}$ parameters $m$ and $v=\sqrt{6 / \lambda} m$. Error bands include SM parameter uncertainties and a Higgs boson mass range $125.5 \pm 1.5 \mathrm{GeV}$ which essentially determines the widths of the bands

regulated theory formally one finds $v=O\left(M_{\mathrm{Pl}}\right)$ but in the broken phase $\delta m_{H}^{2}$ is huge negative, which requires a non-perturbative vacuum rearrangement revealing the mentioned mass coupling relations in terms of a renormalized effective $v$ also for the Higgs particle. Since $v \equiv 0$ above the EW phase-transition point, it makes no sense to say that one naturally has to expect $v\left(\mu=M_{\mathrm{Pl}}\right)=O\left(M_{\mathrm{Pl}}\right)$. The Higgs-field VEV $v$ is an order parameter, related to the spontaneous breaking of the discrete symmetry ${ }^{14} Z_{2}: H \leftrightarrow-H$, and is resulting from long range collective behavior. It can be as small as we like. Its value is a function of the effective temperature (energy scale) with its maximum at $T=0$, monotonically decreasing with increasing temperature and vanishing at the second order phase transition point $T_{c}$ above which $v(T) \equiv 0$ vanishes identically (nonanalyticity).

A well known prototype for long range order is the magnetization in a ferromagnetic spin system ${ }^{15}$ illustrated in Fig. 6.

The analogy shows us that $v / M_{\mathrm{Pl}} \ll 1$ is not unnatural since $v \neq 0$ emerges only below a critical temperature, which is not in a simple way related to $M_{\mathrm{Pl}}$. The EW scale is set by $v(\mu)$ and depends on $\mu$. As we learn from Fig. 5, at low energy $v(0)=$ $1 /\left(\sqrt{2} G_{\mu}\right)^{1 / 2} \approx 246 \mathrm{GeV}$, but interestingly, in contrast to the magnetization of a ferromagnet, $v(\mu)$ is increasing rather than monotonically decreasing with increasing $\mu$.

\footnotetext{
${ }^{14}$ In the unitary gauge, we can avoid problems related to Elitzur's theorem [109], which claims that an order parameter cannot be associated with SSB of a non-Abelian gauge theory. In a physical gauge, on physical Hilbert space, Higgs ghost fields are absent and a Mexican hat potential is a phantom as it only exist if ghost space is taken into the display. A physical Mexican hat potential would imply the existence of three Nambu-Goldstone bosons.

${ }^{15}$ As an example we may consider an Ising ferromagnet in $D=2$ dimensions, $J$ is the nearest neighbor (n.n.) spin coupling between the spins on a lattice

$$
H(\sigma)=-J \sum_{<i j>} \sigma_{i} \sigma_{j} ; P_{\beta}(\sigma)=\frac{\mathrm{e}^{-\beta H(\sigma)}}{Z_{\beta}} ; Z_{\beta}=\sum_{\sigma} \mathrm{e}^{-\beta H(\sigma)} .
$$

Here $\beta=\frac{1}{k_{B} T}$ where $k_{B}$ is the Boltzmann constant. The Onsager solution for the critical temperature reads $\sinh ^{2}\left(\frac{2 J}{k_{B} T}\right)=1 ; T_{c}=\frac{2 J}{k_{B} \ln (1+\sqrt{2})}$ and the magnetization is given by $M=\left(1-[\sinh 2 \beta J]^{-4}\right)^{\frac{1}{8}}$, depending on temperature $T$ and n.n. spin interaction strength $J$. For more details see e.g. [91]
} 


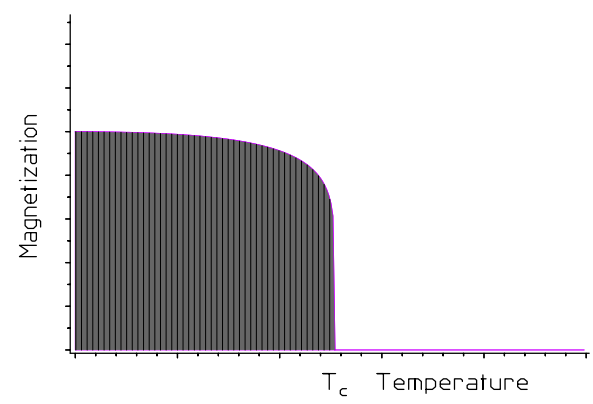

Figure 6: Spontaneous magnetization $M=M(T)$ as a function of temperature $T . T_{c}$ is the critical temperature above which $M(T) \equiv 0$ for all $T>T_{c}$. Furthermore, $M(T) \rightarrow 0$ as $T \leftrightharpoons T_{c}$ may be as small as we like depending on the distance $T-T_{c}$ from criticality. Note that $M(0)$ is not given by what would correspond to the cutoff of the ferromagnetic system, even if it would be measured in units of the cutoff

This is because of the rich conspiring dynamics of the SM encoded in the RG equation $(18)^{16}$, yet $v(\mu)$ is vanishing at $\mu_{0} \sim 10^{16} \mathrm{GeV}: v(\mu) \rightarrow 0$ when $\mu \leftrightharpoons \mu_{0}$, as we will see later. The second order phase transition (PT) point is a point of non-analyticity i.e. exhibits singular behavior and physics in the ordered phase and the disordered phase are very different.

Considering a ferromagnet one has to tune the temperature $T$ to criticality in order to find the PT point. What is tuning the temperature to criticality in the SM? The answer is the expansion of the universe, which provides a scan in temperature (see also [110]). The maximum value of $v(\mu)$ is achieved in the low energy limit at $\mu=0$. Why should the magnitude of $v(0)$ be of the order of the Planck scale, given the fact that above the phase transition point, in the disordered phase, the VEV is actually vanishing identically?

This shows that the Higgs boson mass renormalization equation is not a static equation but is subject to a sophisticated dynamics driven by the expansion of the universe.

In the symmetric phase at very high energy, we see the bare system. There the Higgs field is a collective field exhibiting an effective mass generated by radiative effects within the Planck system such that $m_{0}^{2} \approx \delta m^{2}$ at $M_{\mathrm{Pl}}$. In particle physics, a radiatively induced mass is known from the Coleman-Weinberg mechanism [111], now in the symmetric phase and applied to the Planck medium. Such a mechanism, which is natural in this context, eliminates a possible fine-tuning problem at all scales. There are many examples in condensed matter systems, like the effective mass of the photon in the superconducting phase (Meissner effect) or the effective mass of the effective field which encodes the spin-singlet electron pairs (Cooper pairs) in the GinzburgLandau (GL) model [112] of superconductivity ${ }^{17}$. The latter directly corresponds to

\footnotetext{
${ }^{16}$ Fig. 5 shows that the Higgs mass parameter $m$ is varying little in the broken phase, while $v=\sqrt{6 / \lambda} m$ increases substantially because $\lambda$ decreases rapidly.

${ }^{17}$ Originally the Ginzburg-Landau theory of superconductivity has been proposed as a macroscopic phenomenological effective theory describing type-I superconductors without reference to microscopic properties. Later Bardeen-Cooper-Schrieffer could explain superconductivity from its microscopic structure in
} 
the Abelian Higgs model. Emerging as an effective field from the hot Planck system, which exhibits all types of excitations, it is also pretty obvious that the Higgs field couples to all these modes that we see as Yukawa and Higgs to gauge boson couplings in the SM. That these couplings exhibit the symmetries of the SM is again due to the fact that only the renormalizable tail can be seen at low energies. All Planck system modes that do not conspire, as SM degrees of freedom and their couplings do within the SM, are not perceivable at long distances. The SM emerges as a self-organized system.

On the one hand, we know that astronomy and astrophysics are unthinkable without the input from laboratory physics in general and particle physics in particular. On the other hand, it is not new that particle physics is learning from cosmology. What is required to explain inflation, baryogenesis, nucleosynthesis, CMB patterns, dark matter, etc. ? If the SM has an extrapolation up to the Planck scale, evidently one is able to confront SM predictions with physics established to have happened in the early universe. In contrast to the old paradigm of an empty vacuum: we know that the ground state of the world is filled with dark energy, with a Higgs condensate and quark and gluon condensates. All these effects have been showing up at certain times and play a key role in the evolution of the universe. Obviously, there are plenty of questions to be answered in order to get a better understanding of how the universe has been shaped after the Big Bang.

\section{Running SM parameters trigger the Higgs mecha- nism}

In Sect. 2.2 already, we have discussed how the Higgs boson discovery has been revealing a peculiar value for the Higgs boson self-coupling, which largely clarified the path of extrapolating the SM to higher energies. We remind that all dimensionless couplings satisfy the same RG equations in the broken and in the unbroken phase and are not affected by any power cutoff dependencies. This is as it has to be because the Higgs mechanism (SSB) does not alter the UV behavior. The evolution of the SM couplings in the $\overline{\mathrm{MS}}$ scheme up to the Planck scale has been investigated in [50,58-65,70,116,117], and has been extended to include the Higgs-field VEV and the masses in [1,69]. Except for $g_{1}$, which increases very moderately, all other couplings decrease and stay positive up to the Planck scale. This strengthens the reliability of perturbative arguments and reveals a stable Higgs potential up to the Planck scale $[1,69]$. While most analyses $[58,61,62,70,116,117]$ find that for the given Higgs mass value range vacuum stability is nearby only (meta-stability) ${ }^{18}$, and the SM actually fails to persist up to the

their BCS-theory [113]. Afterward, Gor'kov derived the GL-theory [114] showing that in some limit all GL parameters have a microscopic interpretation. In addition, Abrikosov showed that GL-theory also models type-II superconductors [115]. The effective GL-theory thus efficiently describes a rich variety if superconducting systems, without the need for a detailed microscopic understanding.

${ }^{18}$ Most groups are adopting essentially the same input parameters presented in $[58,61,70]$ and a radiatively corrected effective potential and find the vacuum to lose stability at about a surprisingly low scale of about $\mu \sim 10^{9} \mathrm{GeV}$ [input not independent]. Keep in mind: the Higgs boson mass miraculously turns out to have a value very close to what was expected from vacuum stability. It looks like a tricky conspiracy with other couplings to reach this "purpose". Assuming vacuum stability, the narrow stability window actually makes the Higgs mass to be a predictable quantity if we consider the other SM parameters as given. Also imposing 
Table 1: Comparison of $\overline{\mathrm{MS}}$ parameters at various scales: Running couplings for $M_{H}=$ $126 \mathrm{GeV}$ and $\mu_{0} \simeq 1.4 \times 10^{16} \mathrm{GeV}$. Note that $\lambda=0$ is an essential singularity and the theory cannot be extended beyond a possible zero of $\lambda$. Remind that $v=\sqrt{6 m^{2} / \lambda}$ i.e. $v(\lambda) \rightarrow \infty$ as $\lambda \rightarrow 0$. Besides the Higgs boson mass $m_{H}=\sqrt{2} m$ all masses $m_{i} \propto g_{i} v \rightarrow \infty$ would yield a different cosmology

\begin{tabular}{ccccc||cc}
\hline \hline & \multicolumn{3}{c}{ my findings [Jeg] } & \multicolumn{2}{c}{ Degrassi et al. 2013 [Deg] } \\
\hline coupling \scale & $M_{Z}$ & $M_{t}$ & $\mu_{0}$ & $M_{\mathrm{Pl}}$ & $M_{t}$ & $M_{\mathrm{Pl}}$ \\
\hline$g_{3}$ & 1.2200 & 1.1644 & 0.5271 & 0.4886 & 1.1644 & 0.4873 \\
$g_{2}$ & 0.6530 & 0.6496 & 0.5249 & 0.5068 & 0.6483 & 0.5057 \\
$g_{1}$ & 0.3497 & 0.3509 & 0.4333 & 0.4589 & 0.3587 & 0.4777 \\
$y_{t}$ & 0.9347 & 0.9002 & 0.3872 & 0.3510 & 0.9399 & 0.3823 \\
$\sqrt{\lambda}$ & 0.8983 & 0.8586 & 0.3732 & 0.3749 & 0.8733 & $\mathrm{i} 0.1131$ \\
$\lambda$ & 0.8070 & 0.7373 & 0.1393 & 0.1405 & 0.7626 & -0.0128 \\
\hline
\end{tabular}

Planck scale, our evaluation of the matching conditions yields initial $\overline{\mathrm{MS}}$ parameters at the $Z$ boson mass scale which evolve preserving the positivity of $\lambda$. Thereby the critical parameter is the top-quark Yukawa coupling, for which we find a slightly lower value, which is based on the analysis [69]. My MS input at $M_{Z}$ is [1] $g_{3}=1.2200$, $g_{2}=0.6530, g_{1}=0.3497, y_{t}=0.9347$ and $\lambda=0.8070$. At $M_{\mathrm{Pl}} \mathrm{I}$ get $g_{3}=0.4886$, $g_{2}=0.5068, g_{1}=0.4589, y_{t}=0.3510$ and $\lambda=0.1405$ (see Table 1). In view of the fact that the precise meaning of the experimentally extracted value of the top-quark mass is not free of ambiguities, usually, it is identified with the on-shell mass $M_{t}$ (see e.g. $[62,69,118]$ and references therein), it may be premature to claim that instability of the SM Higgs potential is a proven fact already [71]. As I have elaborated in Sect. 2.1, the implementation of the matching conditions is not free of ambiguities, while the evolution of the couplings over many orders of magnitude is rather sensitive to the precise values of the initial couplings. Accordingly, all numbers presented in this article depend on the specific input parameters adopted, as specified in [1,69]. In case the Higgs self-coupling has a zero $\lambda\left(\mu^{2}\right)=0$, at some critical scale $\mu_{c}$ below $M_{\mathrm{Pl}}$, we learn from Eq. (18), or more directly from $v\left(\mu^{2}\right)=\sqrt{6 m^{2}\left(\mu^{2}\right) / \lambda\left(\mu^{2}\right)} \stackrel{\lambda \rightarrow+0}{\rightarrow} \infty$ that the SM loses its "being well-defined" above this singular non-analytic point ${ }^{19}$.

For our input parameters, Table 1 shows that the relevant running $\overline{\mathrm{MS}}$ parameters at the Planck scale are of comparable size in the range 0.51 for $g_{2}$ being the largest here and 0.35 for $y_{t}$ being the smallest, with $\sqrt{\lambda}$ at 0.375 slightly larger in our normalization. It tells us that approximations like the gaugeless limit $\left(g_{1}=g_{2}=0\right)$ or assuming $\lambda \approx 0$

Planck-scale boundary conditions may be argued to fix the Higgs boson mass [59,60]. If the Higgs boson misses to stabilize the vacuum, why does it just miss it almost not?

${ }^{19}$ As we have argued earlier we consider the bare Higgs potential to be the true potential, except that the bare parameters have to be calculated bottom-up from the known values at low energy. A low energy reparametrization also affects the form of the potential by radiative corrections as we know from ColemanWeinberg [111]. The correspondingly modified effective potential plays a crucial role when the potential gets unstable and actually can turn instability into meta-stability [37,58]. This will be discussed in Sect. 5.1 below. The Planck medium, from which the SM derives as a long distance tail, certainly exhibits a stable ground state. This we infer from our mere existence. 
relative to other couplings are not viable approximations near $M_{\mathrm{Pl}}$.

For what follows we take up what Shaposhnikov et al. [37] say about vacuum stability in their conclusion: "Although the present experimental data are perfectly consistent with the absolute stability of the Standard Model within the experimental and theoretical uncertainties, one should not exclude the possibility that other experiments will be able to establish the meta-stability of the electroweak vacuum in the future." But, based on a slightly modified evaluation of $\overline{\mathrm{MS}}$ parameters [69] (which revealed vacuum stability), we adopt the view: "Although other evaluations of the matching conditions seem to favor the meta-stability of the electroweak vacuum within the experimental and theoretical uncertainties, one should not exclude the possibility that other experiments and improved matching conditions will be able to establish the absolute stability of the Standard Model in the future."

Running couplings can affect dramatically the quadratic divergences and the interpretation of the hierarchy problem. Quadratic divergences have been investigated at one-loop in [4] (see also $[5,119,120]$ ), at two loops in [6-8]. At $n$ loops the quadratic cutoff-dependence is of the form

$$
\delta m_{H}^{2}=\frac{\Lambda^{2}}{16 \pi^{2}} C_{n}(\mu),
$$

where the n-loop coefficient only depends on the gauge couplings $g_{1}, g_{2}, g_{3}$, the Yukawa couplings $y_{f}$ and the Higgs self-coupling $\lambda$. Neglecting the numerically insignificant light fermion contributions, the one-loop coefficient function $C_{1}$ may be written as

$$
C_{1}=2 \lambda+\frac{3}{2} g_{1}^{2}+\frac{9}{2} g_{2}^{2}-12 y_{t}^{2}
$$

and is uniquely determined by dimensionless couplings. The latter are not affected by quadratic divergences such that standard RG equations apply. Surprisingly, as first pointed out by Hamada, Kawai and Oda in [7], taking into account the running of the SM couplings, the coefficient of the quadratic divergences of the bare Higgs mass correction can vanish at some scale, given the specific SM couplings that became available after the Higgs boson discovery. In contrast to our evaluation Hamada et al. actually find the zero to lie above the Planck scale, having adopted input $\overline{\mathrm{MS}}$ parameters from [58]. In our analysis, relying on matching conditions for the top-quark mass analyzed in [69], we get a scenario where $\lambda\left(\mu^{2}\right)$ stays positive up to the Planck scale and looking at the relation between the bare and the renormalized Higgs mass we find $C_{1}$ and hence the Higgs mass counterterm to vanish at about $\mu_{0} \sim 1.4 \times 10^{16} \mathrm{GeV}$, not very far below the Planck scale. The next-order correction, first calculated in $[6,8]$ and confirmed in [7] reads

$$
\begin{aligned}
C_{2}= & C_{1}+\frac{\ln \left(2^{6} / 3^{3}\right)}{16 \pi^{2}}\left[18 y_{t}^{4}+y_{t}^{2}\left(-\frac{7}{6} g_{1}^{2}+\frac{9}{2} g_{2}^{2}-32 g_{3}^{2}\right)\right. \\
& \left.-\frac{87}{8} g_{1}^{4}-\frac{63}{8} g_{2}^{4}-\frac{15}{4} g_{2}^{2} g_{1}^{2}+\lambda\left(-6 y_{t}^{2}+g_{1}^{2}+3 g_{2}^{2}\right)-\frac{2}{3} \lambda^{2}\right],
\end{aligned}
$$

and numerically does not change the one-loop result significantly. The same results apply for the Higgs potential parameter $m^{2}$, which corresponds to $m^{2} \hat{=} \frac{1}{2} m_{H}^{2}$ in the broken 

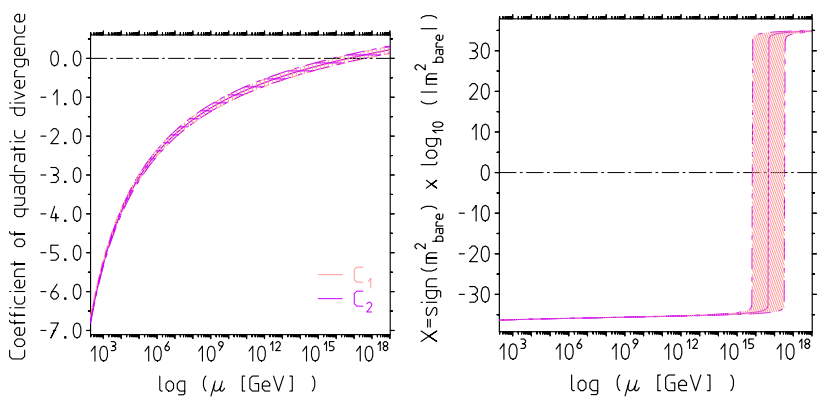

Figure 7: The Higgs mechanism transition in the SM. Left: the zero in $C_{1}$ and $C_{2}$ for $M_{H}=125.9 \pm 0.4 \mathrm{GeV}$. Right: shown is $X=\operatorname{sign}\left(m_{\text {bare }}^{2}\right) \times \log _{10}\left(\left|m_{\text {bare }}^{2}\right|\right)$, which represents $m_{\text {bare }}^{2}=\operatorname{sign}\left(m_{\text {bare }}^{2}\right) \times 10^{X}$

phase. For scales $\mu<\mu_{0}$ we have $\delta m^{2}$ large negative, which is triggering spontaneous symmetry breaking by a negative bare mass $m_{0}^{2}=m^{2}+\delta m^{2}$, where $m$ again denotes the renormalized mass. The phase transition is illustrated in Fig. 7. The jump taking place here in the vacuum energy is given by ${ }^{20}$

$$
\Delta V\left(\phi_{0}\right)=-\frac{m_{\mathrm{eff}}^{2} v^{2}}{8}=-\frac{\lambda v^{4}}{24} \sim-9.6 \times 10^{8} \mathrm{GeV}^{4} \approx-(176.0 \mathrm{GeV})^{4} .
$$

As a CC contribution it is of negative sign and 50 orders of magnitude off relative to what corresponds to the observed $\Lambda_{\mathrm{CMB}}$ (see also [22]). However, the effect is small relative to the $O\left(M_{\mathrm{Pl}}^{4}\right)$ size $V(0)=\langle V(\phi)\rangle$, which will be discussed in Sect. 6. At $\mu=\mu_{0}$ we have $\delta m^{2}=0$ and the sign of $\delta m^{2}$ flips, implying a phase transition to the symmetric phase. Finite temperature effects [121-124], which must be included in a realistic scenario, turn out not to change the gross features of our scenario, unless $\mu_{0}$ would turn out to lie much closer to $\Lambda_{\mathrm{Pl}}$ [1]. A different effect is due to the change in the effective mass resulting from the Wick reordering of the Lagrangian by a nonvanishing $\left\langle\Phi^{+} \Phi\right\rangle$. This will be discussed in Sect. 6. It produces a larger shift of the transition point as one may learn from Fig. 8, where the finite temperature effects are displayed. What do we learn from this analysis? The Higgs mechanism is dynamically triggered as the temperature in the universe drops below $\mu_{0}$. In the low energy phase, the Higgs boson mass $M_{H}$ substitutes $\sqrt{2} m$ and in fact has to be calculated from the vacuum rearrangement (see Fig. 12). Now $m_{H}$ turns into an emergent mass, which is determined by the mass-coupling relation (17) like for all other massive particles in the Higgs phase. At the transition point, we have $\delta m^{2}=0$ and no hierarchy problem. While above $\mu_{0}$ the shift $\delta m^{2}$ is physical and emergent from the interaction in the Planck medium, below $\mu_{0}$, the shift $\delta m^{2}$ looses its physical meaning. This is because at $\mu=\mu_{0}$ the enhanced cutoff-effects are nullified. At this point, the access to cutoff effects gets lost and we enter the renormalizable renormalized low energy phase. Below $\mu_{0}$, we still use $\delta m_{H}^{2}$ in perturbative mass renormalization, where it is now large negative if

\footnotetext{
${ }^{20}$ Note that this is a finite prediction independent of quadratic cutoff effects. The transition point $\mu_{0}$ is a matching point where bare and renormalized quantities at scale $\mu_{0}$ agree, i.e. $\lambda=\lambda\left(\mu_{0}\right)$ and $v=v\left(\mu_{0}\right)$.
} 

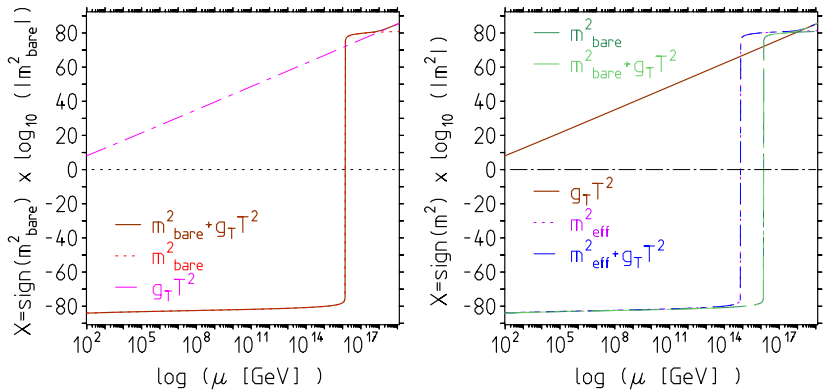

Figure 8: $X$ as displayed in the right panel of Fig. 7 including leading finite temperature correction to the potential $V(\phi, T)=\frac{1}{2}\left(g_{T} T^{2}+m_{0}^{2}\right) \phi^{2}+\frac{\lambda}{24} \phi^{4}+\cdots$ with $g_{T}=$ $\frac{1}{16}\left[3 g_{2}^{2}+g_{1}^{2}+4 y_{t}^{2}+\frac{2}{3} \lambda\right]$ from [125] affecting the phase transition point. Left: for the bare case $\left[\mathrm{m}^{2}, C_{1}\right]$. Right: with adjusted effective mass from vacuum rearrangement $\left[\mathrm{m}^{\prime 2}, C_{1}^{\prime}=C_{1}+\lambda\right]$. In the case $\mu_{0}$ sufficiently below $M_{\mathrm{Pl}}$, the case displayed here, finite temperature effects affect the position of the phase transition little, while the change of the effective mass by the vacuum rearrangement is more efficient. The finite temperature effect with our parameters is barely visible

we still insist on using the now physics-wise inaccessible Planck scale as a UV cutoff. I would say that argumentations based on (16) now turn into formal nonsense. Not only the magnitude of the cancellation is arbitrary, but it also has the wrong sign, for what could be related to a physical mass. The physical mass is determined by the curvature at the minimum of the potential. The key outcome of our calculation is the observation that the SM at high enough sub-Planckian energies undergoes a transition into the symmetric phase [1], presuming a stable vacuum. Fig. 1 displays the SM prediction for the effective Higgs mass as a function of the energy scale. This profile promotes the Higgs boson to act as an inflaton as discussed in Sect. 1 already.

\subsection{Vacuum stability and effective potential}

The classical Higgs potential (2) for $\lambda>0$ is bounded from below and has a trivial minimum for $m^{2}>0$ at $\phi_{0}=0$, and a non-trivial minimum at $\phi_{0}^{2}=\frac{-6 m^{2}}{\lambda}$ for $m^{2}<0$. When the classic potential turns unstable, because $\lambda$ is running to be negative, the analysis of the vacuum stability has to be based on the effective potential, which is 
obtained by including the quantum corrections $[111,126]^{21}$. The effective potential is gauge- and scale-dependent and not an observable. In the Landau gauge and the $\overline{\mathrm{MS}}$ scheme it can be written as [127-130] (also see [51]) ${ }^{22}$

$$
V_{\mathrm{eff}}(\phi(t))=\frac{1}{2} m^{2}(t) \phi^{2}(t)+\frac{1}{24} \lambda(t) \phi^{4}(t)+V_{1}+V_{2}+V_{3}+V_{\mathrm{rem}},
$$

with

$$
\begin{aligned}
V_{1}=\kappa & \left\{\frac{3}{2} m_{W}^{4}(t)\left[\ln \frac{m_{W}^{2}(t)}{\mu^{2}(t)}-\frac{5}{6}\right]+\frac{3}{4} m_{Z}^{4}(t)\left[\ln \frac{m_{Z}^{2}(t)}{\mu^{2}(t)}-\frac{5}{6}\right]-3 m_{t}^{4}(t)\left[\ln \frac{m_{t}^{2}(t)}{\mu^{2}(t)}-\frac{3}{2}\right]\right. \\
& \left.+\frac{1}{4} m_{H}^{4}(t)\left[\ln \frac{m_{H}^{2}(t)}{\mu^{2}(t)}-\frac{3}{2}\right]+\frac{3}{4} m_{G}^{4}(t)\left[\ln \frac{m_{G}^{2}(t)}{\mu^{2}(t)}-\frac{3}{2}\right]\right\}
\end{aligned}
$$

where $\kappa=1 /(4 \pi)^{2}$ and $m_{i}$ are the masses of different particles in the background of the classical Higgs source field $\phi_{c}$ of the generating functional for the irreducible Higgs vertex functions, which upon renormalization is given by $\phi(t)=Z_{\phi}(t) \phi_{c}$. Thus we have

$$
\begin{aligned}
& m_{W}^{2}(t)=\frac{1}{4} g_{2}^{2}(t) \phi^{2}(t), m_{Z}^{2}(t)=\frac{1}{4}\left[g_{2}^{2}(t)+g_{1}^{2}(t)\right] \phi^{2}(t), m_{t}^{2}(t)=\frac{1}{2} y_{t}^{2}(t) \phi^{2}(t), \\
& m_{H}^{2}(t)=m^{2}+\frac{1}{2} \lambda \phi^{2}(t), m_{G}^{2}(t)=m^{2}+\frac{1}{6} \lambda \phi^{2}(t) .
\end{aligned}
$$

The effective potential as derived in the symmetric phase include the would-be Higgs ghosts $G$ contribution as physical degrees of freedom, in the broken phase Higgs ghosts are massless in the Landau gauge (would-be Nambu-Goldstone bosons). In the symmetric phase they contribute as three additional Higgs particles. As we know the Higgs boson mass in the broken phase $\left(m^{2}<0\right)$ is $M_{H}^{2}=-2 m^{2}=\frac{1}{3} \lambda v^{2}$, where $v$ refers to the EW vacuum. Two-loop corrections $V_{2}$ have been calculated in [128, 129] and may

\footnotetext{
${ }^{21}$ Being a part of the SM Lagrangian the Higgs potential term considered so far gets reparametrized by a change of the effective parameters and the effective Higgs field and by appropriate counterterms only, as long as perturbation theory does not break down. All perturbative physics is obtained as usual by means of the renormalizable Lagrangian, written in terms of the quantized fields, and the corresponding Feynman rules. Also note that the Higgs contribution to the energy-momentum tensor of Einstein gravity is represented by the symmetric energy-momentum tensor

$$
\Theta^{\mu}{ }_{v}=\frac{\partial \mathcal{L}}{\partial\left(\partial_{\mu} \phi\right)} \partial_{\nu} \phi-\delta^{\mu} \mathcal{L}, \text { where } \mathcal{L}(\phi)=\frac{1}{2} g^{\mu v} \partial_{\mu} \phi \partial_{\nu} \phi-V(\phi),
$$

in terms of the Higgs part of the bare SM Lagrangian.

${ }^{22}$ As shown in [111], the potential satisfies the RG equation

$$
\left(\mu \frac{\partial}{\partial \mu}+\sum_{i} \beta_{i} \frac{\partial}{\partial \lambda_{i}}+\gamma \phi \frac{\partial}{\partial \phi}\right) V=0
$$

where $\lambda_{i}=m^{2}, \lambda, g^{\prime}=g_{1}, g=g_{2}, g_{s}=g_{3}, y_{t}$ with corresponding beta-functions $\beta_{i}$ and $\gamma$ the anomalous dimension of the Higgs field. The RG as usual is solved along characteristic curves where $t$ parametrizes the position on the curve. The solution reads

$$
V\left(\mu, \lambda_{i}, \phi\right)=Z_{\phi}^{4}(t) V\left(\mu(t), \lambda_{i}(t), \phi\right),
$$

with $Z_{\phi}(0)=1, \lambda_{i}=\lambda_{i}(0)$ and $\phi=\phi(0)$.
} 
be found in more condensed form in [58]. $V_{3}$ includes the leading three-loop corrections computed in [131]. The remainder $V_{\text {rem }}$ represents the higher-order contributions, which include also the higher dimension operators starting at four loops [129,132,133]:

$$
V_{\text {rem }} \sim \lambda \phi^{4} \sum_{L>4}\left(\frac{\lambda^{2}}{M_{P l}^{2}} \phi^{2}\right)^{L-3}
$$

where $L$ is number of loops.

The wavefunction renormalization of the Higgs field takes the form

$$
\phi(t)=Z_{\phi}(t) \phi_{c}=\exp \left\{\int_{0}^{t} \gamma(\tau) \mathrm{d} \tau\right\} \phi(0), \phi(0)=\phi_{c},
$$

where $\gamma(t)=\mathrm{d} \ln Z_{\phi} / \mathrm{d} t$ is the anomalous dimension of the Higgs field:

$$
\begin{aligned}
\gamma= & \kappa\left[\frac{9}{4} g_{2}^{2}+\frac{3}{4} g_{1}^{2}-3 y_{t}^{2}\right]+\kappa^{2}\left[y_{t}^{2}\left(\frac{27}{4} y_{t}^{2}-20 g_{3}^{2}-\frac{45}{8} g_{2}^{2}-\frac{85}{24} g_{1}^{2}\right)\right. \\
& \left.+\frac{271}{32} g_{2}^{4}-\frac{9}{16} g_{2}^{2} g_{1}^{2}-\frac{431}{96} g_{1}^{4}-\frac{1}{6} \lambda^{2}\right]+\cdots
\end{aligned}
$$

Finally, the scale $\mu(t)$ is related to the running parameter $t$ by

$$
\mu(t)=\mu \mathrm{e}^{t} \text {, i.e. } t=\ln \mu(t) / \mu,
$$

where $\mu$ is a fixed scale, that we will take equal to the physical top-quark mass, $M_{t}$ as a reference point. Observable physical predictions up to perturbative truncation errors do not depend on the choice of the renormalization scale. This can be used in order to keep radiative corrections moderate by choosing $\mu(t)=\phi(t)$ which avoids large logarithms at any given $t$, since then $\ln m_{W}^{2}(t) / \mu^{2}(t)=\ln g^{2}(t) / 4$ etc. (see [129]). One also may choose $\mu(t)=\phi_{c}$ in which case $\ln m_{W}^{2}(t) / \mu^{2}(t)=\ln g^{2}(t) / 4+2 \Gamma$ etc. The correction $\Gamma=\int_{M_{t}}^{\phi} \gamma(\mu) \mathrm{d} \ln (\mu)$ stems from the field renormalization factor $Z_{\phi}$.

As elaborated in [51] for high Higgs fields the effective potential may be cast into the simple form where it is dominated by the quartic term

$$
V_{\mathrm{eff}} \approx \frac{\lambda_{\mathrm{eff}}(\phi)}{24} \mathrm{e}^{4 \Gamma(\phi)} \phi_{c}^{4}
$$

and $\lambda(\phi)$ depends on $\phi$ the same as the running coupling $\lambda(\mu)$ depends on the running scale $\mu=\phi_{c}$ with modified coupling [70]

$$
\begin{aligned}
\lambda_{\mathrm{eff}} \approx \lambda+\quad \kappa & \quad\left[\frac{9}{4} g_{2}^{4}\left(\ln \frac{g_{2}^{2}}{4}-\frac{5}{6}+2 \Gamma\right)+\frac{9}{8}\left(g_{2}^{2}+g_{1}^{2}\right)^{2}\left(\ln \frac{g_{2}^{2}+g_{1}^{2}}{4}-\frac{5}{6}+2 \Gamma\right)\right. \\
& -18 y_{t}^{4}\left(\ln \frac{y_{t}^{2}}{2}-\frac{3}{2}+2 \Gamma\right)+\frac{3}{2} \lambda^{2}\left(\ln \frac{|\lambda|}{2}-\frac{3}{2}+2 \Gamma\right)+\frac{1}{2} \lambda^{2}\left(\ln \left(\frac{|\lambda|}{6}-\frac{3}{2}+2 \Gamma\right)\right] \\
+ & \kappa^{2} \quad 6 y_{t}^{4}\left[8 g_{3}^{2}\left(3 r_{t}^{2}-8 r_{t}+9\right)-\frac{3}{2} y_{t}^{2}\left(3 r_{t}^{2}-16 r_{t}+23+\frac{\pi^{2}}{3}\right)\right]+\cdots
\end{aligned}
$$



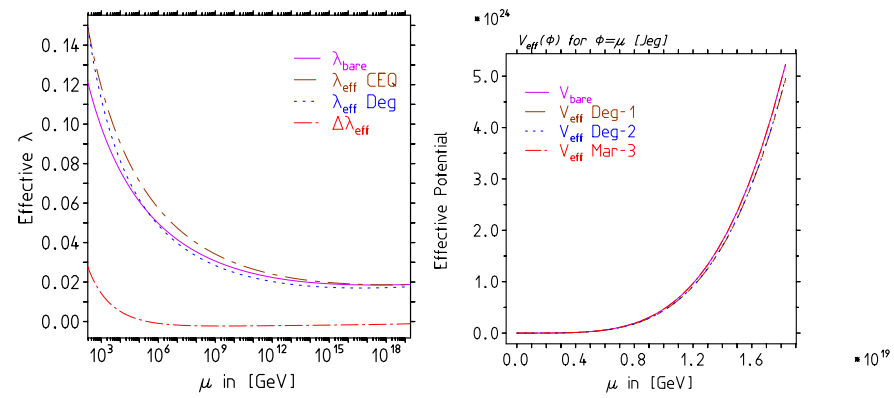

Figure 9: The bare versus the effective Higgs coupling and the effective potential for the parameter set [Jeg] of Table 1. Left: the effective Higgs self-coupling $\lambda_{\text {eff }}$ governing the SM effective potential $V_{\text {eff }} \sim \frac{\lambda_{\text {eff }}}{24} \phi^{4}$ for large fields. "CEQ" is one-loop improved from [51], "Deg" is two-loop improved from [58]. The correction $\Delta \lambda_{\text {eff }}$ represents the corrections included in "Deg" relative to $\lambda_{\text {bare }}$. Right: the bare potential compared with different approximations of the effective potential: one-loop improved "Deg-1", two-loop improved "Deg-2" and three-loop improved "Mar-3" [131].

up to less relevant corrections. The crucial point is that for parameters as [Deg] in Table 1 the correction term is positive all up to the Planck scale. At the EW scale the leading positive $\lambda$-term dominates $\lambda_{\text {eff }}$ up to scales where $\lambda$ approaches a zero and there changes the sign, if such a zero exists, which depend on the precise input values at the EW scale. In the vicinity above the zero of $\lambda$ actually $\lambda_{\text {eff }}$ remains positive and such stabilizes the Higgs vacuum to somewhat higher scales but also turns negative to a metastable state before reaching the Planck scale (see Fig. 3 in [58]). In contrast for the parameter set [Jeg] the correction $\Delta \lambda_{\text {eff }}$ also starts positive but at higher scales takes negative values. These are small, however, and are not affecting the positivity of $\lambda_{\text {eff }}$ itself as seen in the left panel of Fig. 9. In the stable vacuum scenario radiative corrections of the effective potential are moderate and do not affect the main pattern as long as $\lambda$ remains positive. The cutoff power enhanced effects are always much larger than the standard radiative corrections to the effective potential, provided the formers are taken into account. This we will have to remind also for the discussion to follow in Sect. 6. The quantum corrections modify the shape of the potential such that a second minimum at some higher (Planck) scale may be induced (see Fig. 10 and Fig. 7 in [58]). As first discussed in [129], a second minimum is also obtained when a transient instability emerges above our Higgs transition point $\mu_{0}$ when the bare mass term gets positive and actually gets huge because of the quadratic cutoff-enhancement. For especially fine-tuned parameters this may happen also if radiative corrections are not yet included. In any case, the existence of a second minimum depends significantly on the higher order corrections. Depending on the values of the Higgs boson and topquark masses the lifetime of the EW vacuum can be larger or smaller than the age of the Universe. The first case corresponds to the metastable scenario.

For stationary points $\phi_{0}$ much larger than the electroweak scale one has $\lambda_{\mathrm{eff}} \ll 1$ 


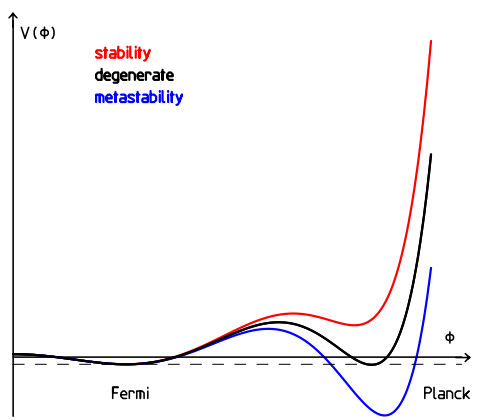

Figure 10: The form of the effective potential for the Higgs field $\phi$ which corresponds to the stable, critical and metastable electroweak vacuum. The pattern displayed, admitting for two minima at non-zero field values requires the effective potential to exhibit even powers of $\phi$ up to $\phi^{8} . v$ is the location of the EW minimum and $\phi_{\min } \gg v$ is the value of a new minimum.

and the curvature of the potential is given by [52]

$$
\left.\frac{\partial^{2} V_{\mathrm{eff}}}{\partial \phi^{2}(t)}\right|_{\phi=\phi_{0}}=\frac{1}{2}\left(\beta_{\lambda}-4 \gamma \lambda\right) \phi_{0}^{2} \approx \frac{1}{2} \beta_{\lambda} \phi_{0}^{2} .
$$

Therefore, in order that the potential exhibits a second minimum, the function $\beta_{\lambda}$ must have passed a zero because we know that $\beta_{\lambda}$ is negative at EW scales. For the parameter set [Jeg] a zero is found at about $\mu_{\lambda} \simeq 10^{17} \mathrm{GeV}$. What happens for the two parameter sets [Jeg] and [Deg] is shown in Fig. 11, which also illustrates the significance of the radiative corrections. In the stability case the effective potential does not alter the main picture, while in the metastable case a second minimum is also missing and the potential turns unbounded from below way below the Planck regime. Since the tunneling rate to the Planck regime is exceedingly low, the EW vacuum still looks to be stable. As follows from the SM RG, because $\beta_{\lambda}$ contains parts which are not proportional to $\lambda$, the Higgs self-coupling $\lambda$ is the only SM dimensionless coupling that can change the sign with increasing energy scale.

In our case, where $\lambda(\mu)>0$ up to $M_{\mathrm{Pl}}$, in the early phase of the expanding universe, the effective potential is approximated by

$$
V(\phi) \approx V(0)+\frac{m_{\mathrm{eff}}^{2}}{2} \phi^{2}+\frac{\lambda_{\mathrm{eff}}}{24} \phi^{4},
$$

and the correction turns out not to be significant for what concerns the scenario as such. Actually, the upshot of the two-loop analysis in [129] has been that "the requirement that the electroweak vacuum remains stable turns out to be essentially identical to the requirement that $\lambda$ remains positive".

One has to keep in mind that a metastable EW ground-state in a globally unstable potential, as found in commonly accepted analyses [37, 58,70], very likely does not model what truly happens at the Planck scale. It could signal the need for an extension of the SM including new physics or that the analysis underestimates uncertainties. 

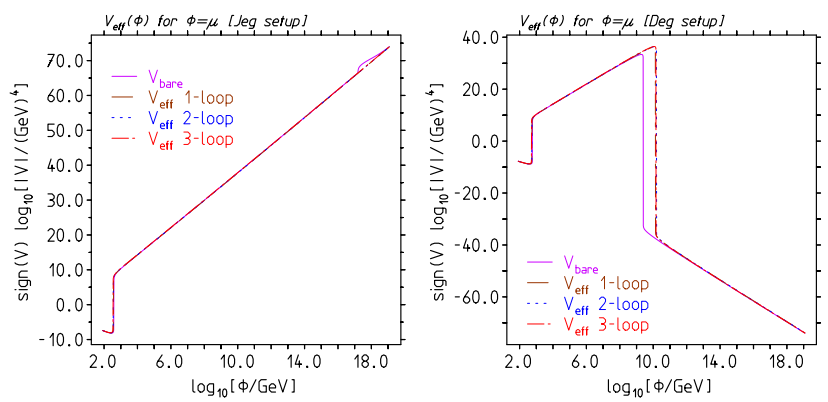

Figure 11: The effective potential including 1-,2-, and leading 3-loop [58, 131] corrections, with $\mu=\phi$ as a scale. Left: for parameter set [Jeg] (stable vacuum). Right: for parameter set [Deg] (metastable case); the EW vacuum is tunneling into the bottomless potential. The tunneling time by far exceeds the age of the universe and hence looks very stable for us.

\section{The cosmological constant - dark energy provided by the Higgs scalar}

It is crucial that in the early universe both terms in the Higgs potential are positive in order to condition slow-roll inflation during long enough time. In fact the quadratically and quartically cutoff enhanced terms in the Higgs potential enforce the condition $\frac{1}{2} \dot{\phi}^{2} \ll V(\phi)$ and given the Higgs boson pressure $p_{\phi}=\frac{1}{2} \dot{\phi}^{2}-V(\phi)$ and the Higgs energy density $\rho_{\phi}=\frac{1}{2} \dot{\phi}^{2}+V(\phi)$, we arrive at the equation of state $w=p / \rho \approx-1$ characteristic for dark energy and the equivalent CC (see e.g. [20,134-136] and references therein). A first remarkably precise measurement of the dark energy equation of state $w=-1.01 \pm 0.04$ has been obtained by the Planck mission $[16,17]$ recently (for an actual review see [137]). A more detailed study [2] shows that the enhanced Higgs boson effective mass alone actually does not provide a sufficient amount of inflation, which is required to inflate the causal CMB cone to include the full CMB sky ${ }^{23}$.

One important quantity we have not taken into account so far is the vacuum energy $V(0)=\langle V(\phi)\rangle$. A key point is that in the LEESM scenario the vacuum energy is a calculable quantity. In the symmetric phase $S U(2)$ symmetry implies that while $\langle\Phi(x)\rangle \equiv 0$ the composite field $\Phi^{+} \Phi(x)$ is a singlet such that the invariant vacuum energy is given just by simple Higgs field loops

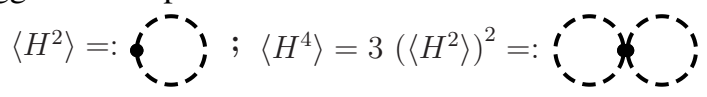

where

$$
\left\langle 0\left|\Phi^{+} \Phi\right| 0\right\rangle=\frac{1}{2}\left\langle 0\left|H^{2}\right| 0\right\rangle \equiv \frac{1}{2} \Xi ; \Xi=\frac{\Lambda_{\mathrm{Pl}}^{2}}{16 \pi^{2}} .
$$

\footnotetext{
${ }^{23}$ This is the Horizon problem: the finite age $t$ of the universe together with the finite speed of light $c$ allows us to see to distances $D_{\text {hor }}=c t$ at most. The CMB sky is much larger $\left[d_{t_{\mathrm{CMB}}} \simeq 4 \cdot 10^{7} \ell \mathrm{ly}\right]$ than the causally connected patch $\left[D_{\mathrm{CMB}} \simeq 4 \cdot 10^{5} \ell \mathrm{y}\right]$ at the time of last scattering $t_{\mathrm{CMB}} \simeq 380000 \mathrm{yrs}$ when the $\mathrm{CMB}$ decoupled from matter. As we know, no $D_{\mathrm{CMB}}$ spot shadow is distinguishable at the full CMB sky.
} 
This provides a $\mathrm{CC}$ given by

$$
V(0)=\langle V(\phi)\rangle=\frac{m^{2}}{2} \Xi+\frac{\lambda}{8} \Xi^{2} .
$$

A Wick ordering type of rearrangement of the Lagrangian also leads to a shift oft the effective mass

$$
m^{\prime 2}=m^{2}+\frac{\lambda}{2} \Xi
$$

For our values of the $\overline{\mathrm{MS}}$ input parameters the zero in the Higgs mass counter term and hence the phase transition point gets shifted downwards as follows

$$
\mu_{0} \approx 1.4 \times 10^{16} \mathrm{GeV} \rightarrow \mu_{0}^{\prime} \approx 7.7 \times 10^{14} \mathrm{GeV} .
$$

The shift is shown in the right panel of Fig. 8. We notice that the SM predicts a huge $\mathrm{CC}$ at $M_{\mathrm{Pl}}$ :

$$
\rho_{\phi} \simeq V(\phi) \sim 2.77 M_{\mathrm{Pl}}^{4} \sim 6.13 \times 10^{76} \mathrm{GeV}^{4}
$$

exhibiting a very weak scale dependence (running couplings) and we are confronted with the question how to get rid of this huge quasi-constant? Remember that $\rho_{\phi}$ has no direct dependence on $a(t)$. An intriguing structure again solves the puzzle. The effective CC counterterm has a zero, which again is a point where renormalized and bare quantities are in agreement:

$$
\rho_{\Lambda 0}=\rho_{\Lambda}+\delta \rho_{\Lambda} ; \delta \rho_{\Lambda}=\frac{\Lambda_{\mathrm{Pl}}^{4}}{\left(16 \pi^{2}\right)^{2}} X(\mu)
$$

with $X(\mu) \simeq \frac{1}{8}(2 C(\mu)+\lambda(\mu))$ which has a zero close to the zero of $C(\mu)$ when $2 C(\mu)=$ $-\lambda(\mu)$. Note that $C(\mu)=-\lambda(\mu)$ is the shifted Higgs transition point.

Again we find a matching point $\rho_{\Lambda 0}=\rho_{\Lambda}$ between the low-energy and the highenergy world. At this point, the memory of the quartic Planck scale enhancement gets lost, as it should be since we know that the low energy phase does not provide access to cutoff-effects.

Crucial point is that

$$
X(\mu)=2 C+\lambda=5 \lambda+3 g_{1}^{2}+9 g_{2}^{2}-24 y_{t}^{2}
$$

acquires positive bosonic contribution and negative fermionic ones, with different scaledependence ${ }^{24} . X$ can change a lot (pass a zero), while individual couplings are weakly scale-dependent with $y_{t}\left(M_{Z}\right) / y_{t}\left(M_{\mathrm{Pl}}\right) \sim 2.7$ the biggest and $g_{1}\left(M_{Z}\right) / g_{1}\left(M_{\mathrm{Pl}}\right) \sim 0.76$ the smallest change. Obviously, the energy dependence of any of the individual couplings would by far not be able to sufficiently diminish the originally huge cosmological constant. Only the existence of a zero in the coefficient function $X(\mu)$ is able to provide the dramatic reduction of the effective $\mathrm{CC}$, by nullifying the huge cutoff-sensitive prefactor.

At the Higgs transition point, as soon as $m^{\prime 2}<0$ for $\mu<\mu_{0}^{\prime}$, the vacuum rearrangement of the Higgs potential takes place. As a result at the minimum $\phi_{v}$ of the potential, 


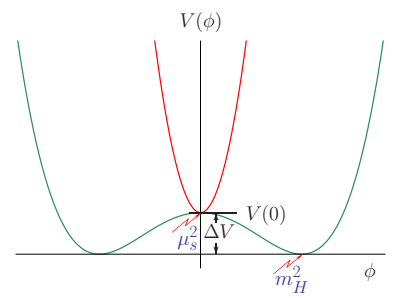

Figure 12: Vacuum rearrangement by the Higgs phase transitions. The large offset $V(0)$ essentially gets nullified at the transition point.

we should get $V(0)+V\left(\phi_{v}\right) \sim(0.00171 \mathrm{eV})^{4}$ about the observed value of today's CC (see Fig. 12). How can this be? Indeed, at the zero of $X(\mu)$ we have $\rho_{\Lambda 0}=\rho_{\Lambda}$ and one may expect that like the Higgs boson mass another free SM parameter is to be fixed by experiment here ${ }^{25}$. One might expect $\rho_{\Lambda}$ to be naturally small, since the $\Lambda_{\mathrm{Pl}}^{4}$ term is nullified at the matching point. Note that the huge cutoff prefactors act as amplifiers of small changes in the effective SM couplings. But how small we should expect the low energy effective CC to be? In fact, in the LEESM scenario, neither the Higgs boson mass nor the $\mathrm{CC}$ is really a free parameters in the low-energy world. Given the other relevant SM parameter, the Higgs self-coupling has to be constrained to a window where the Higgs potential remains stable up to the Planck scale. Similarly, the originally large $\mathrm{CC}$, which is required to provide a sufficient amount of inflation, has to get tuned down such that inflation ends up at the critical density of a flat universe. The late $\mathrm{CC}$ as part of the critical density then only can be a fraction of the latter.

\subsection{A self-organized cosmological constant?}

Implications of inflation we already outlined in Sect. 1 after Eq. (5). Our analysis of the LEESM showed that the CC is very much time dependent especially through the running of the SM parameters and phase transitions taking place in the evolution of the universe (see also the Quintessence scenario advocated in [39]). The typical problem is that in general one gets a CC that is way too big, and this looks to create a tremendous fine-tuning problem. For the SM this concerns the contribution to the vacuum density via the Higgs-field VEV in the broken phase, as well as the contributions from spontaneous breakdown of chiral symmetry, which both are much too big and even of wrong sign. Interestingly, our Higgs inflation scenario predicts a large positive DE, which actually implies that $\rho_{\text {tot }} \gg \rho_{\text {crit }}$ before inflation sets in. This means that at Planck time $k=+1$ and $\Omega_{k}$ in Eq. (7) evaluated at Planck time is large negative if $a\left(t_{\mathrm{Pl}}\right)$ is of Planck size. It is important to keep in mind that in Big Bang cosmology $\rho_{\text {tot }}$ at the beginning is always dominated by the radiation density since $\rho_{\text {rad }} \propto a(t)^{-4}$ grows fastest when $a(t)$

\footnotetext{
${ }^{24}$ Unbroken SUSY would require a perfect cancellation to happen at all scales. Broken SUSY would largely diminish the quadratic and quartic enhancements which are key effects in our scenery.

${ }^{25}$ The appearance of an non-vanishing $v$ provides a large negative contribution, which however by far does not compensate the large positive offset $\langle V(\phi)\rangle$ we have from the symmetric phase. A more accurate analysis would have to take into account subleading effects from the chiral phase transition of QCD as-well.
} 


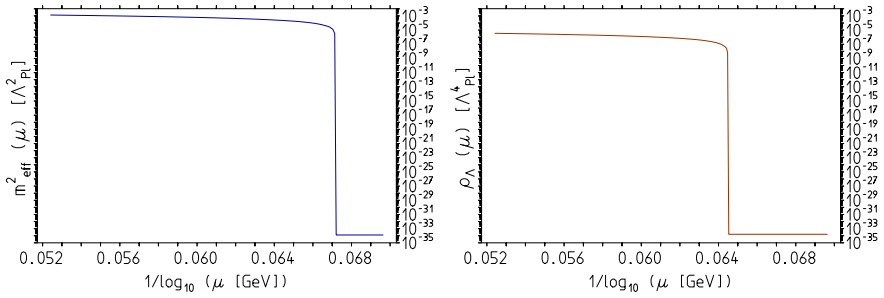

Figure 13: The Higgs potential effective $m_{\text {eff }}^{2}$ [left] and the dark energy density $\rho_{\Lambda}$ [right], in units of $\Lambda_{\mathrm{Pl}}$, as functions of "time", represented here by $1 / \log _{10} \mu$, where $\mu$ represents the energy at that time. Below the matching point $\mu_{\mathrm{CC}}$, where $\rho_{\Lambda} \simeq 1.6 \times$ $10^{-47}$ in Planck mass units, we show a scaled up $\rho_{\Lambda} \times 10^{13}$ value of the present dark energy density $\mu_{0 \Lambda}^{4}$ with $\mu_{0 \Lambda} \simeq 0.00171 \mathrm{eV}$. Note: $\rho_{\Lambda}(t)$ includes besides the large positive $V(0)$ also negative contributions from vacuum condensates, like $\Delta \rho_{\mathrm{EW}}$ from the Higgs mechanism and $\Delta \rho_{\mathrm{QCD}}$ from the chiral phase transition

gets smaller as we are going back in time. Because they also scale with inverse powers in $a(t)$, also the matter-density and the curvature terms first overshoot the CC supplied by Higgs boson system. This is possible because $\rho_{\Lambda} \sim\left(1.29 M_{\mathrm{Pl}}\right)^{4}$ is of comparatively moderate size, although extremely big relative to the critical density. However, if inflation is at work, the final vacuum density is fixed, whatever the initial density has been. Given that $\Omega_{\mathrm{tot}}=\Omega_{\Lambda}+\Omega_{\mathrm{DM}}+\Omega_{\mathrm{BM}}+\Omega_{\mathrm{rad}}=1$ with $1>\Omega_{\mathrm{DM}}>\Omega_{\mathrm{BM}}>\Omega_{\mathrm{rad}}>0$ we know that $\Omega_{\Lambda}$ being positive must be of order $\Omega_{\text {tot }}$, actually a fraction of it. As a non-vanishing $\rho_{\Lambda 0}$ at Planck time is needed, it is not unlikely that the other components contributing to the total energy density do not saturate the bound. Actually, we know that normal matter including the tiny radiation density represents about $5 \%$ of the critical density only. This means that the fine-tuning is dynamically enforced by inflation and the value of today's dark energy density

$$
\rho_{0 \Lambda}=\mu_{0 \Lambda}^{4} ; \mu_{0 \Lambda}=0.00171 \mathrm{eV}
$$

looks all but exotic. While $\Omega_{\text {rad }}$ and very likely $\Omega_{\mathrm{BM}}$ are essentially LEESM predictions if we include the $B+L$ violating dimension 6 four-fermion operators, $\Omega_{\mathrm{DM}}$ is the only missing piece which remains an open problem and definitely requires additional beyond the SM physics. This also concerns contributions from quark- and possible gluon-condensates, which we do not explicitly consider here.

Provided SM parameters indeed support a stable Higgs potential up to $M_{\mathrm{Pl}}$, inflation and the CC itself are SM ingredients leading to a highly self-consistent conspiracy which shapes the universe. Fig. 13 shows the development of the quadratically and the quartically enhanced terms in the symmetric phase of the SM, and its matching to the low energy world. 


\section{$7 \quad$ Inflation and reheating}

In contrast to standard scenarios of modeling the evolution of the early universe, SM cosmology is characterized by the fact that almost everything is known, within uncertainties of the parameters and perturbative approximations. In LEESM cosmology the form of the potential is given by the bare Higgs potential $V(\phi)=\frac{m^{2}}{2} \phi^{2}+\frac{\lambda}{24} \phi^{4}$ as part of the SM Lagrangian, the parameters are known, calculable in terms of the low energy parameters, the only unknown is the magnitude of the Higgs field. The latter must be large - trans-Planckian - in order to get the required number of $e$-folds $N$ given by

$$
N \equiv \ln \frac{a\left(t_{\mathrm{e}}\right)}{a\left(t_{\mathrm{i}}\right)}=\int_{t_{i}}^{t_{e}} H(t) \mathrm{d} t \simeq-\frac{8 \pi}{M_{\mathrm{Pl}}^{2}} \int_{\phi_{i}}^{\phi_{e}} \frac{V}{V^{\prime}} \mathrm{d} \phi .
$$

The second form is obtained using the field equation (4). Note that $N$ is determined entirely by the scalar potential. Needed is $N \approx 60$ in order to cover the CMB causal cone. By definition, $\exp N$ is the expansion factor $a\left(t_{e}\right) / a\left(t_{i}\right)=\exp H\left(t_{e}-t_{i}\right)$, where $a(t)$ is the Friedmann-Robertson-Walker radius of the universe at cosmic time $t, t_{i}$ denotes the begin of inflation and $t_{e}$ the end of inflation and $H$ the Hubble constant. For our set of $\overline{\mathrm{MS}}$ input parameters we require $\phi_{0}=\phi\left(\mu=M_{\mathrm{Pl}}\right) \approx 4.5 M_{\mathrm{Pl}}$. Shortly after start the slow-roll condition $V(\phi) \gg \frac{1}{2} \dot{\phi}^{2}$ is well satisfied, by the fact that in the symmetric phase the mass term as well as $V(0)=\langle V(\phi)\rangle$ are huge and start to dominate quickly. Because of the large initial field strength $\phi_{0}$, however, the interaction term is actually dominating for a short time after the initial Planck time $t_{\mathrm{Pl}}$. The field equation $\ddot{\phi}+3 H \dot{\phi}=-V^{\prime}(\phi)$ then predicts a dramatic decay of the field, $\phi(t)=\phi_{0} \mathrm{e}^{E_{0}\left(t-t_{0}\right)}$ with $E_{0}=\sqrt{2 \lambda} /(3 \sqrt{3} \ell) \approx 4.3 \times 10^{17} \mathrm{GeV}, V_{\text {int }} \gg V_{\text {mass }}$ and shortly after $E_{0}=m^{2} /(3 \ell \sqrt{V(0)}) \approx 6.6 \times 10^{17} \mathrm{GeV}, V_{\text {mass }} \gg V_{\text {int }}\left[\ell^{2}=8 \pi G_{N} / 3\right]$, such that in almost no time, still under slow-roll conditions, the mass term dominates and for what follows the field equation predicts an exponential decay followed by harmonic oscillation setting in. The universe thus undergoes an epoch of Gaussian inflation as confirmed by observation [21]. The time evolution is displayed in Fig. 14 and it is very interesting to see which term dominates during which time slice. Obviously, the fast decay of the Higgs field stops inflation (see Fig. 15), in spite of the fact that a CC V(0) persists to be substantial at first.

A highly non-trivial challenge is the calculation of the spectral indices

$$
\varepsilon \equiv \frac{M_{\mathrm{Pl}}^{2}}{8 \pi} \frac{1}{2}\left(\frac{V^{\prime}}{V}\right)^{2} ; \eta \equiv \frac{M_{\mathrm{Pl}}^{2}}{8 \pi} \frac{V^{\prime \prime}}{V},
$$

which sensitively depend on the form of the potential and which have been extracted from the observed $\mathrm{CMB}$ radiation fluctuation spectrum. From the theory point of view the indices are constrained by the slow-roll criteria $\varepsilon \ll 1$, ensuring $p \simeq-\rho$, and $\varepsilon, \eta \ll 1$, which ensures that the slow-roll condition hold for long enough time, while maintaining $\ddot{\phi} \ll 3 H \dot{\phi}$ before oscillations start. These conditions also should be satisfied in order to ensure the required amount of inflation. In our case, we are confronted with a SM prediction modulo the unknown Higgs field $\phi_{0}=\phi\left(\mu=M_{\mathrm{Pl}}\right)$. The calculation presented in [2] shows that a prediction of $\eta$ is delicate, but present uncertainties in predicting the bare Higgs potential at post-inflation times certainly do not allow us the 

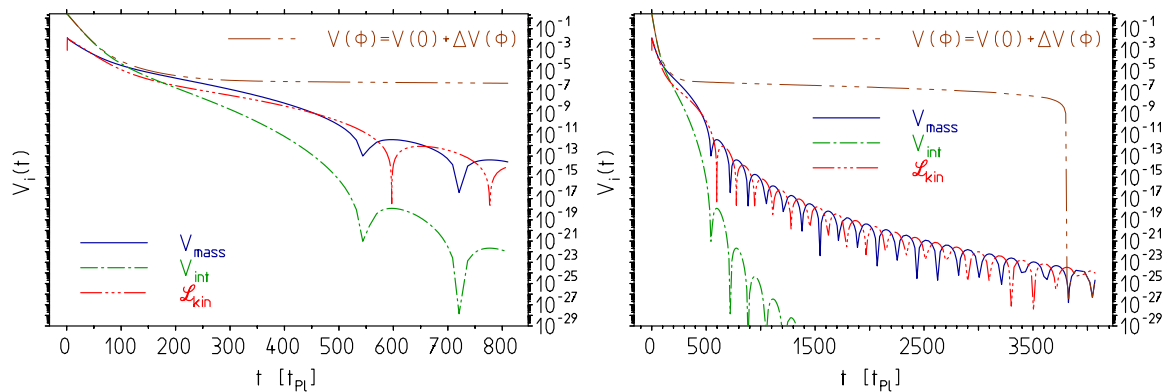

Figure 14: The evolution of the universe before the Higgs phase transition. Left: during inflation times - the mass-, interaction- and kinetic-term of the bare Lagrangian in units of $M_{\mathrm{Pl}}^{4}$ as a function of time. Right: evolution until symmetry breakdown and vanishing of the CC. After inflation the scene is characterized by a free damped harmonic oscillator behavior

draw definite conclusions. Given that there are many predictions that look to work surprisingly well, I would be surprised if the Higgs boson inflation would not work in predicting also the spectral exponents in agreement with observation at the end.

\subsection{Reheating by Higgs boson decays}

In the symmetric phase, all four Higgs fields are physical and very heavy and rather unstable. The Yukawa couplings at inflation times are pretty well known and the Higgs bosons decay predominantly (largest Yukawa couplings) into as yet massless top-antitop pairs and lighter fermion-antifermion pairs $H, \phi^{0} \rightarrow t \bar{t}, b \bar{b}, \cdots, H^{+} \rightarrow t \bar{b} \cdots, H^{-} \rightarrow$ $\bar{t} b \cdots$ and are thereby reheating the young universe, which just had been cooled down dramatically by inflation. Preheating is suppressed in SM inflation as in the symmetric phase bosonic decay channels like $H \rightarrow W W$ and $H \rightarrow Z Z$ are absent at tree level. The CP-violating decays $H^{+} \rightarrow t \bar{d}$ [rate $\left.\propto y_{t} y_{d} V_{t d}\right] H^{-} \rightarrow b \bar{u}$ [rate $\propto y_{b} y_{u} V_{u b}$ ] likely are important for baryogenesis. Closely following the Higgs transition, where $m^{2}$ in the Higgs potential changes sign, the electroweak phase transition takes place. After it, the now heavy top-quarks decay into normal matter as driven by CKM [138] couplings and phase space. At these scales the $B+L$ violating dimension 6 operators [139-141] can still play a key role for baryogenesis and together with decays like $t \rightarrow d e^{+} v$ provide $\mathrm{CP}$ violating reactions during a phase out of thermal equilibrium ${ }^{26}$. For details see $[1,2,107]$.

A very different model of Higgs inflation, which has barely something in common with our LEESM scenario, is the Minkowski-Zee-Shaposhnikov et al. [31-37] so-called non-minimal SM inflation scenario. It is based on the following points: i)

\footnotetext{
${ }^{26}$ We note that, in contrast to claims that the SM cannot explain baryogenesis, the latter looks to be well possible within the LEESM scenario, provided the EW phase-transition happens not too far below the Planck scale, at a scale $\mu_{0}$ where the mentioned dimension 6 four-fermion operators can be sufficiently effective i.e. $\left(\mu_{0} / \Lambda_{\mathrm{Pl}}\right)^{2} \sim 1.4 \times 10^{-6}$ is not too small. The observed baryon asymmetry is $\eta_{B} \sim 10^{-10}$. Remains the question of whether CP violation as given by the SM is big enough and sufficiently efficient in the new context.
} 
Einstein gravity has to be extended by adding $G_{\mu \nu} \rightarrow G_{\mu \nu}+\xi\left(\Phi^{+} \Phi\right) R g_{\mu \nu}$ to Einstein's equation. On the source side the model is assuming the renormalized low energy $T_{\mu \nu}$ supplied by the renormalized SM (no relevant operator enhancement). The new term is a direct coupling of the gauge invariant Higgs field singlet operator $\Phi^{+} \Phi$ to the scalar Ricci curvature $R$. This extra term violates the equivalence principle, yet so far without observable consequences. ii) Choose $\xi$ large enough in order to get a sufficient amount of inflation, which requires a rather large value $\xi \sim 10^{4}$. The entire inflation pattern then essentially depends on $\xi$ only (inflation "by hand"). In case $\xi=O(1)$ the added non-minimal coupling term is tiny and does not affect our LEESM or standard inflation scenarios. iii) Assume quadratic and quartic SM divergences are absent (argued by their absence in dimensional regularization (DR) and $\overline{\mathrm{MS}}$ renormalization, which is a misleading purely formal argument in my opinion). iv) Assume the SM to be in the broken phase at the Planck scale, which looks unnatural since SSB is a low energy phenomenon, which assumes the symmetry to be restored at the short distance scale!

Note: 1) It is well possible maybe even likely that such non-minimal gravity couplings of the Higgs field exist and could play a role when curvature is very high. However, the coupling $\xi$ would rather be $O(1)$ than fine-tuned to be about $\xi \sim 10^{4}$. 2) Dimensional regularization and $\overline{\mathrm{MS}}$ renormalization both are possible in perturbation theory only. There is no corresponding non-perturbative formulation (simulation on a lattice) or measuring prescription (experimental procedure). The $\overline{\mathrm{MS}}$ scheme is based on a finite part prescription (singularities nullified by hand), which can only be used to calculate quantities that do not exhibit any singularities at the end. As elaborated earlier in Sect. 4, the hierarchy problem cannot be addressed within the dimensionally regularized SM or adopting the $\overline{\mathrm{MS}}$ scheme in a renormalized environment. In other words, dimensional renormalization by no means explains the absence of power enhanced terms in a LEET scenario. These terms are there and have to be accounted for.

\section{Remark on trans-Planckian Higgs fields}

If the SM Higgs boson is the inflaton, sufficient inflation requires trans-Planckian magnitude Higgs fields at the Planck scale. At the cutoff scale, the low-energy expansion obviously gets obsolete and likely we cannot seriously argue with field monomials and the operator hierarchy appearing in the low energy expansion. What is important is that the field is decaying very fast (see Fig. 15). Formally, given a truncated series of operators in the potential, the highest power is dominating when approaching the transPlanckian regime. One then expects that for some time the $\phi^{4}$ term of the potential is dominating, the decay of the field is then exponential, for higher dimensional operators it is faster than exponential, such that the field very rapidly reaches the Planck- and sub-Planck regime. This means that the mass term is dominating after a very short period and before the kinetic term becomes relevant and slow-roll inflation ends. So fears that higher order operators in low energy effective scenarios with trans-Planckian fields would mess up things are unfounded ${ }^{27}$. Obviously, without the precise knowledge of

\footnotetext{
${ }^{27}$ As mentioned earlier, the constructive understanding of LEETs is Wilson's renormalization semi-group, based on integrating out short distance fluctuations. This produces all kinds, mostly of irrelevant higher
} 

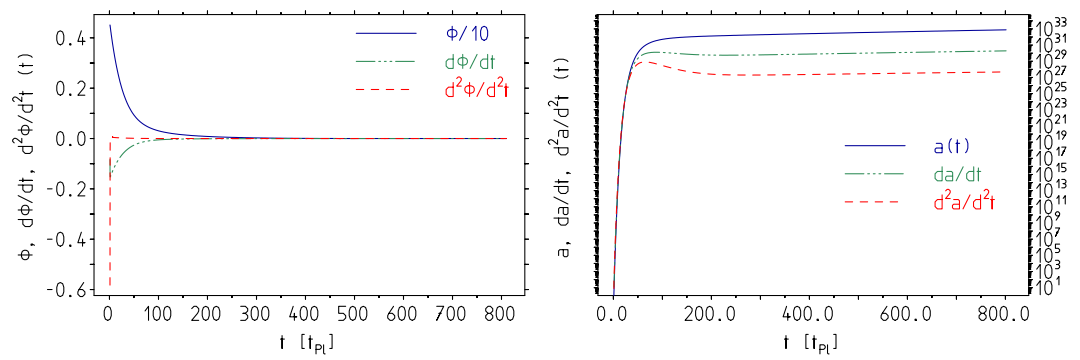

Figure 15: The trans-Planckian Higgs field at $t_{\mathrm{PI}}$ decays very fast and inflation gets stopped soon. Left: the decaying Higgs field. Right: the inflating $a(t)$.

the Planck physics, very close to the Planck scale we never will be able to make a precise prediction of what is happening. This, however, seems not to be a serious obstacle to quantitatively describe inflation and its properties as far as they can be accessed by observation. The LEESM scenario in principle predicts not only the form of the effective potential not far below the Planck scale but also its parameters and the only quantity not fixed by low energy physics is the magnitude of the field at the Planck scale. We also have shown that taking into account the running of the parameters is mandatory for understanding inflation and reheating and all that.

Trans-Planckian fields are not unnatural in a low energy effective scenario as the Planck medium exhibits a high temperature and temperature fluctuations imply amply of higher excitations forming a chaotic state. While the Planck medium will never be accessible to direct experimental tests, a phenomenological approach to constraining its effective properties is obviously possible, especially by CMB data [142].

In the extremely hot Planckian medium, the Hubble constant in the radiation dominated state with effective number $g_{*}(T)=g_{B}(T)+\frac{7}{8} g_{f}(T)=102.75$ of relativistic degrees of freedom is given by $H=\ell \sqrt{\rho} \simeq 1.66\left(k_{B} T\right)^{2} \sqrt{102.75} M_{\mathrm{Pl}}^{-1}$, at Planck time $H_{i} \simeq 16.83 M_{\mathrm{Pl}}$ such that a Higgs field of size $\phi_{i} \simeq 4.51 M_{\mathrm{Pl}}$, is not unexpectedly large and could as well also be larger.

Often it is argued that trans-Planckian fields are unnatural in particular in a LEET scenario [143]. I cannot see any argument against strong fields and LEET arguments (ordering operators with respect to a polynomial expansion and their dimension) completely lose their sense when $E / \Lambda_{\mathrm{Pl}} \gtrsim 1$.

As mentioned already, provided the Higgs field decays fast enough, towards the end of inflation, we expect the mass term to be dominant such that a Gaussian fluctuation spectrum prevails. The quasi-constant $\mathrm{CC} V(0)$ at these times mainly enters the Hubble constant $H$ and does not affect the fluctuation spectrum. As the originally large $V(0)$ get nullified at $\mu_{\mathrm{CC}}$ also the Hubble constant suffers a jump down to a value as known

order interactions. A typical example is an Ising model, which by itself seen as the basic microscopic system has simple nearest neighbor interactions only and by the low-energy expansion develops a tower of higher order operators, which at the short distance scale are simply absent altogether. Such operators don't do any harm at the intrinsic short distance scale. As a minimal fairly realistic Planck system representative in the universality class of the SM, we may consider the lattice SM, a SM generalization of lattice QCD, which also is expected to behave decently at the short distance scale. 
from standard $\Lambda \mathrm{CDM}$ cosmology.

\section{Remarks for the skeptic}

How do our results depend on the true UV completion? In other words, how realistic are the numbers I have presented?

In order to answer these questions, we have to stress once more the extreme size of the cutoff, $M_{\mathrm{Pl}}>>>>$... from what we can see! This let us look what we can explore to be ruled by fundamental principles like the Wightman axioms (the "Ten Commandments" of QFT) or extensions of them as they are imposed in deriving the renormalizable SM. In the LEESM approach, many things are much more clear-cut than in condensed matter systems, where cutoffs are directly accessible to experiments. In other words, in condensed matter systems the gap between the microscopic structure and its macroscopic manifestation is by far nowhere nearly as big as in our LEESM case. Also lattice QCD simulations differ a lot, as cutoffs are always close-by, such that lattice artifacts affect results throughout before the extrapolation to the continuum has been performed.

We also have to stress that taking actual numbers too serious is premature as long as even the realization of vacuum stability is in question. Detailed results evidently depend sensitively on accurate input values and on the perturbative approximations used for the renormalization group coefficients as well as for the matching relations needed to get the $\overline{\mathrm{MS}}$ input parameters in terms of the physical (on-shell) ones. After all, we are attempting to extrapolate over 16 orders of magnitude in the energy scale. Such an attempt may look to be megalomaniacal, but it is a bottom-up approach that appears to lead to a reasonable and very possible scenario that is able to explain and predict inflation and reheating. And it is a very modest step in relation to attempts to construct a TOE for example.

The next question is how close to $M_{\mathrm{Pl}}$ can we trust our extrapolation? It is very important to note that above the EW scale $[v \sim 250 \mathrm{GeV}]$ perturbation theory seems to works the better the closer we are near the Planck-cutoff, vacuum stability presupposed. As long as we are talking about the perturbative regime we can expand perturbative results in powers of $E / \Lambda_{\mathrm{Pl}}$ up to logarithms. Then we have full control over the cutoff-dependence to order $O\left(\left(E / \Lambda_{\mathrm{Pl}}\right)^{2}\right)$, corresponding to dim $\geq 6$ operator corrections. Effects $O\left(\left(E / \Lambda_{\mathrm{Pl}}\right)\right)$, related to dim 5 operators, only show up in special circumstances e.g. in scenarios related to generating neutrino masses and mixings and the see-saw mechanism.

The true problem comes about when we approach the Planck scale, where the expansion in $E / \Lambda_{\mathrm{Pl}}$ completely breaks down. Especially, it does not make sense to talk about a tower of operators of increasing dimensions. This does not mean that everything gets out of control. If the "ether" would be something that can be modeled by a lattice SM, implemented similar to lattice QCD, one could still make useful predictions, which eventually could be tested in cosmological phenomena. In condensed matter physics it is well known that an effective Heisenberg Hamiltonian allows one to catch essential properties of the system, although the real structure cannot be expected to be reproduced in the details. One also should keep in mind that models like 
the mentioned Ginzburg-Landau effective theory of superconductivity perfectly models the phase transition between type I, type II superconductivity and normal state, without reference to the true microscopic structure. In any case, it is always possible to find out to what extent the description fits reality.

It is well known that long-range physics manifests itself as field theory naturally from underlying classical statistical systems exhibiting short-range exchange interactions (e.g. nearest-neighbor interactions on a lattice system) [90] (for lectures on the topic see e.g. [91]). However, the Planck system not only shows typical short-range interactions. We know that it also features a long-range gravitational potential, which develops multipole excitations showing up as spin 1, spin 2 and higher modes at long distances [100].

In our context what is important is that the quadratic and quartic enhancements are persisting, as well as the running (screening or anti-screening effects) of couplings and their competition and conspiracy. Together these elements manifest themselves in the existence of the zeros of the enhanced terms, provided these zeros are not to close to the cutoff. A look at Fig. 7 shows that such effects can be dramatic fairly well below the cutoff. Again, the perturbativeness, together with the fact that leading corrections to these results are by $\operatorname{dim} 6$ operators, let us expect that results are reliable at the $10^{-4}$ level up to $10^{17} \mathrm{GeV}$, which is in the middle of the symmetric phase already. Once the phase transition has happened, the running is anyway weak and even when cutoff-effect are starting to play a role they cannot spoil the relevant qualitative features concerning triggering inflation, reheating and related phenomena.

Lattice SM simulations in the appropriate parameter range of vacuum stability, keeping top-quark Yukawa and Higgs self-coupling to behave asymptotically free, which requires to include simultaneously besides the Higgs system also the top-quark Yukawa sector and QCD, could help to investigate such problems quantitatively. Experience from lattice QCD simulations may not directly be illustrative since usually the cutoff is rather close and a crucial difference is also the true non-perturbative nature of low energy QCD.

In any case, not to include the effects related to the relevant operators $(\operatorname{dim}<4)$ simply must give wrong results. Even substantial uncertainties, which certainly show up closer to the cutoff in power-like behaved quantities, seem to be an acceptable shortcoming in comparison to not taking into account the cutoff-enhancements at all (as usually done).

\section{Summary and conclusions}

A cutoff regularized SM with the Planck mass as a cutoff is considered to exhibit the relevant features of the physical Planck world in the sense that it resides in the same universality class with respect to its long-range behavior. The SM we observe at low energy is then the emergent renormalizable effective theory of the Planck medium. All conditions that usually have to be imposed, as principles to ensure renormalizability, are emergent as a result of the low energy expansion. The relation between long distance physics and short distance physics can be controlled in principle via Wilson's renormalization semi-group. Taking into account renormalization effects and the "run- 
ning" of the parameters are mandatory in order to understand what has been happening in the evolution of the universe. The key outcome of the LEESM setting is that for appropriately tuned parameters the quadratic as well as the quartic "singularities" are nullified at a specific energy, where bosonic and fermionic effects cancel ${ }^{28}$. In the SM the corresponding zeros happen because the bosonic and the fermionic contributions are running differently, according to their respective renormalization groups, and at some point turn out to be of equal magnitude.

In this scenario the Higgs field/particle has two different functions in our world: 1) it has to render the effective low energy electroweak theory (massive vector-boson and fermion sector) renormalizable. In place of fermion mass terms, we have fermion Yukawa couplings to start with, while gauge boson mass terms enter via the kinetic Higgs term through the covariant derivative that has to include the gauge fields. In the broken low energy phase, the Higgs field forms a vacuum condensate, which provides masses to all massive fields including the Higgs boson itself. The key point is the many new Higgs field exchange-forces necessary to render the low energy amplitudes renormalizable. 2) in the symmetric phase there exist four very heavy Higgs bosons $\left(H, \phi^{0}, H^{ \pm}\right)$that generate a huge positive dark energy, as required to triggers inflation. After inflation has ended and we are out of equilibrium the Higgs bosons are decaying predominantly into the fermions pairs with largest Yukawa couplings (predominantly at this stage still massless top-antitop pairs), which provides the reheating of the inflated universe. The universe cooling further down then pushes the universe into the Higgs phase, where the particles acquire their masses. The predominating heavy quarks decay into the lighter ones, which later form the baryons and normal matter. This scenario is possible because of the quadratically enhanced Higgs boson mass which together with the quartically enhanced dark energy, shows up in the symmetric phase of the SM before the transition into the Higgs phase. The existence of such relevant operator effects, in my opinion, is supported by observed inflation patterns. Both, the hierarchy "problem" as well as the cosmological constant "problem" reflect important properties of the SM needed to understand the evolution of the early universe (for different opinions see [144-148]). Consolidation of our bottom-up path to physics near the Planck scale will sensibly depend on progress in high precision physics around the EW scale $v$. Especially, Higgs boson and top-quark factories (like FCC-ee or ILC) will play a key role in this context.

Concerning the presumed fine-tuning problem: the scales $M_{\mathrm{Pl}}$ and $v$ stand for different regimes and there is no reason why they should not be vastly different; one is related to gravity (Planck medium) the other to long-range order at low energies. It is the energy dependence of the SM interactions that triggers spontaneous symmetry breaking. The emergent SM symmetry apparently only three orders of magnitude below $M_{\mathrm{Pl}}$ gets broken by a non-symmetric ground state rearrangement. The critical point nevertheless is the actual value of $v$ that is non-vanishing only below a critical temperature. While in a condensed matter system one is adjusting the temperature by hand, the key problem seems to be that in particle physics we cannot adjust the temperature. But this the expanding universe is doing for us. In fact, the hot big bang universe provides

\footnotetext{
${ }^{28}$ Note that the locations of the nearby zeros of $\delta m_{H}^{2}$ Eq. (19) and $\delta \rho_{\Lambda}$ Eq. (39) are independent of the value of the cutoff $\Lambda$, but they depend very sensitively on the input parameters specified in Tab. 1 .
} 
a scan of the temperature spectrum and automatically triggers the phase transition at some point, as the calculations show. Temporary out of equilibrium phases do not disturb the gross behavior of this scan, but they will have to be investigated in any case. For more details I refer to my original articles [1,2] and to my Krakow Lectures [149].

The scenario I advocate requires a change of paradigm, to one where the SM with its structure is emergent from a Planck cutoff-medium following a minimal selforganized "strategy", i.e. conspiracies are taking place to make structures to show up at large distances (for a short history of development of the emergence paradigm see e.g. [152]) . This looks like a version of an anthropic principle at work. It lets look the SM to be more natural than many of the BSM scenarios we have heard about during the last about 45 years. Although the SM started to turn out to work well and to work better than ever expected, we know it cannot explain a number of observationally established facts. Yes, the SM misses dark matter, singlet neutrinos, axions and likely more, but all of these have room in an emergent scenario. This is in contrast to the top-bottom philosophy behind the most popular BSM physics scenarios like string theory, supersymmetry or grand unification, which assume that the short distance world is intrinsically highly symmetric and symmetries are broken spontaneously only because renormalizability is always assumed to be a basic law of nature. We know that the SM as seen at low energies is a spontaneously broken gauge theory, which gets more symmetric as we go to higher energies because mass operators in a high-energy expansion turn into irrelevant operators. This may have lead to a wrong perception concerning what we have to expect on the path to higher energies. This view overlooks the fact that a tower of possible symmetry breaking irrelevant operators of the low energy expansion turn into relevant contributions at high energies. Thus symmetries as seen at low energy usually do not persist at higher energies. The dream that an eternal highly symmetric "theory of everything" should sit at and above the Planck scale may not be very realistic. The opposite very probably is true, the world looks more complex the closer we look, and symmetries emerge from not resolving the detailed structure behind. And the final truth remains something we can get closer only but will never be reached.

Between my Higgs inflation scenario and the metastability scenery favored in [58, $61,70,71]$, the major difference is that for me understanding the relationship between the physical low energy parameters and the bare parameter, assumed to become the physical ones at short distances, is the mandatory premise. Most other analyses are working with a renormalized effective potential in the broken phase all the way up towards the Planck scale and do not consider cutoff effects to be physical. Power enhanced cutoff-effects, in my scenario, are triggering a phase transition between an early symmetric and a later broken phase. Higgs system conditioned inflation is possible only if in the early universe the SM is in the symmetric phase.

I think the LEESM scenario has a good chance to find its confirmation along with the lines described in this article. Many aspects need to be checked and possibly modified. Admittedly, there are many open questions, which should be investigated more thoroughly. One conclusion seems to be unavoidable, namely that the SM Higgs sector provides dark energy that affects early as well as late cosmology. Obviously, discovering SUSY, a GUT, extra-dimensions, a little Higgs extension, Technicolor or similar extensions of the SM would spoil the scene. Notably, also one more fermion family 
or one less would completely mess up everything. The sharp dependence of the Higgs vacuum stability on the SM input parameters and on possible SM extensions and the vastly different scenarios which can result as a consequence of minor shifts in parameter space makes the stable vacuum case a particularly interesting one and it could reveal the Higgs particle as "the master of the universe". After all, it is commonly accepted that dark energy is the "stuff" shaping the universe both at very early as well as at the late times.

\section{Appendix: How natural is the minimal SM?}

Often it is considered that it would be more natural to have a left-right symmetric world including mirror fermions. The following consideration, which goes back to Veltman [150], is instructive as it helps to understand why parity violation is quite natural and why QED conserves parity. It has a lot to do with the assumption of a minimal Higgs system. I reproduce a version, which I had presented in [151] some time ago. Actually, within the context of our LEESM scenario, we gain a much deeper insight, because the assumptions made are now emergent properties resulting from the low energy expansion.

In order to try to derive the SM let us make the following assumptions:

1) local field theory

2) interactions follow from a local gauge principle

3) renormalizability

4) masses derive from the minimal Higgs system

5) $v_{R}$ which we know must exist does not carry hypercharge.

Note that all points besides the last one are emergent structures in a LEESM as we may learn from [100-106] (see also Sect. 3). We admit that the last assumption looks somewhat ad hoc, but nevertheless we make it. From the above assumptions the following picture develops:

- For the gauge interactions, the simplest non-trivial possibility is that the fundamental massless matter fields group according to the simplest possibilities, into doublets and triplets, which are the fundamental representations of $S U(2)$ and $S U(3)$, besides possible singlets.

- Since fields are massless all fields can be chosen left-handed. Left-handed particles and left-handed antiparticles at this stage are uncorrelated.

- We must have pairing for particles that are going to be massive, since a mass term (we ignore the possibility to have Majorana fields here) has the form $\bar{\psi} \psi=$ $\bar{\psi}_{L} \psi_{R}+\bar{\psi}_{R} \psi_{L}$. Notice that for massive particles only, we know which left-handed antiparticle belongs to which left-handed particle to form a Dirac field.

- For $S U(3)_{c}$ triplets we must have pairing in order to avoid axial anomalies. $S U(3)$ is the simplest group having complex representations. This allows putting particles in 3 and antiparticles in the inequivalent $3^{*}$. As a consequence a rich color singlet structure ( $\equiv$ hadron spectrum) results. Furthermore, confinement requires $S U(3)_{c}$ to be unbroken! 
- $S U(2)_{L}$ is anomaly free and hence there is no anomaly condition associated with this group. To generate mass we have to break $S U(2)_{L}$ by a Higgs mechanism. The simplest and natural possibility is to choose one Higgs field in the fundamental representation of $S U(2)_{L}$. There is no hypercharge for the moment. The Higgs field may be written in the form

$$
\Phi_{b}=\widetilde{\Phi} \chi_{b} ; \chi_{b}=\left(\begin{array}{l}
0 \\
1
\end{array}\right)
$$

in terms of a $2 \times 2$ matrix field $\left(\tau_{i}, i=1,2,3\right.$ the Pauli matrices $)$

$$
\widetilde{\Phi}=\frac{1}{\sqrt{2}}\left(H_{s}+\mathrm{i} \tau_{i} \phi_{i}\right) .
$$

The covariant derivative being given by

$$
D_{\mu} \Phi_{b}=\left(\partial_{\mu}-\mathrm{i} \frac{g}{2} \tau_{a} W_{\mu a}\right) \Phi_{b},
$$

and the $S U(2)$ invariant renormalizable Higgs system

$$
\mathcal{L}_{\mathrm{Higgs}}=\left(D_{\mu} \Phi_{b}\right)^{+}\left(D^{\mu} \Phi_{b}\right)-\lambda\left(\Phi_{b}^{+} \Phi_{b}\right)^{2}+\mu^{2}\left(\Phi_{b}^{+} \Phi_{b}\right),
$$

exhibits an extra global $S U(2)_{R}$-symmetry $\chi_{b} \rightarrow V^{+} \chi_{b}$. One easily checks that the transformation

$$
\widetilde{\Phi} \rightarrow U(x) \widetilde{\Phi} V^{+},
$$

with $U(x) \in S U(2)_{L, \text { local }}, V \in S U(2)_{R, \text { global }}$ leaves the Higgs Lagrangian invariant. This implies that the fields $\left(W^{+}, W_{3}, W^{-}\right)$form a weak isospin triplet with $M_{Z}=M_{W^{ \pm}}$.

Now consider the fermions (still no hypercharge). Since $L_{f}$ and $\Phi_{b}$ are $S U(2)$ doublets there also must exist singlet fermions $R_{f}$, otherwise we would not be able to write down an invariant and renormalizable fermion-Higgs coupling. Therefore $S U(2)_{L}$ must be parity violating of V-A-type! The Yukawa term has the general form

$$
\mathcal{L}_{\text {Yukawa }}=-\bar{L}_{f} \widetilde{\Phi}\left(\begin{array}{ll}
g_{11} & g_{12} \\
g_{21} & g_{22}
\end{array}\right) R_{f}+\text { h.c. },
$$

with 4 complex couplings $g_{i j}$ and $R_{f}$ a "doublet" having two right-handed singlets as entries. Although we have not used hypercharge to restrict these couplings the existence of a global $S U(2)_{R}$-symmetry of the Higgs system allows us to transform the Yukawa couplings

$$
\widetilde{\Phi}(\cdot) R_{f} \rightarrow \widetilde{\Phi} V^{+}(\cdot) W R_{f}
$$

to standard form, $V^{+}(\cdot) W=$ real diagonal. Since $V \in S U(2)_{R}$ has 3 parameters and $W$ is an arbitrary unitary matrix with 4 parameters we end up with one free 
parameter such that the system exhibits a global $U(1)$ invariance. This is not surprising since in the unitary gauge we always can end up only with $\mathcal{L}_{\text {Yukawa }}$ in the simple standard form

$$
\mathcal{L}_{\text {Yukawa }}=-\sum_{f} m_{f} \bar{\psi}_{f} \psi_{f}\left(1+\frac{H}{v}\right) .
$$

- The global $U(1)$ which is a consequence of the minimal Higgs mechanism may be interpreted as a global $U(1)_{Y}$. We are free to assign $Y=1$ to $\Phi_{b}$, which means nothing else than that we measure $Y$ in units of the $\Phi_{b}$ - hypercharge. Then

$$
\Phi_{t}=\widetilde{\Phi} \chi_{t} ; \chi_{t}=\left(\begin{array}{l}
1 \\
0
\end{array}\right)
$$

has $Y=-1$, and we may write $\widetilde{\Phi}=\left(\Phi_{b}, \Phi_{t}\right)$. Since we have the global $U(1)_{Y}$ for free, we may assume this symmetry to be local. The covariant derivative for $\widetilde{\Phi}$ now reads

$$
D_{\mu} \widetilde{\Phi}=\partial_{\mu} \widetilde{\Phi}+\mathrm{i} \frac{g^{\prime}}{2} B_{\mu} \widetilde{\Phi} \tau_{3}-\mathrm{i} \frac{g}{2} \tau_{a} W_{\mu a} \widetilde{\Phi}
$$

and we find back the usual Higgs Lagrangian

$$
\begin{aligned}
\mathcal{L}_{\text {Higgs }}= & \frac{1}{2}\left(\partial_{\mu} H \partial^{\mu} H\right)+\frac{(H+v)^{2}}{2 v^{2}}\left(M_{Z}^{2} Z_{\mu} Z^{\mu}+2 M_{W}^{2} W_{\mu}^{+} W^{-\mu}\right) \\
& -\frac{\lambda}{4} H^{4}-\lambda v H^{3}-\frac{1}{2} m_{H}^{2} H^{2} .
\end{aligned}
$$

The 3 real fields $\phi_{a} a=1,2,3$ could and have been gauged away and only 3 out of 4 gauge fields can acquire a mass. Hence there must exist one massless field, the photon! Evidently we obtain the relations $g^{\prime}=g \tan \Theta_{W}$ and $\rho=$ $M_{W}^{2} /\left(M_{Z}^{2} \cos ^{2} \Theta_{W}\right)=1$ ! instead of $M_{Z}=M_{W^{ \pm}}$when $g^{\prime}=0$.

Now, what can we say about the hypercharge of the fermions?

A left-handed doublet transforms like

$$
L \rightarrow e^{\mathrm{i} \frac{g^{\prime}}{2} Y_{L}} L
$$

where $Y_{L}$ is arbitrary. By inspection of $\mathcal{L}_{\text {Yukawa }}$ we find for the hypercharges of the singlets: $\psi_{1 R}$ must have $Y_{1 R}=Y_{L}+1$ and $\psi_{2 R}$ must have $Y_{2 R}=Y_{L}-1$. One consequence is that $U(1)_{Y}$ must violate parity. The astonishing thing is that the fermion current which couples to the photon preserves parity. By inspection we find

$$
\begin{aligned}
& D_{\mu} L_{f}=\left(\partial_{\mu}-\mathrm{i} \frac{g^{\prime}}{2} Y_{L} B-\mu-\mathrm{i} \frac{g}{2} \tau_{3} W_{\mu 3}-\cdots\right) L_{f}, \\
& D_{\mu} R_{f}=\left(\partial_{\mu}-\mathrm{i} \frac{g^{\prime}}{2} Y_{L} B-\mu-\mathrm{i} \frac{g}{2} \tau_{3} B_{\mu}-\cdots\right) R_{f},
\end{aligned}
$$


and the couplings of $L_{f}$ and $R_{f}$ to $A_{\mu}$ read

$$
\begin{array}{ll}
L_{f}: & -\mathrm{i}\left(g \sin \Theta_{W} \frac{\tau_{3}}{2}+g^{\prime} \cos \Theta_{W} \frac{Y_{L}}{2}\right) A_{\mu} \\
R_{f}: & -\mathrm{i}\left(g^{\prime} \cos \Theta_{W} \frac{\tau_{3}}{2}+g^{\prime} \cos \Theta_{W} \frac{Y_{L}}{2}\right) A_{\mu} .
\end{array}
$$

Because we have $g^{\prime} \cos \Theta_{W}=g \sin \Theta_{W}=e$ we find the Gell-Mann-Nishijima relation

$$
Q=T_{3}+\frac{Y}{2}
$$

as a consequence of a minimal Higgs structure! What we find is that, whatever the hypercharge of $L_{f}$ is, $L_{f}$ and $R_{f}$ must couple identically to photons. Thus QED must be parity conserving! Furthermore, the charges of the upper (1) and lower (2) components of the doublets satisfy

$$
Q_{L i}=Q_{R i}, Q_{1}-Q_{2}=1 \text { and } Q_{1}+Q_{2}=Y_{L} .
$$

So far we have no charge quantization. Here we need the last assumption.

- If $v_{R}$ does not couple to the $U(1)$ gauge field, we have to set $Y_{v R}=0$ and consequently we must have $Y_{v L}=-1=Y_{\ell L}=0$ and $Q_{v}=0, Q_{\ell}=-1$. For the $U(1)_{Y}$ anomaly cancellation we need lepton-quark duality and the charges of the quarks must have their known values if they appear in three colors. One thus must have the usual charge quantization.

We finally summarize the consequences of the assumptions stated above:

- breaking $S U(2)_{L}$ by a minimal Higgs automatically leads to a global $U(1)_{Y}$, which can be gauged

- parity violation of $S U(2)_{L}$

- $\rho=M_{W}^{2} /\left(M_{Z}^{2} \cos ^{2} \Theta_{W}\right)=1$

- the existence of the photon

- parity conservation of QED

- the validity of the Gell-Mann-Nishijima relation

- family structure

- charge quantization

We do not know why right-handed neutrinos are sterile i.e. do not couple to gauge bosons. In the SM of electroweak interactions, neutrinos originally were assumed to 
be massless i.e. that right-handed neutrinos did not exist. This is definitely ruled out by the observation of neutrino oscillations ${ }^{29}$.

I think this reasoning is able to help understanding how various excitations in the chaotic Planck medium develop a pattern like the SM as a low energy effective structure. Renormalizability as a consequence of the low energy expansion and the very large gap between the EW and the Planck scales plus a certain minimality (not too little but not too much e.g. only up to symmetry triplets) determines the SM structure without much freedom. After all, minimality is not a new concept in physic as we know from the principle of least action. Three fermion families are required in order $\mathrm{CP}$ violation emerges in a natural way, and to make baryogenesis eventually possible within the LEESM scenario as addressed in Sect. 7. We have been emphasizing the high self-consistency of the SM where all essential structures look to be emergent properties in the low energy effective viewport of a cutoff-system residing at the Planck scale. "What is not capable of surviving at long distances does not exist there" (Darwin revisited).

Acknowledgments:

I thank the organizers of the Naturalness, Hierarchy and Fine Tuning Workshop, at the RWTH Aachen, for the kind invitation and the support.

\section{References}

[1] F. Jegerlehner, The Standard model as a low-energy effective theory: what is triggering the Higgs mechanism?, Acta Phys. Polon. B 45, 1167 (2014) [arXiv:1304.7813 [hep-ph]].

[2] F. Jegerlehner, Higgs inflation and the cosmological constant, Acta Phys. Polon. B 45, 1215 (2014) [arXiv:1402.3738 [hep-ph]].

[3] G. 't Hooft, Naturalness, chiral symmetry, and spontaneous chiral symmetry breaking, NATO Adv. Study Inst. Ser. B Phys. 59, 135 (1980).

[4] M. J. G. Veltman, The Infrared - Ultraviolet Connection, Acta Phys. Polon. B 12, 437 (1981).

[5] R. Decker, J. Pestieau, Lepton self-mass, Higgs scalar and heavy quark masses, hep-ph/0512126.

[6] M. S. Al-sarhi, I. Jack, D. R. T. Jones, Quadratic divergences in gauge theories, Z. Phys. C 55, 283 (1992).

\footnotetext{
${ }^{29}$ We note that right-handed neutrinos have been expected to exist from the point of view of the SM gauge symmetry. They have been excluded by imposing additional global flavor conservation, because the latter had not been observed within the achieved experimental accuracy. In fact flavor violation is so tiny that a direct observation still is missing. The corresponding smallness of neutrino masses very likely is a consequence of a see-saw mechanism, which could be triggered within the SM when the singlets would be of Majorana type. Majorana mass terms are not protected by any symmetry and therefore would be expected to be related to the Planck scale in a similar way as the Higgs field mass in the symmetric phase.
} 
[7] Y. Hamada, H. Kawai, K.Y. Oda, Bare Higgs mass at Planck scale, Phys. Rev. D 87, 053009 (2013).

[8] D. R. T. Jones, The quadratic divergence in the Higgs mass revisited, Phys. Rev. D 88, 098301 (2013).

[9] C. Wetterich, Fine Tuning Problem and the Renormalization Group, Phys. Lett. 140B, 215 (1984).

[10] S. L. Glashow, Partial Symmetries of Weak Interactions, Nucl. Phys. 22, 579 (1961).

[11] S. Weinberg, A Model of Leptons, Phys. Rev. Lett. 19, 1264 (1967).

[12] H. Fritzsch, M. Gell-Mann, H. Leutwyler, Advantages of the Color Octet Gluon Picture, Phys. Lett. 47, 365 (1973);

S. Weinberg, Nonabelian Gauge Theories of the Strong Interactions, Phys. Rev. Lett. 31, 494 (1973).

[13] Mather, J.C. et al., A preliminary measurement of the cosmic microwave background spectrum by the Cosmic Background Explorer (COBE) satellite, Astrophys. J. (Letter) 354, 37 (1990).

[14] Smoot, G. et al., Structure in the COBE differential microwave radiometer first year maps, Astrophys. J (Letters) 396, 1 (1992).

[15] C. L. Bennett et al. [WMAP Collaboration], Nine-Year Wilkinson Microwave Anisotropy Probe (WMAP) Observations: Final Maps and Results, Astrophys. J. Suppl. 208, 20 (2013).

[16] P. A. R. Ade et al. [Planck Collaboration], Planck 2013 results. I. Overview of products and scientific results, Astron. Astrophys. 571, A1 (2014); Planck 2013 results. XVI. Cosmological parameters, Astron. Astrophys. 571, A16 (2014).

[17] R. Adam et al. [Planck Collaboration], Planck 2015 results. I. Overview of products and scientific results, Astron. Astrophys. 594, A1 (2016).

[18] A. G. Riess et al. [Supernova Search Team], Observational evidence from supernovae for an accelerating universe and a cosmological constant, Astron. J. 116, 1009 (1998).

[19] S. Perlmutter et al. [Supernova Cosmology Project Collaboration], Measurements of Omega and Lambda from 42 high redshift supernovae, Astrophys. J. 517, 565 (1999).

[20] S. D. Bass, The Cosmological Constant Puzzle: Vacuum Energies from QCD to Dark Energy, Acta Phys. Polon. B 45, 1269 (2014).

[21] P. A. R. Ade et al. [Planck Collaboration], Planck 2013 Results. XXIV. Constraints on primordial non-Gaussianity, Astron. Astrophys. 571, A24 (2014). 
[22] J. Dreitlein, Broken symmetry and the cosmological constant, Phys. Rev. Lett. 33, 1243 (1974).

[23] J. E. Felten, R. Isaacman, Scale factors $R(t)$ and critical values of the cosmological constant Lambda in Friedmann universes, Rev. Mod. Phys. 58, 689 (1986).

[24] V. Sahni, A. A. Starobinsky, The Case for a positive cosmological Lambda term, Int. J. Mod. Phys. D 9, 373 (2000).

[25] A. H. Guth, The Inflationary Universe: A Possible Solution to the Horizon and Flatness Problems, Phys. Rev. D 23, 347 (1981).

[26] A. A. Starobinsky, A New Type of Isotropic Cosmological Models Without Singularity, Phys. Lett. B 91, 99 (1980).

[27] A. Albrecht, P. J. Steinhardt, Cosmology for Grand Unified Theories with Radiatively Induced Symmetry Breaking, Phys. Rev. Lett. 48, 1220 (1982).

[28] A. D. Linde, A New Inflationary Universe Scenario: A Possible Solution of the Horizon, Flatness, Homogeneity, Isotropy and Primordial Monopole Problems, Phys. Lett. B 108, 389 (1982); Chaotic Inflation, Phys. Lett. B 129, 177 (1983).

[29] E. W. Kolb, M. S. Turner, The Early Universe, Front. Phys. 69, 1 (1990).

[30] S. Weinberg, Cosmology, Oxford, UK: Oxford Univ. Pr. (2008) 593 p.

[31] P. Minkowski, On the Spontaneous Origin of Newton's Constant, Phys. Lett. 71B, 419 (1977).

[32] A. Zee, A Broken Symmetric Theory of Gravity, Phys. Rev. Lett. 42, 417 (1979).

[33] F. Bezrukov, M. Shaposhnikov, The Standard Model Higgs boson as the inflaton, Phys. Lett. B 659, 703 (2008).

[34] J. L. F. Barbon, J. R. Espinosa, On the Naturalness of Higgs Inflation, Phys. Rev. D 79, 081302 (2009).

[35] F. Bezrukov, A. Magnin, M. Shaposhnikov, S. Sibiryakov, Higgs inflation: consistency and generalisations, JHEP 1101, 016 (2011).

[36] F. Bezrukov, M. Shaposhnikov, Higgs inflation at the critical point, Phys. Lett. B 734, 249 (2014).

[37] F. Bezrukov, J. Rubio, M. Shaposhnikov, Living beyond the edge: Higgs inflation and vacuum metastability, Phys. Rev. D 92, 083512 (2015).

[38] Y. Hamada, H. Kawai, K. y. Oda, S. C. Park, Higgs Inflation is Still Alive after the Results from BICEP2, Phys. Rev. Lett. 112, 241301 (2014);

Y. Hamada, H. Kawai, K. Y. Oda, Eternal Higgs inflation and the cosmological constant problem, Phys. Rev. D 92, 045009 (2015). 
[39] R. Peccei, J. Solà, C. Wetterich, Adjusting the Cosmological Constant Dynamically: Cosmons and a New Force Weaker Than Gravity, Phys. Lett. B 195, 183 (1987);

C. Wetterich, Cosmologies with Variable Newton's 'Constant', Nucl. Phys. B 302, 645 (1988); Cosmology and the Fate of Dilatation Symmetry, Nucl. Phys. B 302, 668 (1988); Inflation, quintessence, and the origin of mass, Nucl. Phys. B 897, 111 (2015).

[40] G. Aad et al. [ATLAS Collab.], Observation of a new particle in the search for the Standard Model Higgs boson with the ATLAS detector at the LHC, Phys. Lett. B 716, 1 (2012); A particle consistent with the Higgs Boson observed with the ATLAS Detector at the Large Hadron Collider, Science 338, 1576 (2012).

[41] S. Chatrchyan et al. [CMS Collab.], Observation of a new boson at a mass of $125 \mathrm{GeV}$ with the CMS experiment at the LHC, Phys. Lett. B 716, 30 (2012); A new boson with a mass of $125-\mathrm{GeV}$ observed with the CMS experiment at the Large Hadron Collider, Science 338, 1569 (2012).

[42] F. Englert, R. Brout, Broken Symmetry and the Mass of Gauge Vector Mesons, Phys. Rev. Lett. 13, 321 (1964).

[43] P. W. Higgs, Broken symmetries, massless particles and gauge fields, Phys. Lett. 12, 132 (1964).

[44] N. Cabibbo, L. Maiani, G. Parisi, R. Petronzio, Bounds on the Fermions and Higgs Boson Masses in Grand Unified Theories, Nucl. Phys. B 158, 295 (1979).

[45] P. Q. Hung, Vacuum Instability and New Constraints on Fermion Masses, Phys. Rev. Lett. 42, 873 (1979).

[46] M. Lindner, Implications of Triviality for the Standard Model, Z. Phys. C 31, 295 (1986).

[47] B. Grzadkowski, M. Lindner, Stability of Triviality Mass Bounds in the Standard Model, Phys. Lett. B 178, 81 (1986).

[48] M. Lindner, M. Sher, H. W. Zaglauer, Probing Vacuum Stability Bounds at the Fermilab Collider, Phys. Lett. B 228, 139 (1989).

[49] M. Sher, Electroweak Higgs Potentials and Vacuum Stability, Phys. Rept. 179, 273 (1989).

[50] T. Hambye, K. Riesselmann, Matching conditions and Higgs mass upper bounds revisited, Phys. Rev. D 55, 7255 (1997).

[51] J. A. Casas, J. R. Espinosa, M. Quiros, Improved Higgs mass stability bound in the standard model and implications for supersymmetry, Phys. Lett. B 342, 171 (1995); Standard model stability bounds for new physics within LHC reach, Phys. Lett. B 382, 374 (1996). 
[52] J. R. Espinosa, M. Quiros, Improved metastability bounds on the standard model Higgs mass, Phys. Lett. B 353, 257 (1995).

[53] B. Schrempp, M. Wimmer, Top quark and Higgs boson masses: Interplay between infrared and ultraviolet physics, Prog. Part. Nucl. Phys. 37, 1 (1996).

[54] G. Isidori, G. Ridolfi, A. Strumia, On the metastability of the standard model vacuum, Nucl. Phys. B 609, 387 (2001).

[55] J. R. Espinosa, G. F. Giudice, A. Riotto, Cosmological implications of the Higgs mass measurement, JCAP 0805, 002 (2008) [arXiv:0710.2484].

[56] J. Ellis, J. R. Espinosa, G. F. Giudice, A. Höcker, A. Riotto, The Probable Fate of the Standard Model, Phys. Lett. B 679, 369 (2009).

[57] B. Feldstein, L. J. Hall, T. Watari, Landscape Prediction for the Higgs Boson and Top Quark Masses, Phys. Rev. D 74, 095011 (2006).

[58] G. Degrassi, S. Di Vita, J. Elias-Miro, J. R. Espinosa, G. F. Giudice, G. Isidori, A. Strumia, Higgs mass and vacuum stability in the Standard Model at NNLO, JHEP 1208, 098 (2012).

[59] M. Shaposhnikov, C. Wetterich, Asymptotic safety of gravity and the Higgs boson mass, Phys. Lett. B 683, 196 (2010).

[60] M. Holthausen, K. S. Lim, M. Lindner, Planck scale Boundary Conditions and the Higgs Mass, JHEP 1202, 037 (2012).

[61] F. Bezrukov, M. Y. Kalmykov, B. A. Kniehl, M. Shaposhnikov, Higgs Boson Mass and New Physics, JHEP 1210, 140 (2012).

[62] S. Alekhin, A. Djouadi, S. Moch, The top quark and Higgs boson masses and the stability of the electroweak vacuum, Phys. Lett. B 716, 214 (2012).

[63] L. N. Mihaila, J. Salomon, M. Steinhauser, Gauge Coupling Beta Functions in the Standard Model to Three Loops, Phys. Rev. Lett. 108, 151602 (2012).

[64] A. V. Bednyakov, A. F. Pikelner, V. N. Velizhanin, Anomalous dimensions of gauge fields and gauge coupling $\beta$-functions in the Standard Model at three loops, JHEP 1301, 017 (2013); Yukawa coupling $\beta$-functions in the Standard Model at three loops, Phys. Lett. B 722, 336 (2013); Higgs self-coupling $\beta$ function in the Standard Model at three loops, Nucl. Phys. B 875, 552 (2013); Three-loop Higgs self-coupling $\beta$-function in the Standard Model with complex Yukawa matrices, Nucl. Phys. B 879, 256 (2014); Three-loop SM $\beta$-functions for matrix Yukawa couplings, Phys. Lett. B 737, 129 (2014).

[65] K. G. Chetyrkin, M. F. Zoller, Three-loop $\beta$-functions for top-Yukawa and the Higgs self-interaction in the Standard Model, JHEP 1206, 033 (2012); $\beta$ function for the Higgs self-interaction in the Standard Model at three-loop level, JHEP 1304, 091 (2013). 
[66] J. Fleischer, F. Jegerlehner, Radiative Corrections to Higgs Decays in the Extended Weinberg-Salam Model, Phys. Rev. D 23, 2001 (1981).

[67] A. Sirlin, R. Zucchini, Dependence of the Quartic Coupling $\bar{h}_{\overline{\mathrm{MS}}}(M)$ on $m_{H}$ and the Possible Onset of New Physics in the Higgs Sector of the Standard Model, Nucl. Phys. B 266, 389 (1986).

[68] F. Jegerlehner, M. Yu. .Kalmykov, O. Veretin, $\overline{\mathrm{MS}}$ versus pole masses of gauge bosons: Electroweak bosonic two loop corrections, Nucl. Phys. B 641, 285 (2002); Full two loop electroweak corrections to the pole masses of gauge

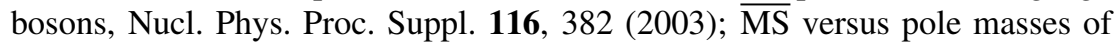
gauge bosons. 2. Two loop electroweak fermion corrections, Nucl. Phys. B 658, 49 (2003).

[69] F. Jegerlehner, M. Yu. Kalmykov, B.A. Kniehl, On the difference between the pole and the $\overline{\mathrm{MS}}$ masses of the top quark at the electroweak scale, Phys.Lett. B 722, 123 (2013); Self-consistence of the Standard Model via the renormalization group analysis, J. Phys. Conf. Ser. 608, 012074 (2015).

[70] D. Buttazzo, G. Degrassi, P. P. Giardino, G. F. Giudice, F. Sala, A. Salvio, A. Strumia, Investigating the near-criticality of the Higgs boson, JHEP 1312, 089 (2013).

[71] A. V. Bednyakov, B. A. Kniehl, A. F. Pikelner, O. L. Veretin, Stability of the Electroweak Vacuum: Gauge Independence and Advanced Precision, Phys. Rev. Lett. 115, 201802 (2015).

[72] B. A. Kniehl, A. F. Pikelner, O. L. Veretin, Two-loop electroweak threshold corrections in the Standard Model, Nucl. Phys. B 896, 19 (2015).

[73] S. P. Martin, Matching relations for decoupling in the Standard Model at two loops and beyond, Phys. Rev. D 99, 033007 (2019);

S. P. Martin, D. G. Robertson, Higgs boson mass in the Standard Model at twoloop order and beyond, Phys. Rev. D 90, 073010 (2014);

S. P. Martin, Pole Mass of the $\mathrm{W}$ Boson at Two-Loop Order in the Pure $\overline{M S}$ Scheme, Phys. Rev. D 91, 114003 (2015); Z-Boson Pole Mass at Two-Loop Order in the Pure $\overline{M S}$ Scheme, Phys. Rev. D 92, 014026 (2015).

[74] M. Awramik, M. Czakon, Complete two loop electroweak contributions to the muon lifetime in the standard model, Phys. Lett. B 568, 48 (2003).

[75] M. Awramik, M. Czakon, A. Freitas, G. Weiglein, Complete two-loop electroweak fermionic corrections to $\sin ^{2} \theta_{\text {eff }}^{\text {lept }}$ and indirect determination of the Higgs boson mass, Phys. Rev. Lett. 93, 201805 (2004);

M. Awramik, M. Czakon, A. Freitas, Bosonic corrections to the effective weak mixing angle at $O\left(\alpha^{2}\right)$, Phys. Lett. B 642, 563 (2006); Electroweak two-loop corrections to the effective weak mixing angle, JHEP 0611, 048 (2006). 
[76] S. Jadach, W. Płaczek, M. Skrzypek, B. F. L. Ward, S. A. Yost, The path to $0.01 \%$ theoretical luminosity precision for the FCC-ee, Phys. Lett. B 790, 314 (2019).

[77] E. Accomando et al. [ECFA/DESY LC Physics Working Group], Physics with $e^{+} e^{-}$linear colliders, Phys. Rept. 299, 1 (1998);

J. A. Aguilar-Saavedra et al. [ECFA/DESY LC Physics Working Group], TESLA: The Superconducting electron positron linear collider with an integrated x-ray laser laboratory. Technical design report. Part 3. Physics at an e+elinear collider, hep-ph/0106315.

[78] P. Azzi et al., Physics Behind Precision, arXiv:1703.01626 [hep-ph]; Theory report on the 11th FCC-ee workshop, 8-11 January 2019, CERN, Geneva, to appear.

[79] M. Beneke, P. Marquard, P. Nason, M. Steinhauser, On the ultimate uncertainty of the top quark pole mass, Phys. Lett. B 775, 63 (2017).

[80] T. Appelquist, J. Carazzone, Infrared Singularities and Massive Fields, Phys. Rev. D 11, 2856 (1975).

[81] M. Faisst, J. H. Kühn, O. Veretin, Pole versus MS mass definitions in the electroweak theory, Phys. Lett. B 589, 35 (2004).

[82] J. C. Taylor, Gauge Theories of Weak Interactions, Cambridge Monographs on Mathematical Physics, Cambridge University Press, 1976, 167p.

[83] E. Kraus, Renormalization of the Electroweak Standard Model to All Orders, Annals Phys. 262, 155 (1998).

[84] F. Jegerlehner, Variations on Photon Vacuum Polarization, arXiv:1711.06089 [hep-ph].

[85] F. Jegerlehner, Renormalization Scheme Dependence Of Electroweak Radiative Corrections, in Radiative corrections: Results and Perspectives. Proceedings, NATO Advanced Research Workshop, Brighton, UK, July 10-14, 1989, eds. N. Dombey and F. Boudjema, NATO Sci. Ser. B 233, 185 (1990).

[86] H. D. Politzer, Reliable Perturbative Results for Strong Interactions?, Phys. Rev. Lett. 30, 1346 (1973);

D. Gross, F. Wilczek, Ultraviolet Behavior of Nonabelian Gauge Theories, Phys. Rev. Lett. 30, 1343 (1973).

[87] J. Louis et al.: String Theory: An Overview, Lect. Notes Phys. 721, 289323 (2007).

[88] S. L. Glashow, J. Iliopoulos, L. Maiani, Weak Interactions with Lepton-Hadron Symmetry, Phys. Rev. D 2, 1285 (1970).

[89] M. Czakon, J. Gluza, F. Jegerlehner, M. Zrałek, Confronting electroweak precision measurements with new physics models, Eur. Phys. J. C 13, 275 (2000). 
[90] K. G. Wilson, Renormalization group and critical phenomena. 1. Renormalization group and the Kadanoff scaling picture, Phys. Rev. B 4, 3174 (1971); Renormalization group and critical phenomena. 2. Phase space cell analysis of critical behavior, Phys. Rev. B 4, 3184 (1971).

[91] F. Jegerlehner, An Introduction to the Theory of Critical Phenomena and the Renormalization Group, Preprint, ZIF Universität, Bielefeld, May 1976. 158pp. Lausanne Lectures http://www-com.physik.hu-berlin.de/ fjeger/ LausanneLectures 1 .pdf.

[92] F. Jegerlehner, On the Construction of Renormalized Field Theories from Cutoff and Lattice Models, Phys. Rev. D 16, 397 (1977).

[93] C. R. Ahn, M. E. Peskin, B. W. Lynn, S. B. Selipsky, Delayed Unitarity Cancellation and Heavy Particle Effects in $e^{+} e^{-} \rightarrow W^{+} W^{-}$, Nucl. Phys. B 309, 221 (1988).

[94] C. N. Yang, R. L. Mills, Conservation of Isotopic Spin and Isotopic Gauge Invariance, Phys. Rev. 96, 191 (1954).

[95] G. 't Hooft, Renormalization of Massless Yang-Mills Fields, Nucl. Phys. B 33, 173 (1971); Renormalizable Lagrangians for Massive Yang-Mills Fields, Nucl. Phys. B 35, 167 (1971);

G. 't Hooft, M. Veltman, Combinatorics of gauge fields, Nucl. Phys. B 50, 318 (1972).

[96] M. Lüscher, P. Weisz, Is There a Strong Interaction Sector in the Standard Lattice Higgs Model?, Phys. Lett. B 212, 472 (1988); Scaling Laws and Triviality Bounds in the Lattice $\phi^{4}$ Theory. 1. One Component Model in the Symmetric Phase, Nucl. Phys. B 290, 25 (1987); Scaling Laws and Triviality Bounds in the Lattice $\phi^{4}$ Theory. 2. One Component Model in the Phase with Spontaneous Symmetry Breaking, Nucl. Phys. B 295, 65 (1988).

[97] C. B. Lang, On the Continuum Limit of $D=4$ Lattice $\phi^{4}$ Theory, Nucl. Phys. B 265, 630 (1986).

[98] D. J. E. Callaway, Triviality Pursuit: Can Elementary Scalar Particles Exist?, Phys. Rept. 167, 241 (1988).

[99] S. D. Bass, Emergence in Particle Physics, Acta Phys. Polon. B 48, 1903 (2017).

[100] F. Jegerlehner, The Vector Boson and Graviton Propagators in the Presence of Multipole Forces, Helv. Phys. Acta 51, 783 (1978).

[101] M. J. G. Veltman, Perturbation theory of massive Yang-Mills fields, Nucl. Phys. B 7, 637 (1968).

[102] C. H. Llewellyn Smith, High-Energy Behavior and Gauge Symmetry, Phys. Lett. B 46, 233 (1973). 
[103] J. S. Bell, High-energy Behavior of Tree Diagrams in Gauge Theories, Nucl. Phys. B 60, 427 (1973).

[104] J. M. Cornwall, D. N. Levin, G. Tiktopoulos, Uniqueness of spontaneously broken gauge theories, Phys. Rev. Lett. 30, 1268 (1973) [Erratum-ibid. 31, 572 (1973)]; Derivation of Gauge Invariance from High-Energy Unitarity Bounds on the $S$-Matrix, Phys. Rev. D 10, 1145 (1974) [Erratum-ibid. D 11, 972 (1975)].

[105] F. Jegerlehner, Search for anomalous gauge boson couplings, Nucl. Phys. Proc. Suppl. 37B, 129 (1994).

[106] F. Jegerlehner, The 'Ether world' and elementary particles," in: Theory of Elementary Particles. Eds. H. Dorn, D. Lüst, G. Weigt, Wiley-VCH, Berlin, 1998, p. 386 [hep-th/9803021].

[107] F. Jegerlehner, The hierarchy problem of the electroweak Standard Model revisited, arXiv:1305.6652 [hep-ph]; The hierarchy problem and the cosmological constant problem in the Standard Model, arXiv:1503.00809 [hep-ph].

[108] M. Lüscher, Chiral gauge theories revisited, Subnucl. Ser. 38, 41 (2002); Lattice regularization of chiral gauge theories to all orders of perturbation theory, JHEP 0006, 028 (2000).

[109] S. Elitzur, Impossibility of Spontaneously Breaking Local Symmetries, Phys. Rev. D 12, 3978 (1975).

[110] Y. Hamada, H. Kawai, K. Kawana, Natural solution to the naturalness problem: The universe does fine-tuning, PTEP 2015, 123B03 (2015).

[111] S. R. Coleman, E. J. Weinberg, Radiative Corrections as the Origin of Spontaneous Symmetry Breaking, Phys. Rev. D 7, 1888 (1973).

[112] V.L. Ginzburg, L.D. Landau, Zh. Eksp. Teor. Fiz. 20, 1064 (1950), English translation in: L. D. Landau, Collected papers (Oxford: Pergamon Press, 1965) p. 546; see also: E.M. Lifshitz, L. P. Pitaevskii, Statistical Physics: Theory of the Condensed State (Landau-Lifshitz Course of Theoretical Physics Vol. 9) (Pergamon, Oxford, 1980)

[113] L. N. Cooper, Bound Electron Pairs in a Degenerate Fermi Gas, Phys. Rev. 104, 1189 (1956);

J. Bardeen, L. N. Cooper, J. R. Schrieffer, Microscopic Theory of Superconductivity, Phys. Rev. 106, 162 (1957); Theory of Superconductivity, Phys. Rev. 108, 1175 (1957).

[114] L. P. Gor'kov, Microscopic Derivation of the Ginzburg-Landau Equations in the Theory of Superconductivity, Sov. Phys. JETP 9, 1364 (1959).

[115] A. A. Abrikosov, On the Magnetic properties of superconductors of the second group, Zh. Eksp. Teor. Fiz. 32, 1442 (1957) [Sov. Phys. JETP 51174 (1957)]. 
[116] I. Masina, Higgs boson and top quark masses as tests of electroweak vacuum stability, Phys. Rev. D 87, 053001 (2013).

[117] Y. Tang, Vacuum Stability in the Standard Model, Mod. Phys. Lett. A 28, 1330002 (2013).

[118] A. H. Hoang, The Top Mass: Interpretation and Theoretical Uncertainties, arXiv:1412.3649 [hep-ph].

[119] G. Degrassi, A. Sirlin, Gauge dependence of basic electroweak corrections of the standard model, Nucl. Phys. B 383, 73 (1992).

[120] Z. Y. Fang, G. Lopez Castro, J. L. Lucio, J. Pestieau, Effective $S U(2)_{L} \times U(1)$ theory and the Higgs boson mass, Mod. Phys. Lett. A 12, 1531 (1997).

[121] D. A. Kirzhnits, Weinberg model in the hot universe, JETP Lett. 15, 529 (1972) [Pisma Zh. Eksp. Teor. Fiz. 15, 745 (1972)].

[122] L. Dolan, R. Jackiw, Symmetry Behavior at Finite Temperature, Phys. Rev. D 9, 3320 (1974).

[123] S. Weinberg, Gauge and Global Symmetries at High Temperature, Phys. Rev. D 9, 3357 (1974).

[124] D. A. Kirzhnits, A. D. Linde, Symmetry Behavior in Gauge Theories, Annals Phys. 101, 195 (1976).

[125] M. Dine, R. G. Leigh, P. Y. Huet, A. D. Linde, D. A. Linde, Towards the theory of the electroweak phase transition, Phys. Rev. D 46, 550 (1992).

[126] S. Weinberg, Perturbative Calculations of Symmetry Breaking, Phys. Rev. D 7, 2887 (1973); Mass of the Higgs Boson, Phys. Rev. Lett. 36, 294 (1976).

[127] C. Ford, D. R. T. Jones, The Effective potential and the differential equations method for Feynman integrals, Phys. Lett. B 274, 409 (1992) Erratum: [Phys. Lett. B 285, 399 (1992)].

[128] C. Ford, I. Jack, D. R. T. Jones, The Standard model effective potential at two loops, Nucl. Phys. B 387, 373 (1992) Erratum: [Nucl. Phys. B 504, 551 (1997)]

[129] C. Ford, D. R. T. Jones, P. W. Stephenson, M. B. Einhorn, The Effective potential and the renormalization group, Nucl. Phys. B 395, 17 (1993).

[130] B. M. Kastening, Renormalization group improvement of the effective potential in massive $\phi^{4}$ theory, Phys. Lett. B 283, 287 (1992).

[131] S. P. Martin, Three-loop Standard Model effective potential at leading order in strong and top Yukawa couplings, Phys. Rev. D 89, no. 1, 013003 (2014).

[132] H. Nakano, Y. Yoshida, Improving the effective potential, multimass problem and modified mass dependent scheme, Phys. Rev. D 49, 5393 (1994). 
[133] C. P. Burgess, V. Di Clemente, J. R. Espinosa, Effective operators and vacuum instability as heralds of new physics, JHEP 0201, 041 (2002).

[134] N. Straumann, The Mystery of the cosmic vacuum energy density and the accelerated expansion of the universe, Eur. J. Phys. 20, 419 (1999).

[135] G. E. Volovik, Vacuum energy: Quantum hydrodynamics versus quantum gravity, JETP Lett. 82, 319 (2005) [Pisma Zh. Eksp. Teor. Fiz. 82, 358 (2005)].

[136] J. Solà, Cosmological constant and vacuum energy: old and new ideas, J. Phys. Conf. Ser. 453012015 (2013) [arXiv:1306.1527 [gr-qc]].

[137] D. H. Weinberg, M. White, Dark energy, review 27. in M. Tanabashi et al. (Particle Data Group), Review of Particle Physics, Phys. Rev. D 98, 010001 (2018).

[138] N. Cabibbo, Unitary Symmetry and Leptonic Decays, Phys. Rev. Lett. 10, 531 (1963);

M. Kobayashi, K. Maskawa, CP Violation in the Renormalizable Theory of Weak Interaction, Prog. Theor. Phys. 49, 652 (1973).

[139] S. Weinberg, Baryon and Lepton Nonconserving Processes, Phys. Rev. Lett. 43, 1566 (1979).

[140] W. Buchmüller, D. Wyler, Effective Lagrangian Analysis of New Interactions and Flavor Conservation, Nucl. Phys. B 268, 621 (1986).

[141] B. Grzadkowski, M. Iskrzynski, M. Misiak, J. Rosiek, Dimension-Six Terms in the Standard Model Lagrangian, JHEP 1010, 085 (2010).

[142] P. A. R. Ade et al. [Planck Collaboration], Planck 2013 results. XXII. Constraints on inflation, Astron. Astrophys. 571, A22 (2014).

[143] D. H. Lyth, What would we learn by detecting a gravitational wave signal in the cosmic microwave background anisotropy?, Phys. Rev. Lett. 78, 1861 (1997).

[144] H. Aoki, S. Iso, Revisiting the Naturalness Problem - Who is afraid of quadratic divergences? -, Phys. Rev. D 86, 013001 (2012).

[145] M. Blanke, G. F. Giudice, P. Paradisi, G. Perez, J. Zupan, Flavoured Naturalness, JHEP 1306, 022 (2013).

[146] G. M. Tavares, M. Schmaltz, W. Skiba, Higgs mass naturalness and scale invariance in the UV, Phys. Rev. D 89, 015009 (2014).

[147] I. Masina, M. Quiros, On the Veltman Condition, the Hierarchy Problem and High-Scale Supersymmetry, Phys. Rev. D 88, 093003 (2013).

[148] L. Bian, Renormalization group equation, the naturalness problem, and the understanding of the Higgs mass term, Phys. Rev. D 88, 056022 (2013). 
[149] F. Jegerlehner, The SM as a low energy effective theory and the role of the Higgs in the early universe, Lectures at IFJ-PAN, Krakow, October 14-27, 2014, http://www-com.physik.hu-berlin.de/ fjeger/SMcosmology.html.

[150] M. Veltman, The Importance of radiative corrections, in Radiative corrections: Results and Perspectives. Proceedings, NATO Advanced Research Workshop, Brighton, UK, July 10-14, 1989, eds. N. Dombey and F. Boudjema, NATO Sci. Ser. B 233, 1 (1990). See also:

C. Q. Geng, R. E. Marshak, Uniqueness of Quark and Lepton Representations in the Standard Model From the Anomalies Viewpoint, Phys. Rev. D 39 (1989) 693;

K. S. Babu, R. N. Mohapatra, Is There a Connection Between Quantization of Electric Charge and a Majorana Neutrino?, Phys. Rev. Lett. 63 (1989) 938;

J. A. Minahan, P. Ramond, R. C. Warner, A Comment on Anomaly Cancellation in the Standard Model, Phys. Rev. D 41 (1990) 715;

S. Rudaz, Electric charge quantization in the standard model, Phys. Rev. D 41 (1990) 2619.

[151] F. Jegerlehner, Renormalizing the standard model, in Testing the Standard Model - TASI-90 proceedings. Eds. M. Cvetic, P. Langacker. Teaneck, N.J., World Scientific, 1991, 916p.

[152] P. Corning,The Re-Emergence of Emergence: A Venerable Concept in Search of a Theory, (2002). Complexity, 7(6): 18-30. 